\title{
Arylboronic Acid-Catalyzed C-Alkylation and Allylation Reactions Using Benzylic Alcohols
}

\author{
Susana Estopiñá-Durán, ${ }^{\dagger, \dagger}$ Euan B. Mclean,${ }^{\dagger}$ Liam J. Donnelly, ${ }^{\dagger}$ Bryony M. Hockin, ${ }^{\dagger}$ \\ James E. Taylor*, \\ ${ }^{\dagger}$ EaStCHEM, School of Chemistry, University of St Andrews, North Haugh, St Andrews, KY16 9ST, U.K. \\ *Department of Chemistry, University of Bath, Claverton Down, Bath, Somerset, BA2 7AY, U.K.
}

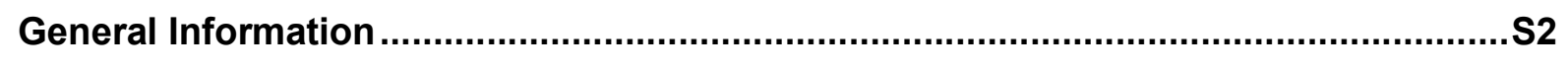

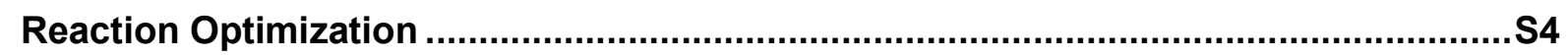

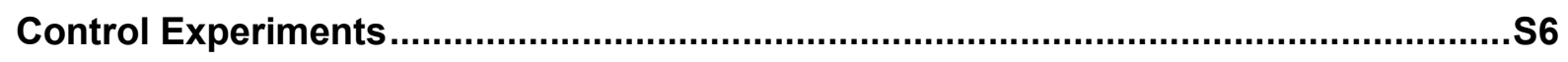

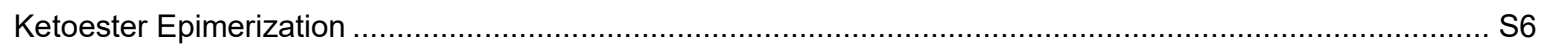

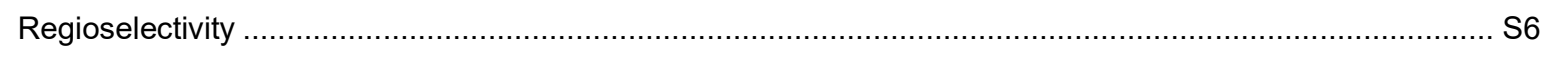

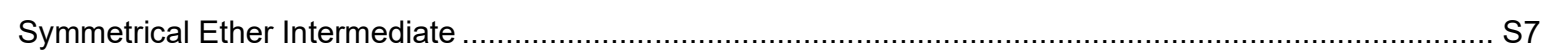

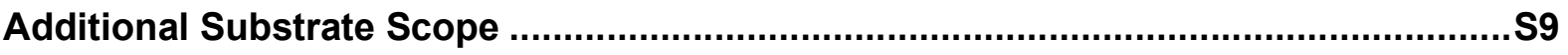

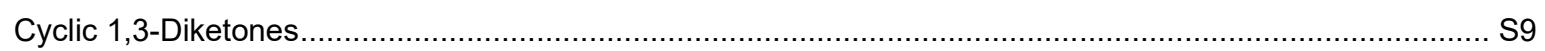

1,3-Ketoesters

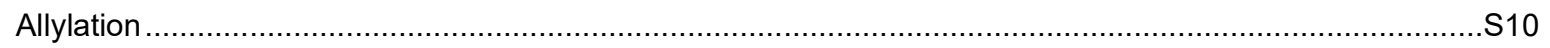

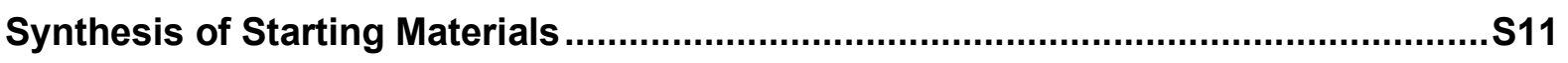

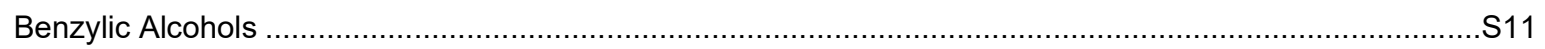

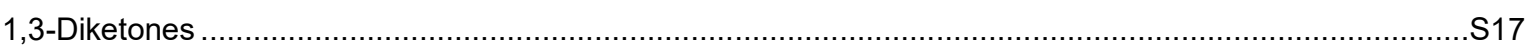

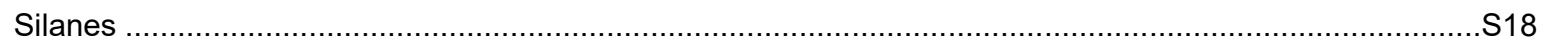

Dehydrative Alkylation of 1,3-Diketone Derivatives ............................................. 19

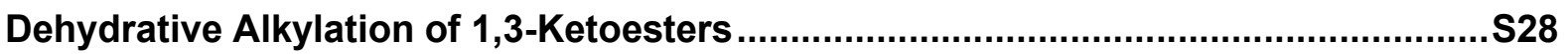

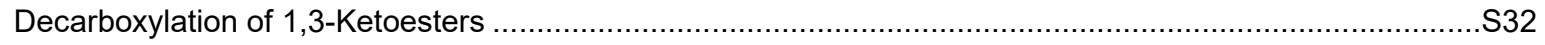

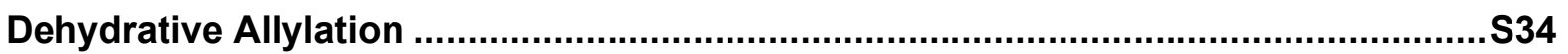

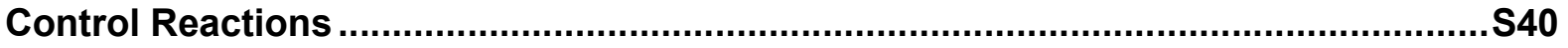

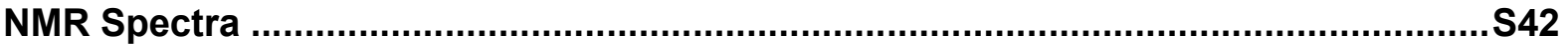

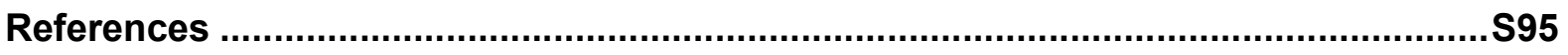




\section{General Information}

Reactions involving moisture sensitive reagents were carried out in flame-dried glassware under an inert atmosphere $\left(\mathrm{N}_{2}\right)$ using standard vacuum line techniques. Anhydrous solvents $\left(\mathrm{Et}_{2} \mathrm{O}, \mathrm{CH}_{2} \mathrm{Cl}_{2}\right.$, and THF) were obtained after passing through an alumina column (Mbraun SPS-800). Petrol is defined as petroleum ether $40-60{ }^{\circ} \mathrm{C}$. All other solvents and commercial reagents were used as received without further purification unless otherwise stated.

Room temperature (rt) refers to $20-25^{\circ} \mathrm{C}$. Temperatures of $0{ }^{\circ} \mathrm{C}$ were obtained using an ice/water bath. Reaction involving heating were performed using DrySyn blocks and a contact thermocouple.

Analytical thin layer chromatography was performed on pre-coated aluminium plates (Kieselgel 60 F254 silica) and visualisation was achieved using ultraviolet light (254 nm) and/or staining with either aqueous $\mathrm{KMnO}_{4}$ solution or ethanolic phosphomolybdic acid followed by heating. Manual column chromatography was performed in glass columns fitted with porosity 3 sintered discs over Kieselgel 60 silica using the solvent system stated.

Melting points were recorded on either an Electrothermal 9100 melting point apparatus or an Stanford Research Systems OptiMelt automated capillary melting point apparatus in open capillary tubes, (dec) refers to decomposition.

Infrared spectra were recorded on either a Shimadzu IRAffinity-1 Fourier transform IR spectrophotometer fitted with a Specac Quest ATR accessory (diamond puck) or on a PerkinElmer PerkinElmer Spectrum 100 ATR-FTIR spectrometer. Spectra were recorded of either thin films or solids, with characteristic absorption wavenumbers $\left(v_{\max }\right)$ reported in $\mathrm{cm}^{-1}$.

${ }^{1} \mathrm{H},{ }^{13} \mathrm{C}\left\{{ }^{1} \mathrm{H}\right\}$, and ${ }^{19} \mathrm{~F}\left\{{ }^{1} \mathrm{H}\right\}$ NMR spectra were acquired on either a Bruker AV300 $\left({ }^{1} \mathrm{H} 300 \mathrm{MHz}\right.$; $\left.{ }^{13} \mathrm{C}\left\{{ }^{1} \mathrm{H}\right\} 75 \mathrm{MHz} ;{ }^{19} \mathrm{~F}\left\{{ }^{1} \mathrm{H}\right\} 282 \mathrm{MHz}\right)$, a Bruker AV400 $\left({ }^{1} \mathrm{H} 400 \mathrm{MHz} ;{ }^{13} \mathrm{C}\left\{{ }^{1} \mathrm{H}\right\} 101 \mathrm{MHz}\right.$; $\left.{ }^{19} \mathrm{~F}\left\{{ }^{1} \mathrm{H}\right\} 376 \mathrm{MHz}\right)$, a Bruker AVII $400\left({ }^{1} \mathrm{H} 400 \mathrm{MHz} ;{ }^{13} \mathrm{C}\left\{{ }^{1} \mathrm{H}\right\} 101 \mathrm{MHz} ;{ }^{19} \mathrm{~F}\left\{{ }^{1} \mathrm{H}\right\} 376 \mathrm{MHz}\right)$, a Bruker Neo $400\left({ }^{1} \mathrm{H} 400 \mathrm{MHz} ;{ }^{13} \mathrm{C}\left\{{ }^{1} \mathrm{H}\right\} 101 \mathrm{MHz} ;{ }^{19} \mathrm{~F}\left\{{ }^{1} \mathrm{H}\right\} 376 \mathrm{MHz}\right)$, a Bruker AVIII-HD $500\left({ }^{1} \mathrm{H} 500 \mathrm{MHz},{ }^{13} \mathrm{C}\left\{{ }^{1} \mathrm{H}\right\} 126 \mathrm{MHz},{ }^{19} \mathrm{~F}\left\{{ }^{1} \mathrm{H}\right\} 470 \mathrm{MHz},{ }^{11} \mathrm{~B} 160 \mathrm{MHz}\right)$, a Bruker AVIII 500 $\left({ }^{1} \mathrm{H} 500 \mathrm{MHz},{ }^{13} \mathrm{C}\left\{{ }^{1} \mathrm{H}\right\} 126 \mathrm{MHz},{ }^{19} \mathrm{~F}\left\{{ }^{1} \mathrm{H}\right\} 470 \mathrm{MHz},{ }^{11} \mathrm{~B} 160 \mathrm{MHz}\right)$, or an Agilent ProPulse $500\left({ }^{1} \mathrm{H} 500 \mathrm{MHz},{ }^{13} \mathrm{C}\left\{{ }^{1} \mathrm{H}\right\} 126 \mathrm{MHz},{ }^{19} \mathrm{~F}\left\{{ }^{1} \mathrm{H}\right\} 470 \mathrm{MHz}\right)$ in the deuterated solvent stated. All chemical shifts are quoted in parts per million (ppm) relative to the residual solvent peak. All coupling constants, $J$, are quoted in Hz. Multiplicities are indicated as s (singlet), d (doublet), $\mathrm{t}$ (triplet), q (quartet), m (multiplet), and multiples thereof. The abbreviation Ar denotes aromatic and app denotes apparent. NMR peak assignments were confirmed using $2 \mathrm{D}{ }^{1} \mathrm{H}$ 
correlated spectroscopy (COSY), 2D ${ }^{1} \mathrm{H}^{-13} \mathrm{C}$ heteronuclear multiple-bond correlation spectroscopy (HMBC), and 2D ${ }^{1} \mathrm{H}^{-13} \mathrm{C}$ heteronuclear single quantum coherence (HSQC) where necessary.

Mass spectrometry $(\mathrm{m} / \mathrm{z})$ data were acquired by either electrospray ionisation (ESI), chemical ionisation (CI), electron impact (EI), atmospheric solids analysis probe (ASAP), atmospheric pressure chemical ionization (APCI) or nanospray ionisation (NSI) at either the University of St Andrews Mass Spectrometry Facility ([A] quoted), the EPSRC UK National Mass Spectrometry Facility at Swansea University ([A $]^{+}$or $[\mathrm{A}]^{-}$quoted), or at the University of Bath $\left([\mathrm{A}]^{+}\right.$or $[\mathrm{A}]^{-}$quoted) using either a TOF or QTof mass analyzer. 


\section{Reaction Optimization}

\section{Table S1. Catalyst and Ligand Screening ${ }^{a}$}

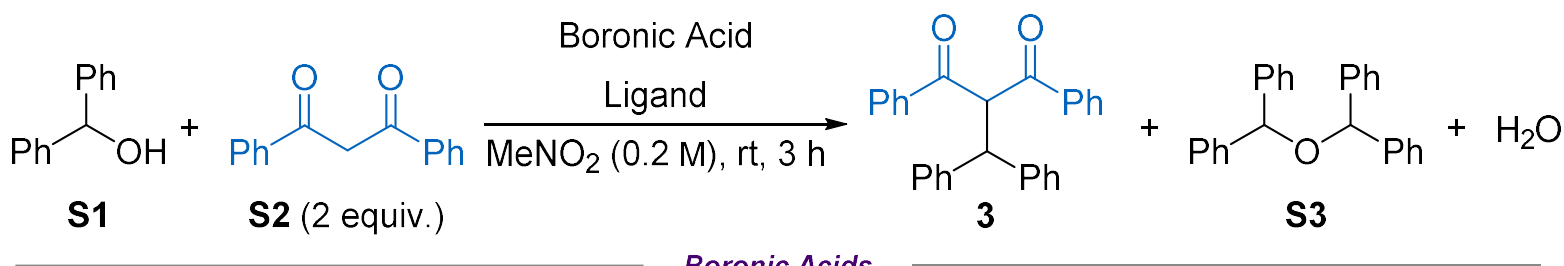<smiles>Oc1c(F)c(F)c(F)c(F)c1F</smiles>

1<smiles>Oc1cc(F)c(F)c(Br)c1</smiles>

S4<smiles>FC(F)(F)c1cc(Br)cc(C(F)(F)F)c1</smiles>

S5<smiles>O=[N+]([O-])c1ccccc1Br</smiles>

S6<smiles>COc1ccccc1C(=O)O</smiles>

S7<smiles>O=C(O)C(O)c1ccccc1</smiles>

2<smiles>O=C(O)C(O)C(O)C(=O)O</smiles>

S8<smiles>O=C(O)CC(=O)O</smiles>

S9<smiles>O=C(O)c1ccccc1C(=O)O</smiles>

$\mathrm{S} 10$

$\begin{array}{ccccc}\text { Entry } & \text { Boronic acid (mol\%) } & \text { Ligand (mol\%) } & \text { Conversion }^{b} & \mathbf{3 : S 3}^{b} \\ 1 & - & - & 0 \% & \text { N/A } \\ 2 & - & \mathbf{2}(10) & 5 \% & 100: 0 \\ 3 & \mathbf{1}(5) & \mathbf{2}(10) & 100 \% & 99: 1 \\ 4 & \mathbf{S 4}(5) & \mathbf{2}(10) & 77 \% & 91: 9 \\ 5 & \mathbf{S 5}(5) & \mathbf{2}(10) & 83 \% & 90: 10 \\ 6 & \mathbf{S 6}(5) & \mathbf{2}(10) & 65 \% & 91: 9 \\ 7 & \mathbf{S 7}(5) & \mathbf{2}(10) & 98 \% & 94: 6 \\ 8 & \mathbf{1}(5) & - & <1 \% & \text { N/A } \\ 9 & \mathbf{1}(5) & \mathbf{S 8}(10) & <5 \% & \text { N/A } \\ 10 & \mathbf{1}(5) & \mathbf{S 9}(10) & <5 \% & \text { N/A } \\ 11 & \mathbf{1}(5) & \mathbf{S 1 0}(10) & <5 \% & \text { N/A } \\ 12 & \mathbf{1}(5) & \mathbf{2}(2.5) & 56 \% & 89: 11 \\ 13 & \mathbf{1}(5) & \mathbf{2}(5) & 75 \% & 90: 10 \\ 14 & \mathbf{1}(2) & \mathbf{2}(4) & 60 \% & 88: 12\end{array}$

${ }^{a}$ Reactions performed on $0.2 \mathrm{mmol}$ scale. ${ }^{b}$ Determined by ${ }^{1} \mathrm{H}$ NMR analysis. 
Table S2. Solvent, Concentration and Equivalents Screening ${ }^{a}$

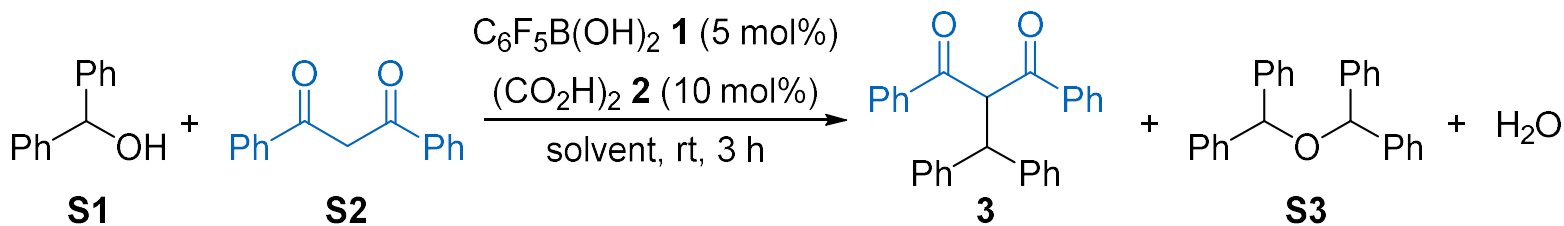

S1

S2

3

S3

$\begin{array}{ccccc}\text { Entry } & \text { S2 (equiv.) } & \text { Solvent (M) } & \text { Conversion }^{b} & \mathbf{3 : S 3} \\ 1 & 2 & \mathrm{MeNO}_{2}(0.2) & 100 \% & 99: 1 \\ 2 & 2 & \mathrm{HFIP}^{c}(0.2) & 100 \% & >99: 1 \\ 3 & 2 & \mathrm{MeCN}(0.2) & 34 \% & 62: 38 \\ 4 & 2 & \mathrm{PhMe}^{b}(0.2) & <5 \% & \mathrm{~N} / \mathrm{A} \\ 5 & 2 & \mathrm{CH}_{2} \mathrm{Cl}_{2}(0.2) & 7 \% & 57: 43 \\ 6 & 2 & \mathrm{MeNO}_{2}(0.5) & 99 \% & 95: 5 \\ 7 & 2 & \mathrm{MeNO}_{2}(0.1) & 100 \% & >99: 1 \\ 8 & 2 & \mathrm{MeNO}_{2}(0.05) & 100 \%(76 \%)^{d} & >99: 1 \\ 9 & 1.5 & \mathrm{MeNO}_{2}(0.05) & 100 \% & >99: 1 \\ 10 & 1.2 & \mathrm{MeNO}_{2}(0.05) & 100 \% & >99: 1 \\ 11 & 1 & \mathrm{MeNO}_{2}(0.05) & 100 \% & >99: 1\end{array}$

${ }^{a}$ Entries 1-8 performed on $0.1 \mathrm{mmol}$ scale, entries $9-11$ performed on $0.2 \mathrm{mmol}$ scale. ${ }^{b}$ Determined by ${ }^{1} \mathrm{H}$ NMR analysis. ${ }^{c} 1,1,1,3,3,3$-Hexafluoro-2-propanol. ${ }^{d}$ Isolated yield. 


\section{Control Experiments}

\section{Ketoester Epimerization}

Reacting an isolated sample of 21 (63:37 dr) under the standard conditions for $4 \mathrm{~h}$ at rt resulted in a change in the diastereomeric ratio (to $53: 47 \mathrm{dr}$ ), showing that the catalyst may promote epimerization of the products, leading to formation of a thermodynamic mixture of diastereoisomers.

Scheme S1. Ketoester Epimerization
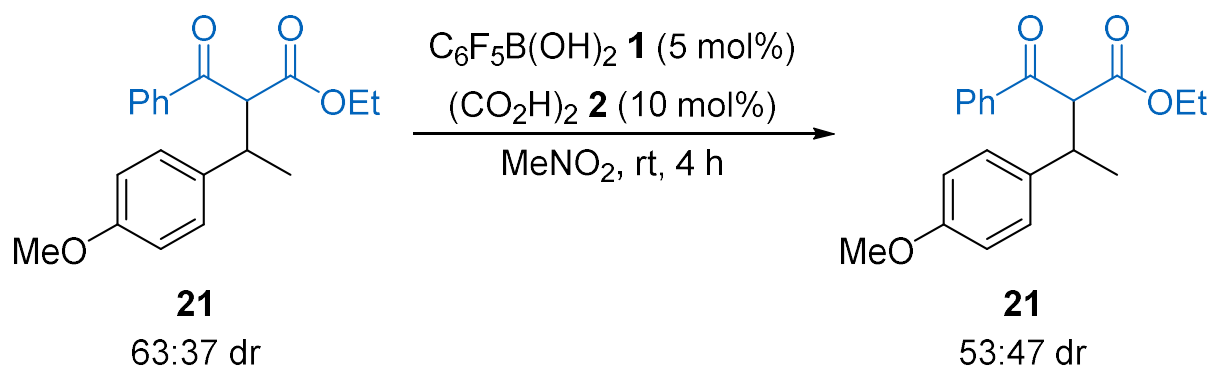

\section{Regioselectivity}

Reacting dibenzoylmethane (2 equiv.) with either $\alpha$-vinylbenzyl alcohol S11 (Scheme S2a) or isomeric cinnamyl alcohol S12 (Scheme S2b) under the standard conditions led to a compariable yield and mixture of regioisomeric products S13 and S14 in both cases, with the linear $C$-alkylation product favoured.

\section{Scheme S2. C-Alkylation Regioselectivity}

a)

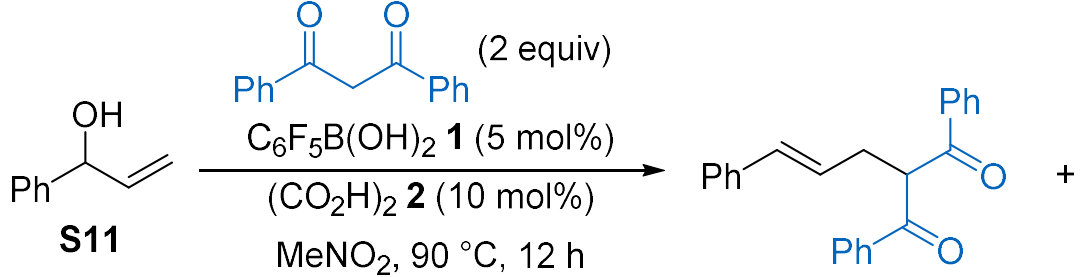

S13<smiles>C=CC(c1ccccc1)C(C(=O)c1ccccc1)C(=O)c1ccccc1</smiles>

S14

85:15 S13:S14

b)

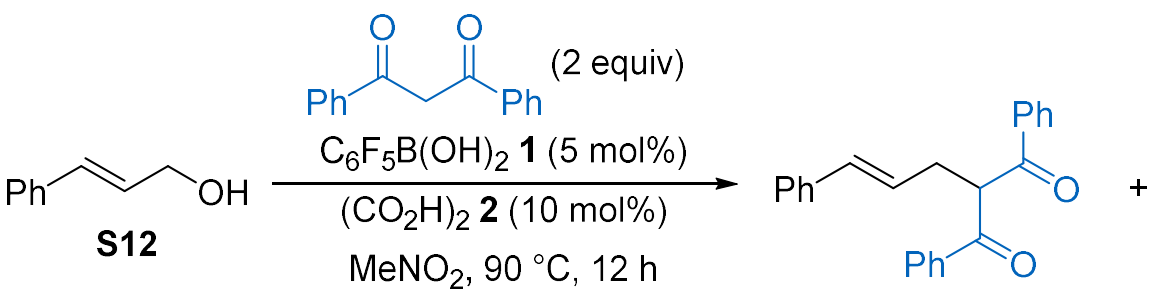

S13<smiles>C=CC(c1ccccc1)C(C(=O)P)C(=O)c1ccccc1</smiles>

S14 
Reacting allyltrimethylsilane (2 equiv.) with either $\alpha$-vinylbenzyl alcohol 39 (Scheme S3a) or isomeric cinnamyl alcohol 40 (Scheme $\mathrm{S} 3 \mathrm{~b}$ ) under the standard conditions gave complete selectivity for linear product $\mathbf{S 1 5}$ in both cases in compariable yield. These results support the formation of a common cation intermediate leading to the same product distributions from isomeric starting materials.

\section{Scheme S3. Allylation Regioselectivity}

a)

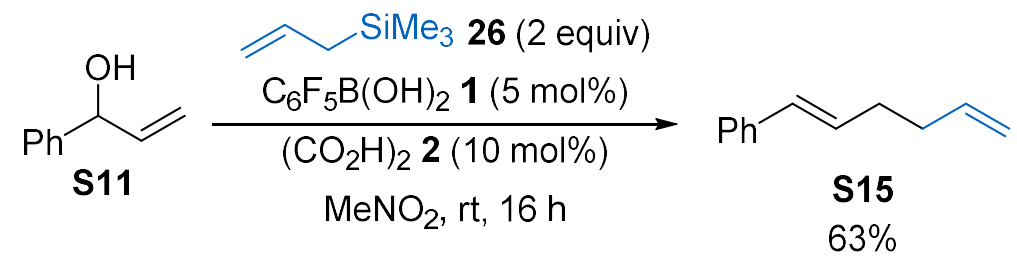

b)

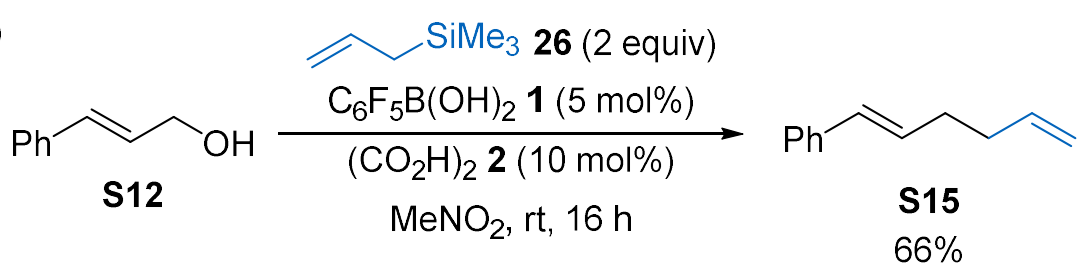

\section{Symmetrical Ether Intermediate}

Hall and co-workers have shown that symmetric ether formation was kinetically favored for a 2,3,4,5-tetrafluorophenylboronic acid (10 mol\%) and perfluoropinacol (10 mol\%) catalyzed Friedel-Crafts alkylation reaction using benzylic alcohols, with the ether subsequently being converted into the desired product. ${ }^{1}$ Reaction monitoring showed that the symmetrical ether is likely to be an intermediate and a control experiment showed the ether was a competent starting material. To investigate this possibility for the reaction of 1,3-diketones, ether $\mathbf{S 3}$ was reacted under the standard reaction conditions for $3 \mathrm{~h}$ at $\mathrm{rt}$ (Scheme S4). Analysis of the crude reaction mixture with ${ }^{1} \mathrm{H}$ NMR showed 58\% conversion of ether $\mathbf{S 3}$ into mainly $C$-alkylation product 3, with a small amount of benzyhydrol S1 also observed.

\section{Scheme S4. Symmetrical Ether as Starting Material in $C$-Alkylation}

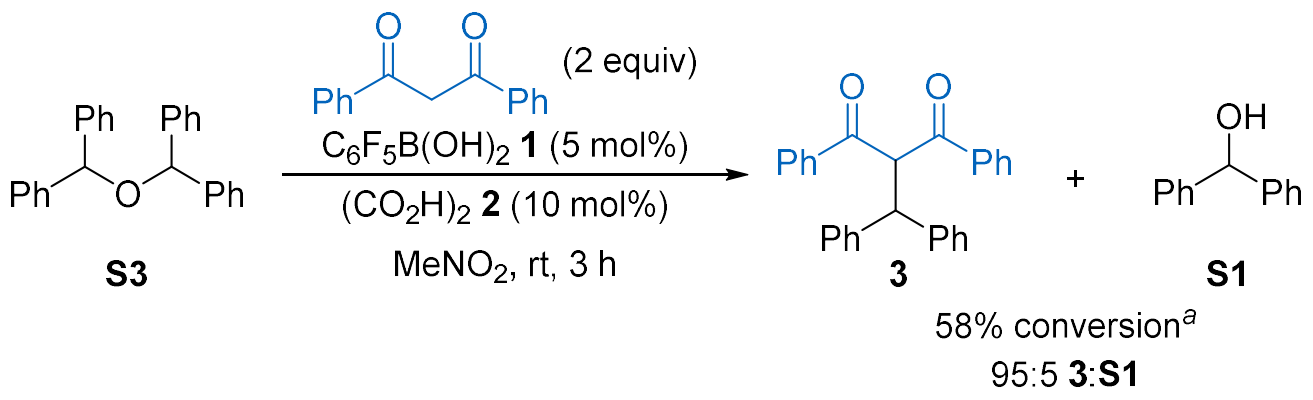


The same experimenmt was performed using ether $\mathbf{S 3}$ and allyltrimethylsilane $\mathbf{2 6}$ (2 equiv.) under the standard reaction conditions in $\mathrm{MeNO}_{2}$ at $90{ }^{\circ} \mathrm{C}$ for $16 \mathrm{~h}(\mathrm{Scheme} \mathrm{S} 1)$. Again, ${ }^{1} \mathrm{H}$ NMR analysis showed ether S3 was a competent precursor, giving 74\% conversion into product 27 (Scheme S5a). However, an analogous experiment using symmetric ether S16 (1:1 dr) showed no reaction by ${ }^{1} \mathrm{H}$ NMR spectroscopy after $16 \mathrm{~h}$ (Scheme S5b), suggesting that the role of symmetric ethers as potential intermediates is substrate specific.

\section{Scheme S5. Symmetrical Ethers as Starting Material in Allylation}

a)

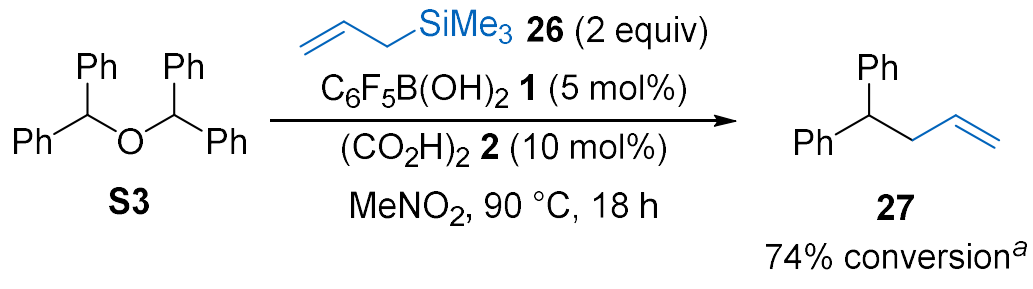

b)

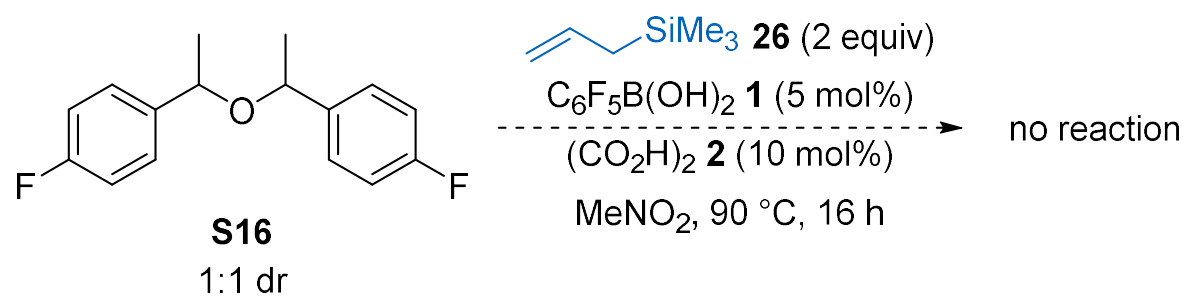




\section{Additional Substrate Scope}

\section{Cyclic 1,3-Diketones}

The reaction of benzhydrol with 1,3-cyclohexanedione gave selective $O$-alkylation under the standard reaction conditions, forming $\beta$-keto enol ether $\mathbf{S 1 7}$ in $73 \%$ yield (Scheme S6). This process was specific to benzyhdrol, with a range of other secondary benzylic alcohols returning only starting materials under the same conditions.

Scheme S6. Reaction with 1,3-Cyclohexanedione<smiles>O=C1C=C(OC(c2ccccc2)c2ccccc2)CCC1</smiles>

Unsuccessful substrates that were evaluated under the standard reaction conditions for the dehydrative alkylation of 1,3-diketones in Scheme S7 below.

\section{Scheme S7. Addition Substrate Evaluation with 1,3-Diketones}
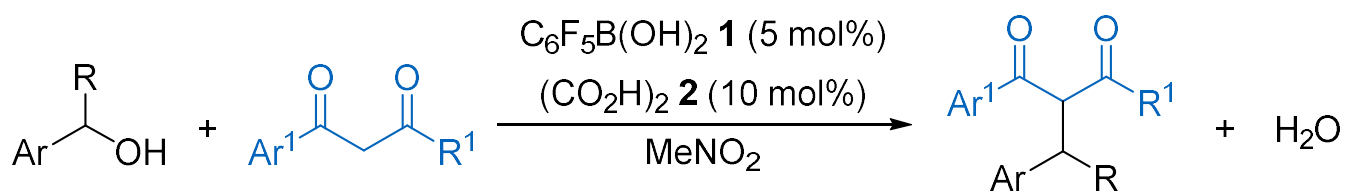

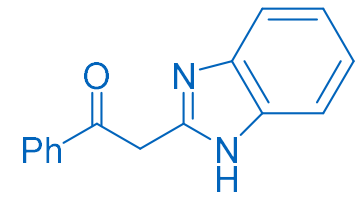

no reaction

Unsuccessful Nucleophiles<smiles>O=C(Cc1ccccc1)c1ccccc1</smiles>

no reaction<smiles>N#CCC(=O)c1ccccc1</smiles>

no reaction

Unsuccessful Substrates<smiles>OC[PbH2]</smiles>

ether formation<smiles>CC(C)(C)[Ge](O)C(O)c1ccccc1</smiles>

no reaction<smiles>CC(C)(O)P</smiles>

no reaction<smiles>C[As](C)CC(O)c1ccccc1</smiles>

complex mixture 


\section{1,3-Ketoesters}

Unsuccessful substrates that were evaluated under the standard reaction conditions for the dehydrative alkylation of ethyl benzoylacetate are show in Scheme S8 below.

\section{Scheme S8. Addition Substrate Evaluation with 1,3-Ketoesters}

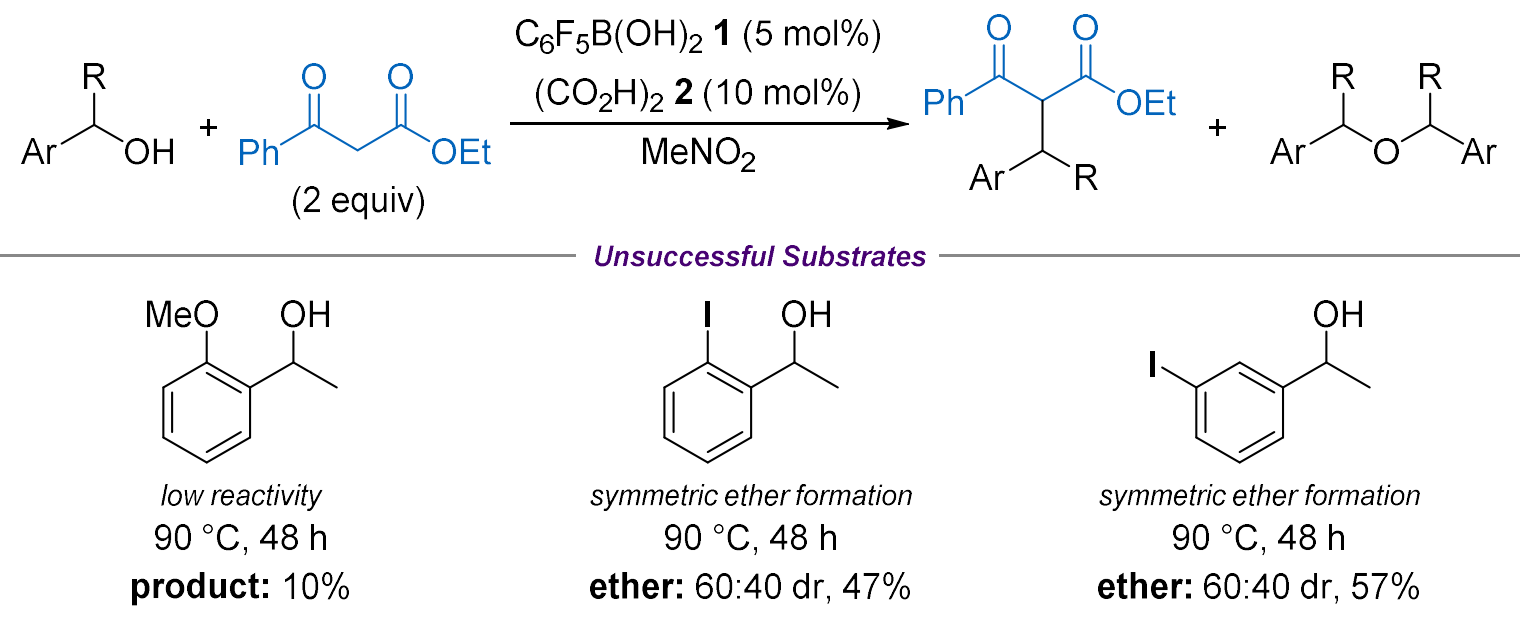<smiles>C#CC(O)c1ccc(OC)cc1</smiles><smiles>CC(C)(C(=O)O)C(O)c1ccccc1</smiles><smiles>CSCC(O)c1ccccc1</smiles>

\section{Allylation}

Unsuccessful substrates that were evaluated under the standard reaction conditions for the allylation of benzylic alcohols are show in Scheme S9 below.

\section{Scheme S9. Addition Substrate Evaluation for the Allylation of Benzylic Alcohols}
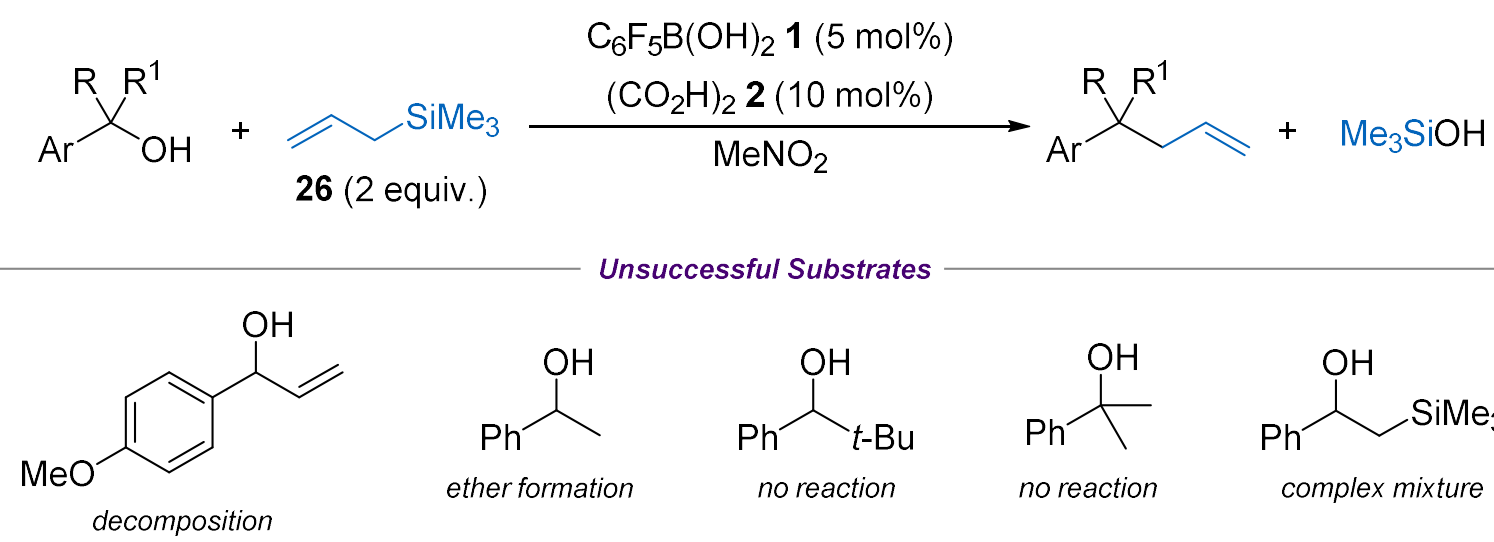<smiles>CC(C)(C)C(O)c1ccccc1</smiles><smiles>CC(C)(O)c1ccccc1</smiles><smiles>CSCC(O)c1ccccc1</smiles> 


\section{Synthesis of Starting Materials}

\section{Benzylic Alcohols}

All other benzylic aclohols were used as received from commercial sources.

General Procedure A: Grignard addition

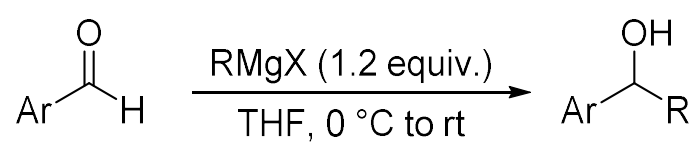

The required Grignard reagent (1.2 equiv.) was added dropwise to a solution of the required aldehyde (1.0 equiv.) in anhydrous THF $(0.35 \mathrm{M})$ at $0{ }^{\circ} \mathrm{C}$ under an atmosphere of $\mathrm{N}_{2}$. The mixture was stirred at $0{ }^{\circ} \mathrm{C}$ for $30 \mathrm{~min}$ and then at $\mathrm{rt}$ for $6 \mathrm{~h}$. The reaction was quenched with aq. $\mathrm{NH}_{4} \mathrm{Cl}$ and the organic layer was separated. The aqueous layer was extracted with EtOAc $(3 \times 20 \mathrm{~mL})$. The combined organic layers were washed with brine $(3 \times 10 \mathrm{~mL})$, dried over $\mathrm{MgSO}_{4}$, filtered, and concentrated under reduced pressure. The residual oil was either purified by silica-gel column chromatography (petrol/EtOAc) or used without further purification.

General Procedure B: Reduction

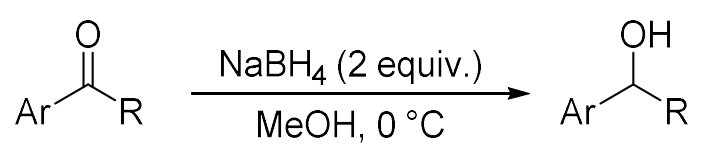

Sodium borohydride (2.0 equiv.) was added to a solution of the required aldehyde (1.0 equiv.) dissolved in methanol $(0.4 \mathrm{M})$ at $0{ }^{\circ} \mathrm{C}$. The mixture was stirred at $0{ }^{\circ} \mathrm{C}$ until complete by TLC analysis. The mixture was concentrated under reduced pressure before being diluted with $\mathrm{CH}_{2} \mathrm{Cl}_{2}$, washed with water, dried over $\mathrm{Na}_{2} \mathrm{SO}_{4}$, filtered, and concentrated under reduced pressure. to afford the product, which was used without further purification.

\section{1-Phenylethan-1-ol S18}

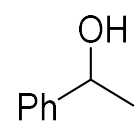

Following General Procedure A, benzaldehyde (1.04 g, $9.8 \mathrm{mmol})$ and $\mathrm{MeMgBr}(3.0 \mathrm{M}$ in THF, $3.93 \mathrm{~mL}, 11.8 \mathrm{mmol})$ were reacted in THF $(24 \mathrm{~mL})$. The reaction did not require further purification to give title compound $\mathbf{S 1 8}(1.20 \mathrm{~g}, 99 \%)$ as an orange oil, with spectroscopic data in accordance with the literature. ${ }^{2}{ }^{1} \mathrm{H}$ NMR $\left(400 \mathrm{MHz}, \mathrm{CDCl}_{3}\right) \delta_{\mathrm{H}}: 7.46-7.35(\mathrm{~m}, 3 \mathrm{H}$, $\operatorname{ArC}(3,4,5) H), 7.34-7.25(\mathrm{~m}, 2 \mathrm{H}, \operatorname{ArC}(2,6) H), 4.90(\mathrm{q}, J=6.5 \mathrm{~Hz}, 1 \mathrm{H}, \mathrm{OCH}), 2.04(\mathrm{~s}, 1 \mathrm{H}, \mathrm{OH})$, $1.50\left(\mathrm{~d}, J=6.5 \mathrm{~Hz}, 3 \mathrm{H}, \mathrm{CHCH}_{3}\right)$. 


\section{1-(4-Methoxyphenyl)ethan-1-ol S19}<smiles>COc1ccc(C(C)O)cc1</smiles>

Following General Procedure A, 4-methoxybenzaldehyde (1.33 g, $9.8 \mathrm{mmol})$ and $\mathrm{MeMgBr}$ (3.0 $\mathrm{M}$ in THF, $3.93 \mathrm{~mL}, 11.8 \mathrm{mmol})$ were reacted in THF $(24 \mathrm{~mL})$. The crude product was purified by silica-gel column chromatography (petrol/EtOAc, 9:1 to $7: 3, \mathrm{R}_{f} 0.39(8: 2)$ ) to give title compound $\mathbf{S 1 9}(0.517 \mathrm{~g}, 35 \%)$ as colourless oil, with spectroscopic data in accordance with the literature. ${ }^{2} \mathrm{H}_{\mathrm{NMR}}\left(400 \mathrm{MHz}, \mathrm{CDCl}_{3}\right) \delta_{\mathrm{H}}$ : 7.35-7.22 (m, 2H, $\left.\mathrm{ArC}(2,6) H\right), 6.93-6.82$ $(\mathrm{m}, 2 \mathrm{H}, \operatorname{ArC}(3,5) H), 4.85(\mathrm{q}, J=6.4 \mathrm{~Hz}, 1 \mathrm{H}, \mathrm{PhCHOH}), 3.80\left(\mathrm{~s}, 3 \mathrm{H}, \mathrm{OCH}_{3}\right), 1.83(\mathrm{~s}, 1 \mathrm{H}, \mathrm{OH})$, $1.47\left(\mathrm{~d}, J=6.4 \mathrm{~Hz}, 3 \mathrm{H}, \mathrm{CHCH}_{3}\right)$.

\section{1-(2-Methoxyphenyl)ethan-1-ol S20}<smiles>COc1ccccc1C(C)O</smiles>

Following General Procedure A, 2-methoxybenzaldehyde (0.50 g, $3.7 \mathrm{mmol})$ and $\mathrm{MeMgBr}$ (3.0 $\left.\mathrm{M}_{\text {in }} \mathrm{Et}_{2} \mathrm{O}, 1.47 \mathrm{~mL}, 4.41 \mathrm{mmol}\right)$ were reacted in $\mathrm{Et}_{2} \mathrm{O}(15 \mathrm{~mL})$. The reaction did not require further purification to give title compound S20 $(0.51 \mathrm{~g}, 89 \%)$ as an orange oil, with spectroscopic data in accordance with the literature. ${ }^{2}{ }^{1} \mathrm{H}$ NMR $\left(400 \mathrm{MHz}, \mathrm{CDCl}_{3}\right) \delta_{\mathrm{H}}: 7.40$ 7.34 (m, 1H, ArH), 7.32-7.25 (m, 1H, ArH), 7.04-6.95 (m, 1H, ArH), 6.95-6.87 (m, 1H, ArH), 5.17-5.07 (m, 1H, $\left.\mathrm{CHCH}_{3}\right), 3.90\left(\mathrm{~s}, 3 \mathrm{H}, \mathrm{OCH}_{3}\right), 2.66(\mathrm{~s}, 1 \mathrm{H}, \mathrm{OH}), 1.54(\mathrm{~d}, J=6.6 \mathrm{~Hz}, 3 \mathrm{H}$, $\left.\mathrm{CHCH}_{3}\right)$.

\section{1-(4-Fluorophenyl)ethan-1-ol, S21}<smiles>CC(O)c1ccc(F)cc1</smiles>

Following General Procedure B, 1-(4-fluorophenyl)ethan-1-one (2.0 mL, $16.5 \mathrm{mmol})$ and $\mathrm{NaBH}_{4}(1.25 \mathrm{~g}, 33 \mathrm{mmol})$ were reacted in $\mathrm{MeOH}(83 \mathrm{~mL})$. The crude product was purified by silica-gel column chromatography (petrol/EtOAc, 80:20 to 70/30, $\mathrm{R}_{f}$. 0.31 ) to give title compound S21 (1.94 g, 84\%) as a colourless oil, with spectroscopic data in accordance with the literature. ${ }^{2} \mathrm{H}$ NMR $\left(300 \mathrm{MHz}, \mathrm{CDCl}_{3}\right) \delta_{\mathrm{H}}: 7.41-7.28(\mathrm{~m}, 2 \mathrm{H}, \operatorname{Ar}(2,6) H), 7.10-6.97(\mathrm{~m}, 2 \mathrm{H}$, $\operatorname{ArC}(3,5) H), 4.89$ (q, $\left.J=6.4 \mathrm{~Hz}, 1 \mathrm{H}, \mathrm{CHCH}_{3}\right), 1.48$ (d, $\left.J=6.5 \mathrm{~Hz}, 3 \mathrm{H}, \mathrm{CHCH}_{3}\right)$. 


\section{1-(4-Bromophenyl)ethan-1-ol S22}<smiles>CC(O)c1ccc(Br)cc1</smiles>

Following General Procedure A, 4-bromobenzaldehyde (1.81 g, $9.8 \mathrm{mmol})$ and $\mathrm{MeMgBr}$ (3.0 $\mathrm{M}$ in THF, $3.93 \mathrm{~mL}, 11.8 \mathrm{mmol})$ were reacted in THF $(24 \mathrm{~mL})$. The reaction did not require further purification to give title compound S22 (1.95 g, 99\%) as an orange oil, with spectroscopic data in accordance with the literature. ${ }^{2}{ }^{1} \mathrm{H}$ NMR $\left(400 \mathrm{MHz}, \mathrm{CDCl}_{3}\right) \delta_{\mathrm{H}}: 7.52-$ 7.42 (m, 2H, ArH), 7.33-7.21 (m, 2H, ArH), 4.86 (q, $\left.J=6.5 \mathrm{~Hz}, 1 \mathrm{H}, \mathrm{CHCH}_{3}\right), 1.98(\mathrm{~s}, 1 \mathrm{H}$, $\mathrm{OH}), 1.53-1.45\left(\mathrm{~m}, 3 \mathrm{H}, \mathrm{CHCH}_{3}\right)$.

\section{1-(3-Bromophenyl)ethan-1-ol S23}<smiles>CC(O)c1cccc(Br)c1</smiles>

Following General Procedure A, 3-bromobenzaldehyde (1.81 g, $9.8 \mathrm{mmol})$ and $\mathrm{MeMgBr}(3.0$ $\mathrm{M}$ in THF, $3.93 \mathrm{~mL}, 11.8 \mathrm{mmol})$ were reacted in THF $(24 \mathrm{~mL})$. The reaction did not require further purification to give title compound S23 (1.92 g, 98\%) as an orange oil, with spectroscopic data in accordance with the literature. ${ }^{2}{ }^{1} \mathrm{H} \mathrm{NMR}\left(400 \mathrm{MHz}, \mathrm{CDCl}_{3}\right) \delta_{\mathrm{H}}: 7.57-$ 7.47 (m, 1H, ArH), 7.43-7.34 (m, 1H, ArH), 7.31-7.23 (m, 1H, ArH), 7.23-7.13 (m, 1H, ArH), $4.84\left(\mathrm{q}, J=6.5 \mathrm{~Hz}, 1 \mathrm{H}, \mathrm{CHCH}_{3}\right), 2.15(\mathrm{~s}, 1 \mathrm{H}, \mathrm{OH}), 1.46\left(\mathrm{~d}, J=6.5 \mathrm{~Hz}, 3 \mathrm{H}, \mathrm{CHCH}_{3}\right)$.

\section{1-(2-Bromophenyl)ethan-1-ol S24}<smiles>CC(O)c1ccccc1Br</smiles>

Following General Procedure A, 2-bromobenzaldehyde (1.81 g, $9.8 \mathrm{mmol})$ and $\mathrm{MeMgBr}(3.0$ $\mathrm{M}$ in THF, $3.93 \mathrm{~mL}, 11.8 \mathrm{mmol})$ were reacted in THF $(24 \mathrm{~mL})$. The reaction did not require further purification to give title compound S24 (1.94 g, 99\%) as an orange oil, with spectroscopic data in accordance with the literature. ${ }^{3}{ }^{1} \mathrm{H}$ NMR $\left(400 \mathrm{MHz}, \mathrm{CDCl}_{3}\right) \delta_{\mathrm{H}}: 7.63-$ 7.57 (m, 1H, ArH), 7.52-7.49 (m, 1H, ArH), 7.39-7.31 (m, 1H, ArH), 7.20-7.05 (m, 1H, ArH), 5.33-5.15 (m, 1H, $\left.\mathrm{CHCH}_{3}\right), 2.17(\mathrm{~s}, 1 \mathrm{H}, \mathrm{OH}), 1.49-1.45\left(\mathrm{~m}, 3 \mathrm{H}, \mathrm{CHCH}_{3}\right)$. 


\section{1-(4-Methoxyphenyl)prop-2-yn-1-ol, S25}<smiles>C#CC(O)c1ccc(OC)cc1</smiles>

Following General Procedure A, 4-methoxybenzaldehyde (1.2 mL, $9.8 \mathrm{mmol})$ and ethynylmagnesium chloride $(0.5 \mathrm{M}$ in THF/Toluene, $24 \mathrm{~mL}, 11.8 \mathrm{mmol})$ were reacted in THF $(24 \mathrm{~mL})$. The crude product was purified by silica-gel column chromatography (petrol/EtOAc, 90:10 to 70:30, $\left.\mathrm{R}_{f}: 0.33\right)$ to give title compound $\mathbf{S 2 5}(1.21 \mathrm{~g}, 76 \%)$ as yellow oil, with spectroscopic data in accordance with the literature. ${ }^{4}{ }^{1} \mathrm{H}$ NMR $\left(300 \mathrm{MHz}, \mathrm{CDCl}_{3}\right) \delta_{\mathrm{H}}: 7.58-$ $7.42(\mathrm{~m}, 2 \mathrm{H}, \operatorname{ArC}(2,6) H), 6.99-6.82(\mathrm{~m}, 2 \mathrm{H}, \operatorname{ArC}(3,5) H), 5.43$ (q, $J=6.2 \mathrm{~Hz}, 1 \mathrm{H}, \operatorname{ArCHOH})$, $3.82\left(\mathrm{~s}, 3 \mathrm{H}, \mathrm{OCH}_{3}\right), 2.67(\mathrm{~d}, J=2.3 \mathrm{~Hz}, 1 \mathrm{H}, \mathrm{CCH})$.

\section{2,2-Dimethyl-1-phenylpropan-1-ol S26}<smiles>CC(C)(C)C(O)c1ccccc1</smiles>

Following General Procedure A, benzaldehyde $(1.04 \mathrm{~mL}, 9.8 \mathrm{mmol})$ and $t$ - $\mathrm{BuMgCl}(1.0 \mathrm{M}$ in THF, $11.8 \mathrm{~mL}, 11.8 \mathrm{mmol})$ were reacted in THF $(15 \mathrm{~mL})$. The crude product was purified by silica-gel column chromatography (petrol/EtOAc, 9.5:0.5 to 90:10, $\mathrm{R}_{f} 0.51$ ) to give title compound $\mathbf{S 2 6}(0.361 \mathrm{~g}, 22 \%)$ as a yellow oil, with spectroscopic data in accordance with the literature. ${ }^{5}{ }^{1} \mathrm{H}$ NMR $\left(400 \mathrm{MHz}, \mathrm{CDCl}_{3}\right) \delta_{\mathrm{H}}: 7.34-7.22(\mathrm{~m}, 5 \mathrm{H}, \mathrm{ArH}), 4.38(\mathrm{~s}, 1 \mathrm{H}, \mathrm{PhCH}), 2.09$ (s, 1H, OH), $0.94\left(\mathrm{~s}, 9 \mathrm{H}, \mathrm{CH}\left(\mathrm{CH}_{3}\right)_{3}\right)$.

\section{1-Phenyl-2-(trimethylsilyl)ethan-1-ol, S27}<smiles>C[As](C)CC(O)c1ccccc1</smiles>

$\mathrm{Mg}(0.37 \mathrm{~g}, 15 \mathrm{mmol})$ was suspended in anhydrous $\mathrm{Et}_{2} \mathrm{O}(2 \mathrm{~mL})$, before two drops of a solution of chloromethyltrimethylsilane $(1.5 \mathrm{~g}, 12 \mathrm{mmol})$ in anhydrous THF solution $(1.7 \mathrm{~mL})$ and a crystal of iodine were added. The suspension was heated with a heat gun until complete discoloration was shown. After the $\mathrm{Mg}$ was activated, the rest of the chloromethyltrimethylsilane solution was added dropwise and the reaction stirred for $1 \mathrm{~h}$ at $\mathrm{rt}$. The freshly prepared Grignard was cooled to $0{ }^{\circ} \mathrm{C}$ before benzaldehyde $(1.0 \mathrm{~mL}, 10 \mathrm{mmol})$ was added and reaction was stirred for $1 \mathrm{~h}$ at $\mathrm{rt}$. The reaction was quenched with $\mathrm{NH}_{4} \mathrm{Cl}$ and extracted with EtOAc $(3 \times 20 \mathrm{~mL})$. The combined organic layers were washed with water, brine, dried over $\mathrm{MgSO}_{4}$, filtered, and concentrated under reduced pressure. The crude product was purified by silica-gel column chromatography (Petrol/EtOAc, 95:5 to 80:20 $\mathrm{R}_{f}$. 0.31) to give 
title compound S27 (0.73 g, 38\%) as a colourless oil, with spectroscopic data in accordance with the literature. ${ }^{6}{ }^{1} \mathrm{H}$ NMR $\left(300 \mathrm{MHz}, \mathrm{CDCl}_{3}\right) \delta_{\mathrm{H}}: 7.40-7.27(\mathrm{~m}, 5 \mathrm{H}, \mathrm{ArCH}), 4.90-4.80(\mathrm{~m}$, $1 \mathrm{H}, \mathrm{CHCOH}), 1.33-1.12\left(\mathrm{~m}, 2 \mathrm{H}, \mathrm{CH}_{2} \mathrm{Si}\right),-0.08$ (s, 9H, $\left.\mathrm{Si}\left(\mathrm{CH}_{3}\right)_{3}\right)$.

\section{1-(3-Iodophenyl)ethan-1-ol, S28}<smiles>CC(O)c1cccc(I)c1</smiles>

Following General Procedure B, 1-(3-iodophenyl)ethan-1-one (1.5 mL, $10 \mathrm{mmoL})$ and $\mathrm{NaBH}_{4}(0.76 \mathrm{~g}, 20 \mathrm{mmol})$ were reacted in $\mathrm{MeOH}(50 \mathrm{~mL})$. The reaction did not require further purification to give title compound $\mathbf{S 2 8}(2.36 \mathrm{~g}, 96 \%)$ as a colourless oil, with spectroscopic data in accordance with the literature. ${ }^{7}{ }^{1} \mathrm{H}$ NMR $\left(300 \mathrm{MHz}, \mathrm{CDCl}_{3}\right) \delta_{\mathrm{H}}: 7.83-7.76(\mathrm{~m}, 1 \mathrm{H}$, $\operatorname{ArCH}), 7.61-7.53(\mathrm{~m}, 1 \mathrm{H}, \operatorname{ArCH}), 7.44-7.31$ (m, 1H, $\mathrm{ArCH}), 6.97$ (td, $J=7.5,1.8 \mathrm{~Hz}, 1 \mathrm{H}$, $\operatorname{ArCH}), 5.07$ (q, $\left.J=6.4 \mathrm{~Hz}, 1 \mathrm{H}, \mathrm{CHCH}_{3}\right), 1.46\left(\mathrm{~d}, J=6.4 \mathrm{~Hz}, 3 \mathrm{H}, \mathrm{CHCH}_{3}\right)$.

\section{1-(2-Iodophenyl)ethan-1-ol, S29}<smiles>CC(O)c1ccccc1I</smiles>

Following General Procedure B, 1-(2-iodophenyl)ethan-1-one (1.5 mL, $10 \mathrm{mmol})$ and $\mathrm{NaBH}_{4}$ $(0.76 \mathrm{~g}, 20 \mathrm{mmol})$ were reacted in $\mathrm{MeOH}(50 \mathrm{~mL})$. The reaction did not require further purification to give title compound $\mathbf{S 2 9}(2.35 \mathrm{~g}, 95 \%)$ as a colourless oil, with spectroscopic data in accordance with the literature. ${ }^{8}{ }^{1} \mathrm{H}$ NMR $\left(300 \mathrm{MHz}, \mathrm{CDCl}_{3}\right) \delta_{\mathrm{H}}: 7.77-7.71(\mathrm{~m}, 1 \mathrm{H}$, $\mathrm{ArCH}), 7.60-7.52(\mathrm{~m}, 1 \mathrm{H}, \mathrm{ArCH}), 7.41-7.30(\mathrm{~m}, 1 \mathrm{H}, \mathrm{ArCH}), 7.08(\mathrm{t}, J=7.8 \mathrm{~Hz}, 1 \mathrm{H}, \mathrm{ArCH})$, $4.85\left(\mathrm{q}, J=6.4 \mathrm{~Hz}, 1 \mathrm{H}, \mathrm{CHCH}_{3}\right), 1.48\left(\mathrm{~d}, J=6.5 \mathrm{~Hz}, 3 \mathrm{H}, \mathrm{CHCH}_{3}\right)$.

\section{1-(4-Methoxyphenyl)-2,2-dimethylpropan-1-ol, S30}<smiles>COc1ccc(C(O)C(C)(C)C)cc1</smiles>

Following General Procedure A, 4-methoxybenzaldehyde (1.2 mL, $9.8 \mathrm{mmol})$ and $t$ - $\mathrm{BuMgCl}$ (1.7 $\mathrm{M}$ in THF, $7 \mathrm{~mL}, 11.8 \mathrm{mmol})$ were reacted in THF $(24 \mathrm{~mL})$. The crude product was purified by silica-gel column chromatography (petrol/EtOAc, 95:5 to 85:15, Rf: 0.39 ) to give title compound S30 (1.54 g, 81\%) as yellow oil, with spectroscopic data in accordance with the literature. ${ }^{9}{ }^{1} \mathrm{H}$ NMR $\left(300 \mathrm{MHz}, \mathrm{CDCl}_{3}\right) \delta_{\mathrm{H}}: 7.25-7.21(\mathrm{~m}, 2 \mathrm{H}, \operatorname{ArC}(2,6) H), 6.88-6.83(\mathrm{~m}, 2 \mathrm{H}$, 
$\operatorname{ArC}(3,5) H), 4.36(\mathrm{~s}, 1 \mathrm{H}, \mathrm{ArCHOH}), 3.81\left(\mathrm{~s}, 3 \mathrm{H}, \mathrm{OCH}_{3}\right), 0.91\left(\mathrm{~s}, 9 \mathrm{H}, \mathrm{C}\left(\mathrm{CH}_{3}\right)_{3}\right)$.

2-(4-Methoxyphenyl)propan-2-ol, S31

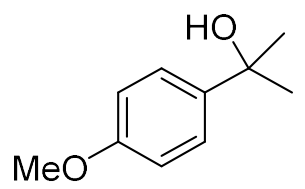

Following General Procedure A, 1-(4-methoxyphenyl)ethan-1-one (1.2 mL, $9.8 \mathrm{mmol})$ and $\mathrm{MeMgCl}\left(3 \mathrm{M}_{\text {in }} \mathrm{Et}_{2} \mathrm{O}, 4 \mathrm{~mL}, 11.8 \mathrm{mmol}\right)$ were reacted in $\mathrm{Et}_{2} \mathrm{O}(24 \mathrm{~mL})$. The reaction did not require further purification to give title compound S31 (1.45 g, 90\%) as a colourless oil, with spectroscopic data in accordance with the literature. ${ }^{10}{ }^{1} \mathrm{H}$ NMR $\left(300 \mathrm{MHz}, \mathrm{CDCl}_{3}\right) \delta_{\mathrm{H}}: 7.47-$ $7.38(\mathrm{~m}, 2 \mathrm{H}, \operatorname{ArC}(2,6) H), 6.93-6.81(\mathrm{~m}, 2 \mathrm{H}, \operatorname{ArC}(3,5) H), 3.81\left(\mathrm{~s}, 3 \mathrm{H}, \mathrm{OCH}_{3}\right), 1.57(\mathrm{~s}, 6 \mathrm{H}$, $\left.\mathrm{C}\left(\mathrm{CH}_{3}\right)_{2}\right)$.

\section{1-Phenylprop-2-en-1-ol S11}<smiles>C=CC(O)c1ccccc1</smiles>

Following General Procedure A, benzaldehyde (1.04 mL, $9.8 \mathrm{mmol})$ and vinylmagnesium bromide $(0.7 \mathrm{M}$ in THF, $17.0 \mathrm{~mL}, 11.8 \mathrm{mmol})$ were reacted in THF $(10 \mathrm{~mL})$. The reaction did not require further purification to give title compound S11 $(1.31 \mathrm{~g}, 99 \%)$ as an orange oil, with spectroscopic data in accordance with the literature. ${ }^{11}{ }^{1} \mathrm{H}$ NMR $\left(400 \mathrm{MHz}, \mathrm{CDCl}_{3}\right) \delta_{\mathrm{H}}: 7.41-$ 7.33 (m, 4H, ArH), 7.33-7.26 (m, 1H, $\mathrm{ArH}), 6.11-6.00$ (m, 1H, $\left.\mathrm{CHCH}_{2}\right)$, 5.39-5.37 (m, 1H, $\mathrm{PhCHOH}), 5.23-5.18\left(\mathrm{~m}, 2 \mathrm{H}, \mathrm{CHCH}_{2}\right), 2.02(\mathrm{~s}, 1 \mathrm{H}, \mathrm{OH})$. 


\section{1,3-Diketones}

All other 1,3-diketones were used as received from commercial sources.

\section{1,3-Bis(4-(trifluoromethyl)phenyl)propane-1,3-dione, S32}

$$
\underset{\mathrm{Ar}=4-\left(\mathrm{F}_{3} \mathrm{C}\right) \mathrm{C}_{6} \mathrm{H}_{4}}{\stackrel{\mathrm{MeOH}(1 \text { equiv. })}{\mathrm{Et}_{3} \mathrm{~N}(2 \text { equiv. })}}
$$

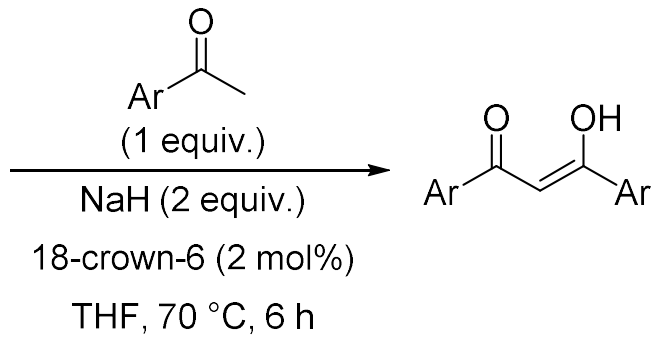

4-(Trifluoromethyl)benzoyl chloride (2.5 g, $12 \mathrm{mmol}$ ) was added slowly to a solution of $\mathrm{MeOH}$ $(0.5 \mathrm{~mL}, 12.0 \mathrm{mmol})$ and $\mathrm{Et}_{3} \mathrm{~N}(3.5 \mathrm{~mL}, 24 \mathrm{mmol})$ were dissolved in anhydrous $\mathrm{CH}_{2} \mathrm{Cl}_{2}(36$ $\mathrm{mL}$ ) under a $\mathrm{N}_{2}$ atmosphere. The reaction was stirred at $\mathrm{rt}$ for $19 \mathrm{~h}$ before being quenched with aq. $1 \mathrm{M} \mathrm{HCl}(10 \mathrm{~mL})$. The layers were separated and the aqueous extracted with $\mathrm{CH}_{2} \mathrm{Cl}_{2}(3 \times 50$ $\mathrm{mL}$ ). The combined organics were washed successively with aq. $\mathrm{NaHCO}_{3}$, aq. $\mathrm{CuSO}_{4}$, and brine before being dried over $\mathrm{MgSO}_{4}$, filtered, and concentrated at reduced pressure to give methyl 4-(trifluoromethyl)benzoate $(1.79 \mathrm{~g}, 73 \%)$ as a yellow oil, with spectroscopic data in accordance with the literature. ${ }^{12}{ }^{1} \mathrm{H}$ NMR $\left(400 \mathrm{MHz}, \mathrm{CDCl}_{3}\right) \delta_{\mathrm{H}}: 8.16(\mathrm{~d}, J=8.0 \mathrm{~Hz}, 2 \mathrm{H}$, $\operatorname{ArC}(3,5) H), 7.71(\mathrm{~d}, J=8.2 \mathrm{~Hz}, 2 \mathrm{H}, \operatorname{ArC}(2,6) H), 3.96\left(\mathrm{~s}, 3 \mathrm{H}, \mathrm{OCH}_{3}\right)$.

Methyl 4-(trifluoromethyl)benzoate $(1.22 \mathrm{~g}, 6.0 \mathrm{mmol})$ was dissolved in anhydrous THF (2 $\mathrm{mL})$ under an $\mathrm{Ar}$ atmosphere before $\mathrm{NaH}(60 \%$ dispersion in mineral oil, $0.24 \mathrm{~g}, 6.0 \mathrm{mmol})$ was added. Absolute ethanol (2 drops) was added followed by a solution of 1-(4(trifluoromethyl)phenyl)ethan-1-one $(0.57 \mathrm{~g}, 3.0 \mathrm{mmol})$ in anhydrous THF $(2 \mathrm{~mL})$ and then a solution of 18-crown-6 $(16 \mathrm{mg}, 0.06 \mathrm{mmol})$ in anhydrous THF $(2 \mathrm{~mL})$. The reaction was stirred at $\mathrm{rt}$ for $30 \mathrm{~min}$ before being heated at reflux for $6 \mathrm{~h}$. The solvent was removed under reduced pressure and the crude purified by silica-gel column chromatography (Petrol/PhMe, 50:50, $\mathrm{R} f$. $0.68)$ to give title compound $\mathbf{S 3 2}(0.45 \mathrm{~g}, 45 \%)$ as an orange solid, with spectroscopic data in accordance with the literature. ${ }^{13} \mathrm{mp} 124-126{ }^{\circ} \mathrm{C}\left\{\right.$ Lit. $\left.^{13} 130^{\circ} \mathrm{C}\right\} ;{ }^{1} \mathrm{H}$ NMR $\left(400 \mathrm{MHz}, \mathrm{CDCl}_{3}\right)$ $\delta_{\mathrm{H}}: 8.10(\mathrm{~d}, J=8.1 \mathrm{~Hz}, 4 \mathrm{H}, \operatorname{ArC}(3,5) H), 7.77(\mathrm{~d}, J=8.2 \mathrm{~Hz}, 4 \mathrm{H}, \operatorname{ArC}(2,6) H), 6.88(\mathrm{~s}, 1 \mathrm{H}, \mathrm{CH})$. 


\section{Silanes}

Allyltrimethylsilane $\mathbf{2 6}$ was used as received from commercial sources.

\section{Cinnamyltrimethylsilane, S33}

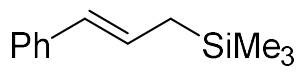

$\mathrm{Mg}(0.23 \mathrm{~g}, 9.20 \mathrm{mmol})$ was suspended in anhydrous THF $(2 \mathrm{~mL})$ before two drops of a solution of cinnamyl chloride $(0.70 \mathrm{~g}, 4.60 \mathrm{mmol})$ in anhydrous THF $(0.6 \mathrm{~mL})$ and a crystal of iodine were added. The suspension was heated with a heat gun until complete discoloration was shown. After the $\mathrm{Mg}$ was activated, the reaction was cooled to $-78^{\circ} \mathrm{C}$ and the rest of the cinnamyl chloride solution was added dropwise followed by addition of TMSCl (2.4 mL, 18.4 mmol) and the reaction was stirred at $-78{ }^{\circ} \mathrm{C}$ for $2 \mathrm{~h}$ followed by $30 \mathrm{~min}$ at $\mathrm{rt}$. The reaction was quenched with cold water and extracted with EtOAc $(3 \times 20 \mathrm{~mL})$. The combined organic layers were washed with brine, dried over $\mathrm{MgSO}_{4}$, filtered, and concentrated under reduced pressure. The reaction did not require further purification to give title compound $\mathbf{S 3 3}(0.80 \mathrm{~g}, 91 \%)$ as a colourless oil, with spectroscopic data in accordance with the literature. ${ }^{14}{ }^{1} \mathrm{H}$ NMR $(300 \mathrm{MHz}$, $\left.\mathrm{CDCl}_{3}\right) \delta_{\mathrm{H}}: 7.35-7.27(\mathrm{~m}, 4 \mathrm{H}, \operatorname{ArCH}), 7.20-7.12(\mathrm{~m}, 1 \mathrm{H}, \operatorname{ArC}(4) H), 6.26-6.22(\mathrm{~m}, 2 \mathrm{H}$, $\mathrm{CHCH}), 1.71-1.63\left(\mathrm{~m}, 2 \mathrm{H}, \mathrm{CHCH}_{2}\right), 0.05\left(9 \mathrm{H}, \mathrm{s}, \mathrm{Si}\left(\mathrm{CH}_{3}\right)_{3}\right)$. 


\section{Dehydrative Alkylation of 1,3-Diketone Derivatives}

General Procedure C: Dehydrative alkylation of 1,3-diketone derivatives

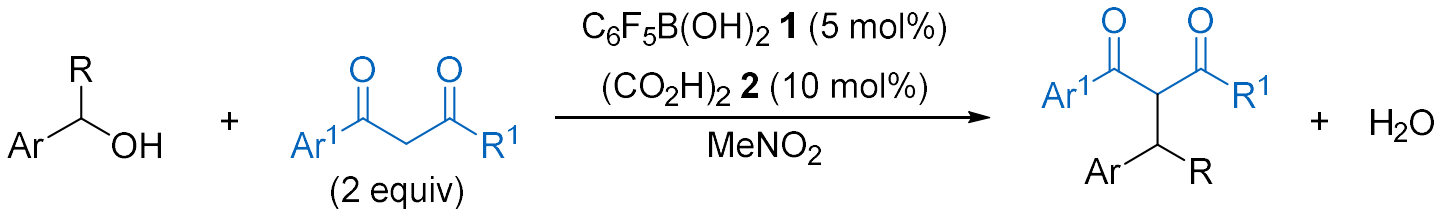

The required nucleophile (2.0 equiv.) was added to a solution of pentafluorophenylboronic acid $1(5 \mathrm{~mol} \%)$ and oxalic acid $2(10 \mathrm{~mol} \%)$ in $\mathrm{MeNO}_{2}(0.05 \mathrm{M})$ and was stirred at $\mathrm{rt}$ for $5 \mathrm{mins}$. The required benzylic alcohol (1.0 equiv.) was added and the reaction stirred at the stated temperature until complete by TLC analysis. The reaction was cooled to rt, diluted with toluene and concentrated under reduced pressure. The crude product was purified by silica-gel column chromatography.

\section{2-Benzhydryl-1,3-diphenylpropane-1,3-dione 3}<smiles>O=C(C(c1ccccc1)c1ccccc1)C(c1ccccc1)c1ccccc1</smiles>

Following General Procedure C, benzhydrol (74 mg, $0.4 \mathrm{mmol}$ ), 1,3-diphenylpropane-1,3dione (179 mg, $0.8 \mathrm{mmol})$, pentafluorophenylboronic acid 1 (4.2 mg, $20 \mu \mathrm{mol})$ and oxalic acid $2(3.6 \mathrm{mg}, 40 \mu \mathrm{mol})$ in MeNO $(8.0 \mathrm{~mL})$ were reacted at $\mathrm{rt}$ for $3 \mathrm{~h}$. The crude was purified by trituration with cold $\mathrm{Et}_{2} \mathrm{O}$ to give title compound $3(119 \mathrm{mg}, 76 \%)$ as a fine white powder, with spectroscopic data in accordance with the literature. ${ }^{15} \mathrm{mp} 226-227{ }^{\circ} \mathrm{C}\left\{\right.$ Lit. $\left.{ }^{15} 227-229{ }^{\circ} \mathrm{C}\right\} ;{ }^{1} \mathrm{H}$ $\operatorname{NMR}\left(500 \mathrm{MHz}, \mathrm{CDCl}_{3}\right) \delta_{\mathrm{H}}:$ 7.91-7.82 (m, 4H, $\left.\mathrm{ArCH}\right), 7.55-7.45$ (m, 2H, $\left.\mathrm{ArCH}\right), 7.40-7.32$ (m, 4H, ArCH), 7.27 (d, $J=7.2 \mathrm{~Hz}, 4 \mathrm{H}, \operatorname{ArCH}), 7.17$ (t, $J=7.7 \mathrm{~Hz}, 4 \mathrm{H}, \operatorname{ArCH}), 7.11-7.04$ (m, 2H, $\mathrm{ArCH}), 6.38(\mathrm{~d}, J=11.6 \mathrm{~Hz}, 1 \mathrm{H}, \mathrm{OCC} H), 5.35$ (d, $J=11.6 \mathrm{~Hz}, 1 \mathrm{H}, \mathrm{PhCC} H)$.

\section{2-Benzhydryl-1,3-bis(4-methoxyphenyl)propane-1,3-dione 4}<smiles>COc1ccc(C(=O)C(C(=O)c2ccc(OC)cc2)C(c2ccccc2)c2ccccc2)cc1</smiles>

In a modified version of General Procedure C, benzhydrol (74 mg, 0.4 mmol), 1,3-bis(4methoxyphenyl)propane-1,3-dione (570 mg, $2.0 \mathrm{mmol}, 5$ equiv.), pentafluorophenylboronic acid $1(4.2 \mathrm{mg}, 20 \mu \mathrm{mol})$ and oxalic acid $2(3.6 \mathrm{mg}, 40 \mu \mathrm{mol})$ in $\mathrm{MeNO}_{2}(8.0 \mathrm{~mL})$ were reacted at $\mathrm{rt}$ for $24 \mathrm{~h}$. The crude product was purified by silica-gel column chromatography 
(petrol/EtOAc, 95:5, $\left.\mathrm{R}_{f}: 0.13\right)$ to give title compound 4 (107 $\left.\mathrm{mg}, 59 \%\right)$ as a yellow solid, with spectroscopic data in accordance with the literature. $.^{15} \mathrm{mp} 182-183{ }^{\circ} \mathrm{C}\left\{\right.$ Lit. $\left.{ }^{15} 182-183.5{ }^{\circ} \mathrm{C}\right\}$; ${ }^{1} \mathrm{H}$ NMR (500 MHz, $\mathrm{CDCl}_{3}$ ) $\delta_{\mathrm{H}}:$ 7.93-7.85 (m, 4H, ArCH), 7.30-7.24 (m, 4H, ArCH), 7.13 (m, 4H, ArCH), 7.10-7.05 (m, 2H, ArCH), 6.87-6.80 (m, 4H, ArCH), 6.24 (d, J=11.7 Hz, 1H, $\operatorname{ArCOCH}), 5.35(\mathrm{~d}, J=11.7 \mathrm{~Hz}, 1 \mathrm{H}, \mathrm{PhCCH}), 3.83\left(\mathrm{~s}, 6 \mathrm{H}, 2 \times \mathrm{OCH}_{3}\right)$.

\section{2-Benzhydryl-1,3-bis(4-(trifluoromethyl)phenyl)propane-1,3-dione 5}<smiles>O=C(c1ccc(C(F)(F)F)cc1)C(C(=O)c1ccc(C(F)(F)F)cc1)C(c1ccccc1)c1ccccc1</smiles>

In a modified version of General Procedure C, benzhydrol (147 mg, $0.8 \mathrm{mmol}, 2$ equiv.), 1,3bis(4-(trifluoromethyl)phenyl)propane-1,3-dione $\mathbf{S 3 2}$ (145 mg, $0.4 \mathrm{mmol}, 1$ equiv.), pentafluorophenylboronic acid $1(4.2 \mathrm{mg}, 20 \mu \mathrm{mol})$ and oxalic acid $2(3.6 \mathrm{mg}, 40 \mu \mathrm{mol})$ in $\mathrm{MeNO}_{2}(8.0 \mathrm{~mL})$ were reacted at $\mathrm{rt}$ for $24 \mathrm{~h}$. The crude product was purified by silica-gel column chromatography (petrol/EtOAc, $95: 5, \mathrm{R}_{f}$. 0.27$)$ to give title compound $\mathbf{5}(167 \mathrm{mg}, 79 \%)$ as a white solid. mp 206-209 ${ }^{\circ} \mathrm{C}$; $v_{\max }$ (solid) $3061(\mathrm{C}-\mathrm{H}), 1699(\mathrm{C}=\mathrm{O}) ;{ }^{1} \mathrm{H}$ NMR $(500 \mathrm{MHz}$, $\left.\mathrm{CDCl}_{3}\right) \delta_{\mathrm{H}}: 7.91(\mathrm{~d}, J=8.1 \mathrm{~Hz}, 4 \mathrm{H}, \mathrm{ArCH}), 7.62(\mathrm{~d}, J=8.1 \mathrm{~Hz}, 4 \mathrm{H}, \mathrm{ArCH}), 7.24-7.22(\mathrm{~m}, 4 \mathrm{H}$, $\operatorname{ArCH}), 7.17$ (t, $J=7.6 \mathrm{~Hz}, 4 \mathrm{H}, \mathrm{ArCH}), 7.11-7.04$ (m, 2H, ArCH), 6.29 (d, $J=11.7 \mathrm{~Hz}, 1 \mathrm{H}$, ArCOCH), $5.30(\mathrm{~d}, J=11.7 \mathrm{~Hz}, 1 \mathrm{H}, \mathrm{PhCC} H) ;{ }^{13} \mathrm{C}\left\{{ }^{1} \mathrm{H}\right\} \mathrm{NMR}\left(126 \mathrm{MHz}, \mathrm{CDCl}_{3}\right) \delta_{\mathrm{C}}: 193.3$ $(\mathrm{ArCCO} \times 2), 141.1(\mathrm{PhCCH} \times 2), 139.5(\mathrm{ArCCO} \times 2), 134.8\left(\mathrm{q},{ }^{2} J_{C-F}=32.9 \mathrm{~Hz}, \operatorname{ArC}(4) \mathrm{CF}_{3}\right)$, $128.9\left(\mathrm{q},{ }^{3} J_{C-F} 4.7, \mathrm{ArCCF}_{3}\right), 128.2(\mathrm{ArCCH} \times 8), 127.2(\mathrm{ArCCH} \times 2), 125.9\left(\mathrm{q},{ }^{3} J_{C-F}=3.5 \mathrm{~Hz}\right.$, $\left.\mathrm{ArCCF}_{3}\right), 123.5\left(\mathrm{q},{ }^{1} J_{\mathrm{C}-\mathrm{F}}=273 \mathrm{~Hz}, \operatorname{ArCF} 3\right), 63.5(\mathrm{ArCOCH}), 52.6(\mathrm{ArCCH}) ;{ }^{19} \mathrm{~F}\left\{{ }^{1} \mathrm{H}\right\} \mathrm{NMR}$ $\left(471 \mathrm{MHz}, \mathrm{CDCl}_{3}\right) \delta_{\mathrm{F}}:-63.26$; HRMS $\left(\mathrm{APCI}^{+}\right) \mathrm{m} / z$ : $\left[\mathrm{M}+\mathrm{NH}_{4}\right]^{+}$calcd $\mathrm{C}_{30} \mathrm{H}_{24} \mathrm{~F}_{6} \mathrm{O}_{2} \mathrm{~N}$ 544.1711, found 544.1710 .

\section{2-(Benzo[d] thiazol-2-yl)-1-(4-methoxyphenyl)-3,3-diphenylpropan-1-one, 6}

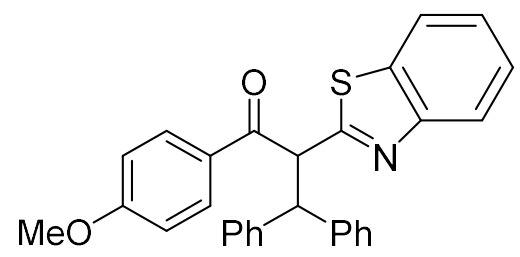

Following General Procedure C, benzhydrol (74 mg, 0.4 mmol), 2-(benzo[d]thiazol-2-yl)-1(4-methoxyphenyl)ethan-1-one (227 mg, $0.8 \mathrm{mmol})$, pentafluorophenylboronic acid 1 (4.2 mg, $20 \mu \mathrm{mol})$ and oxalic acid $2(3.6 \mathrm{mg}, 40 \mu \mathrm{mol})$ in $\mathrm{MeNO}_{2}(8.0 \mathrm{~mL})$ were reacted at $90{ }^{\circ} \mathrm{C}$ for $48 \mathrm{~h}$. The crude product was purified by silica-gel column chromatography (petrol/EtOAc, 90:10 to $\left.70: 30, \mathrm{R}_{f} .0 .23\right)$ to give title compound $\mathbf{6}(151 \mathrm{mg}, 98 \%)$ as a pale brown solid, $\mathrm{mp}$ 
$182-184{ }^{\circ} \mathrm{C} ; v_{\max }\left(\right.$ solid) $3022(\mathrm{C}-\mathrm{H}), 1686(\mathrm{C}=\mathrm{O}) ;{ }^{1} \mathrm{H}$ NMR $\left(500 \mathrm{MHz}, \mathrm{CDCl}_{3}\right) \delta_{\mathrm{H}}$ :

$8.10(\mathrm{~d}, J=8.7 \mathrm{~Hz}, 2 \mathrm{H}, \operatorname{ArC}(2,6) H), 7.92(\mathrm{~d}, J=8.1 \mathrm{~Hz}, 1 \mathrm{H}, \operatorname{ArCH}), 7.74(\mathrm{~d}, J=8.0 \mathrm{~Hz}, 1 \mathrm{H}$, $\mathrm{ArCH}), 7.44-7.33$ (m, 5H, ArCH×5), 7.31-7.24 (m, 1H, $\mathrm{ArCH}), 7.20(\mathrm{t}, J=7.6,2 \mathrm{H}, \mathrm{ArCH})$, 7.15 (t, $J=7.6 \mathrm{~Hz}, 2 \mathrm{H}, \operatorname{ArCH}), 7.12-7.07$ (m, 1H, $\mathrm{ArCH}), 7.06-7.01$ (m, 1H, $\operatorname{ArCH}), 6.89$ (d, $J=8.7 \mathrm{~Hz}, 2 \mathrm{H}, \operatorname{ArC}(3,5) H), 6.30(\mathrm{~d}, J=11.9 \mathrm{~Hz}, 1 \mathrm{H}, \operatorname{ArCCOCH}), 5.23(\mathrm{~d}, J=11.9 \mathrm{~Hz}, 1 \mathrm{H}$, $\mathrm{PhCC} H), 3.81\left(\mathrm{~s}, 3 \mathrm{H}, \mathrm{OCH}_{3}\right) ;{ }^{13} \mathrm{C}\left\{{ }^{1} \mathrm{H}\right\}$ NMR $\left(126 \mathrm{MHz}, \mathrm{CDCl}_{3}\right) \delta_{\mathrm{C}}: 194.6(\mathrm{ArCCO}), 167.9$ $(\mathrm{SArCN}), 164.0(\mathrm{ArC}), 152.2(\operatorname{Het} \mathrm{ArNC}), 142.3(\mathrm{ArCH}), 141.3(\mathrm{ArCH}), 135.7(\operatorname{Het} \mathrm{ArNC})$, 131.4 (ArCH), 129.6 (ArCH), $128.8(\mathrm{ArCH}), 128.6(\mathrm{ArCH}), 127.8(\mathrm{ArCH} \times 2), 126.8(\mathrm{ArCH})$, $126.4(\mathrm{ArCH}), 125.9(\mathrm{ArCH}), 125.1(\mathrm{ArCH}), 123.0(\mathrm{ArCH}), 121.7(\mathrm{ArCH}), 114.0(\mathrm{ArCH})$, $56.8(\mathrm{ArCCOCH}), 55.6\left(\mathrm{OCH}_{3}\right), 54.9(\mathrm{PhCHCH})$; HRMS $\left(\mathrm{APCI}^{+}\right) \mathrm{m} / z:[\mathrm{M}+\mathrm{H}]^{+}$calcd for $\mathrm{C}_{29} \mathrm{H}_{24} \mathrm{NO}_{2} \mathrm{~S} 450.1522$, found 450.1521 .

\section{2-(Benzo[d]oxazol-2-yl)-1,3,3-triphenylpropan-1-one, 7}

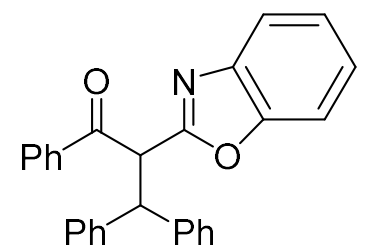

Following General Procedure C, benzhydrol (74 mg, 0.4 mmol), 2-(benzo[d]oxazol-2-yl)-1phenylethan-1-one (191 mg, $0.8 \mathrm{mmol})$, pentafluorophenylboronic acid 1 (4.2 mg, $20 \mu \mathrm{mol})$ and oxalic acid $2(3.6 \mathrm{mg}, 40 \mu \mathrm{mol})$ in $\mathrm{MeNO}_{2}(8.0 \mathrm{~mL})$ were reacted at $90{ }^{\circ} \mathrm{C}$ for $48 \mathrm{~h}$. The crude product was purified by silica-gel column chromatography (petrol/EtOAc, 90:10 to 70:30, $\left.\mathrm{R}_{f}: 0.23\right)$ to give title compound $7(79 \mathrm{mg}, 49 \%)$ as a white solid. $\mathrm{mp} 215-218{ }^{\circ} \mathrm{C} ; v_{\max }$ (film) 3059 (C-H Ar), 3030 (C-H alkane), 1700 (C=O ketone), 1540 (C=C Ar), 1069 (C-N), $937\left(\mathrm{C}=\mathrm{C}\right.$ Ar); ${ }^{1} \mathrm{H}$ NMR $\left(400 \mathrm{MHz}, \mathrm{CDCl}_{3}\right) \delta_{\mathrm{H}}: 8.13-7.98(\mathrm{~m}, 2 \mathrm{H}, \mathrm{ArCH}), 7.62-7.58(\mathrm{~m}, 1 \mathrm{H}$, $\mathrm{ArCH}), 7.56-7.52$ (m, 1H, ArCH), 7.47-7.41 (m, 3H, ArCH×3), 7.38-7.34 (m, 2H, ArCH), 7.34-7.30 (m, 2H, $\mathrm{ArCH}$ ), 7.27-7.20 (m, 4H, ArCH×4), 7.14-7.09 (m, 3H, ArCH×3), 7.07$6.98(\mathrm{~m}, 1 \mathrm{H}, \mathrm{ArCH}), 6.07$ (d, $J=12.0 \mathrm{~Hz}, 1 \mathrm{H}, \mathrm{ArCOCH}), 5.40$ (d, $J=12.0 \mathrm{~Hz}, 1 \mathrm{H}, \mathrm{PhCC} H)$; ${ }^{13} \mathrm{C}\left\{{ }^{1} \mathrm{H}\right\}$ NMR $\left(126 \mathrm{MHz}, \mathrm{CDCl}_{3}\right) \delta_{\mathrm{C}}: 193.2(\mathrm{ArCCO}), 161.7(\mathrm{ArCN}), 150.9(\mathrm{ArC}), 141.6$ $(\mathrm{ArC}), 141.2(\mathrm{ArC}), 140.9(\mathrm{ArC}), 136.3(\mathrm{ArC}), 133.7(\mathrm{ArCH}), 128.8(\mathrm{ArCH}), 128.8(\mathrm{ArCH})$, 128.7 (ArCH), 128.6 (ArCH), $128.0(\mathrm{ArCH}), 127.8(\mathrm{ArCH}), 126.8(\mathrm{ArCH} \times 2), 125.0(\mathrm{ArCH})$, $124.3(\mathrm{ArCH}), 120.0(\mathrm{ArCH}), 110.6(\mathrm{ArCH}), 52.6(\mathrm{ArCOCH}), 51.8(\mathrm{PhCCH}) ; \mathrm{HRMS}\left(\mathrm{NSI}^{+}\right)$ $m / z:[\mathrm{M}+\mathrm{H}]^{+}$calcd for $\mathrm{C}_{28} \mathrm{H}_{22} \mathrm{O}_{2} \mathrm{~N}$ 404.1645, found 404.1645. 


\section{2-Benzhydryl-2-methylcyclopentane-1,3-dione, 8}

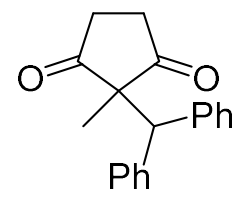

Following General Procedure C, benzhydrol (74 mg, $0.4 \mathrm{mmol}, 50 \mu \mathrm{L}$ ), 2methylcyclopentane-1,3-dione (90 mg, $0.8 \mathrm{mmol})$, pentafluorophenylboronic acid 1 (4.2 $\mathrm{mg}$, $20 \mu \mathrm{mol})$ and oxalic acid $2(3.6 \mathrm{mg}, 40 \mu \mathrm{mol})$ in $\mathrm{MeNO}_{2}(8.0 \mathrm{~mL})$ were reacted at $90{ }^{\circ} \mathrm{C}$ for $16 \mathrm{~h}$. The crude product was purified by silica-gel column chromatography (petrol/EtOAc, 95:5 to $\left.80: 20 \mathrm{R}_{f} .0 .23\right)$ to give title compound $\mathbf{8}(103 \mathrm{mg}, 93 \%)$ as a light yellow solid. mp $132-133$ ${ }^{\circ} \mathrm{C}$; $v_{\max } 3030(\mathrm{C}-\mathrm{H}), 2917(\mathrm{C}-\mathrm{H}), 1716(\mathrm{C}=\mathrm{C}), 1598(\mathrm{C}=\mathrm{C}) ;{ }^{1} \mathrm{H}$ NMR $\left(300 \mathrm{MHz}, \mathrm{CDCl}_{3}\right) \delta_{\mathrm{H}}$ : 7.47-7.41 (m, 4H, ArCH), 7.31-7.27 (m, 2H, ArCH), 7.26-7.18 (m, 4H, ArCH), 4.36 (s, 1H, $\mathrm{PhCCH}$ ), 2.67-2.48 (m, 2H, $\left.\mathrm{CH}_{2} \mathrm{CH}_{2}\right), 2.13-2.05\left(\mathrm{~m}, 2 \mathrm{H}, \mathrm{CH}_{2} \mathrm{CH}_{2}\right), 1.11\left(\mathrm{~s}, 3 \mathrm{H}, \mathrm{CH}_{3}\right)$; ${ }^{13} \mathrm{C}\left\{{ }^{1} \mathrm{H}\right\}$ NMR $\left(126 \mathrm{MHz}, \mathrm{CDCl}_{3}\right) \delta_{\mathrm{C}}: 217.8(\mathrm{CO}), 139.7(\mathrm{ArC}(1)), 129.7(\mathrm{ArCH}), 128.7$ (ArCH), $127.3(\mathrm{ArCH}), 60.5\left(\mathrm{CCH}_{3}\right), 58.5$ (ArCHAr), $36.1\left(\mathrm{CH}_{2} \mathrm{CH}_{2}\right), 20.2\left(\mathrm{CCH}_{3}\right)$; HRMS $\left(\mathrm{ESI}^{-}\right) \mathrm{m} / z:[\mathrm{M}-\mathrm{H}]^{-}$calcd for $\mathrm{C}_{19} \mathrm{H}_{17} \mathrm{O}_{2}{ }^{-} 277.1234$, found 277.1234 .

\section{1,3-Diphenyl-2-(1-phenylethyl)propane-1,3-dione, 9}<smiles>CC(c1ccccc1)C(C(=O)c1ccccc1)c1ccccc1</smiles>

Following General Procedure C, 1-phenylethan-1-ol S18 (52 mg, 0.4 mmol), 1,3diphenylpropane-1,3-dione 2 (180 mg, $0.8 \mathrm{mmol})$, pentafluorophenylboronic acid 1 (4.2 $\mathrm{mg}$, $20 \mu \mathrm{mol})$ and oxalic acid $2(3.6 \mathrm{mg}, 40 \mu \mathrm{mol})$ in $\mathrm{MeNO}_{2}(8.0 \mathrm{~mL})$ were reacted at $90{ }^{\circ} \mathrm{C}$ for $24 \mathrm{~h}$. The crude product was purified by silica-gel column chromatography (petrol/EtOAc, 93:7 $\mathrm{R}_{f: 0.30)}$ to give title compound $9(123 \mathrm{mg}, 88 \%)$ as a white solid, with spectroscopic data in accordance with the literature. ${ }^{16} \mathrm{mp} 120-122{ }^{\circ} \mathrm{C}\left\{\right.$ Lit. $\left.^{16}{ }^{128-129}{ }^{\circ} \mathrm{C}\right\} ;{ }^{1} \mathrm{H}$ NMR $(400 \mathrm{MHz}$, $\left.\mathrm{CDCl}_{3}\right) \delta_{\mathrm{H}}: 8.13-8.02(\mathrm{~m}, 2 \mathrm{H}, \mathrm{ArCHCO}), 7.79-7.74(\mathrm{~m}, 2 \mathrm{H}, \mathrm{ArCHCO}), 7.62-7.56(\mathrm{~m}, 1 \mathrm{H}$, ArCHCO), 7.50-7.42 (m, 1H, ArCHCO), 7.34-7.27 (m, 4H, ArCH and ArCHCO), 7.23-7.17 (m, 2H, ArCH), 7.15-7.07 (m, 1H, ArCH), 5.63 (d, J=10.1 Hz, 1H, PhCOCH), 4.17-4.04 (m, 1H, $\mathrm{PhCCH}), 1.37$ (d, $\left.J=7.0 \mathrm{~Hz}, 3 \mathrm{H}, \mathrm{CHCH}_{3}\right)$.

Gram scale: Following General Procedure C, 1-phenylethan-1-ol S18 (0.49 mL, 4.00 mmol), 1,3-diphenylpropane-1,3-dione (1.79 g, $8.00 \mathrm{mmol})$, pentafluorophenylboronic acid 1 (42 $\mathrm{mg}$, $0.20 \mathrm{mmol})$ and oxalic acid $2(36 \mathrm{mg}, 0.400 \mathrm{mmol})$ in $\mathrm{MeNO}_{2}(80 \mathrm{~mL})$ were reacted at $90{ }^{\circ} \mathrm{C}$ for $19 \mathrm{~h}$. The crude product was purified by silica-gel column chromatography (petrol/EtOAc, 
93:7 $\left.\mathrm{R}_{f}: 0.30\right)$ to give title compound $9(1.225 \mathrm{~g}, 93 \%)$ as a white powder, with spectroscopic data as above. Excess 1,3-diphenylpropane-1,3-dione was also recovered from the column $\left(\mathrm{R}_{f} .0 .53,0.616 \mathrm{~g}, 69 \%\right)$ as a pale orange powder.

\section{2-(1-(4-Methoxyphenyl)ethyl)-1,3-diphenylpropane-1,3-dione, 10}<smiles>COc1ccc(C(C)C(C(=O)c2ccccc2)C(=O)c2ccccc2)cc1</smiles>

Following General Procedure C, 1-(4-methoxyphenyl)ethan-1-ol S19 (61 mg, 0.4 mmol), 1,3-diphenylpropane-1,3-dione (180 mg, $0.8 \mathrm{mmol})$, pentafluorophenylboronic acid 1 (4.2 mg, $20 \mu \mathrm{mol})$ and oxalic acid $2(3.6 \mathrm{mg}, 40 \mu \mathrm{mol})$ in $\mathrm{MeNO}_{2}(8.0 \mathrm{~mL})$ were reacted at $90{ }^{\circ} \mathrm{C}$ for $24 \mathrm{~h}$. The crude product was purified by silica-gel column chromatography (petrol/EtOAc, 93:7 $\mathrm{R}_{f}$ :0.26) to give title compound $\mathbf{1 0}(103 \mathrm{mg}, 70 \%)$ as an off-white crystalline solid, with spectroscopic data in accordance with the literature. ${ }^{16} \mathrm{mp} 106-108{ }^{\circ} \mathrm{C}\left\{\right.$ Lit. $\left.^{16} 109-113{ }^{\circ} \mathrm{C}\right\} ;{ }^{1} \mathrm{H}$ NMR (400 MHz, $\left.\mathrm{CDCl}_{3}\right) \delta_{\mathrm{H}}:$ 8.14-7.95 (m, 2H, $\left.\mathrm{ArCHCO}\right), 7.79-7.69$ (m, 2H, ArCHCO), 7.61-7.50 (m, 1H, ArCHCO), 7.49-7.37 (m, 3H, ArCHCO), 7.32-7.23 (m, 2H, ArCHCO), 7.21-7.12 (m, 2H, $\operatorname{ArC}(2,6) H), 6.80-6.65(\mathrm{~m}, 2 \mathrm{H}, \operatorname{ArC}(3,5) H), 5.55(\mathrm{~d}, J=10.1 \mathrm{~Hz}, 1 \mathrm{H}$, $\operatorname{ArCOCH}), 4.04(\mathrm{dq}, J=10.1,7.0 \mathrm{~Hz}, 1 \mathrm{H}, \mathrm{ArCC} H), 3.70\left(\mathrm{~s}, 3 \mathrm{H}, \mathrm{OCH}_{3}\right), 1.32$ (d, J=7.0 Hz, $\left.3 \mathrm{H}, \mathrm{CHCH}_{3}\right)$.

\section{2-(1-(2-Methoxyphenyl)ethyl)-1,3-diphenylpropane-1,3-dione, 11}<smiles>COc1ccccc1C(C)C(C(=O)c1ccccc1)C(=O)c1ccccc1</smiles>

Following General Procedure C, 1-(2-methoxyphenyl)ethan-1-ol S20 (61 mg, 0.4 mmol), 1,3-diphenylpropane-1,3-dione (180 mg, $0.8 \mathrm{mmol}$ ), pentafluorophenylboronic acid 1 (4.2 $\mathrm{mg}$, $20 \mu \mathrm{mol})$ and oxalic acid $2(3.6 \mathrm{mg}, 40 \mu \mathrm{mol})$ in $\mathrm{MeNO}_{2}(8.0 \mathrm{~mL})$ were reacted at $40{ }^{\circ} \mathrm{C}$ for $48 \mathrm{~h}$. The crude product was purified by silica-gel column chromatography (petrol/EtOAc, 100:0 to 85:15 $\left.\mathrm{R}_{f} .0 .35\right)$ to give title compound $11(136 \mathrm{mg}, 95 \%)$ as colorless oil. $v_{\max }$ (film) 3062 (C-H Ar), 2962 (C-H alkane), 1693 (C=O Ketone), 1662 (C=C Ar), 1250 (C-O ether), 810 (C-H Ar); ${ }^{1} \mathrm{H}$ NMR (400 MHz, $\left.\mathrm{CDCl}_{3}\right) \delta_{\mathrm{H}}:$ 8.07-7.95 (m, 2H, $\left.\mathrm{ArCH}\right), 7.92-7.81(\mathrm{~m}, 2 \mathrm{H}$, ArCH), 7.61-7.52 (m, 3H, ArCH), 7.52-7.41 (m, 3H, PhCH), 7.38-7.31 (m, 2H, ArCH), 7.25- 
$7.20(\mathrm{~m}, 1 \mathrm{H}, \mathrm{ArCH}), 7.15-7.07$ (m, 1H, ArCH), 6.86-6.78 (m, 1H, ArCH), 6.78-6.72 (m, 1H, $\operatorname{ArCH}), 5.99(\mathrm{~d}, J=8.8 \mathrm{~Hz}, 1 \mathrm{H}, \operatorname{ArCOCH}), 4.31-4.14(\mathrm{~m}, 1 \mathrm{H}, \operatorname{ArCCH}), 3.86\left(\mathrm{~s}, 3 \mathrm{H}, \mathrm{OCH}_{3}\right)$, $1.37\left(\mathrm{~d}, J=7.1 \mathrm{~Hz}, 3 \mathrm{H}, \mathrm{CHCH}_{3}\right) ;{ }^{13} \mathrm{C}\left\{{ }^{1} \mathrm{H}\right\} \mathrm{NMR}\left(126 \mathrm{MHz}, \mathrm{CDCl}_{3}\right) \delta_{\mathrm{C}}: 196.0(\mathrm{CO}), 195.5$ $(C \mathrm{O}), 157.2\left(C(2) \mathrm{OCH}_{3}\right), 137.6(\operatorname{ArC}(1)), 137.0(\mathrm{ArC}(1)), 133.3(\operatorname{ArC}(4) \mathrm{H}), 133.0(\operatorname{ArC}(4) \mathrm{H})$, $131.7(\mathrm{ArC}(1)), 128.8(\mathrm{ArC}(2,6) \mathrm{H} \times 2), 128.6(\mathrm{ArC}(3,5) \mathrm{H} \times 2), 127.8(\mathrm{ArC}(4,6) H), 120.8$ $(\mathrm{ArC}(3) H), 110.8(\mathrm{ArC}(5) H), 61.1(\mathrm{OCCH}), 55.2\left(\mathrm{OCH}_{3}\right), 37.03\left(\mathrm{CHCH}_{3}\right), 17.9\left(\mathrm{CHCH}_{3}\right)$; HRMS (APCI ${ }^{+}$) m/z: $[\mathrm{M}+\mathrm{H}]^{+}$calcd for $\mathrm{C}_{24} \mathrm{H}_{23} \mathrm{O}_{3} 359.1642$, found 359.1643 .

\section{2-(1-(4-Fluorophenyl)ethyl)-1,3-diphenylpropane-1,3-dione, 12}<smiles>CC(c1ccc(F)cc1)C(C(=O)c1ccccc1)C(=O)c1ccccc1</smiles>

Following General Procedure C, 1-(4-fluorophenyl)ethan-1-ol S21 (56 mg, 0.4 mmol), 1,3diphenylpropane-1,3-dione (180 mg, $0.8 \mathrm{mmol}$ ), pentafluorophenylboronic acid 1 (4.2 mg, 20 $\mu \mathrm{mol})$ and oxalic acid $2(3.6 \mathrm{mg}, 40 \mu \mathrm{mol})$ in $\mathrm{MeNO}_{2}(8.0 \mathrm{~mL})$ were reacted at $90{ }^{\circ} \mathrm{C}$ for $24 \mathrm{~h}$. The crude product was purified by silica-gel column chromatography (petrol/EtOAc, 100:0 to 85:15 $\left.\mathrm{R}_{f}: 0.21\right)$ to give title compound $12(131 \mathrm{mg}, 90 \%)$ as a white solid, with spectroscopic data in accordance with the literature. ${ }^{17} \mathrm{mp} 109-110{ }^{\circ} \mathrm{C}\left\{\right.$ Lit. $\left.^{17} 110-112{ }^{\circ} \mathrm{C}\right\} ;{ }^{1} \mathrm{H}$ NMR $(400$ $\left.\mathrm{MHz}, \mathrm{CDCl}_{3}\right) \delta_{\mathrm{H}}:$ 8.06-8.00 (m, 2H, $\left.\operatorname{ArCHCO}\right), 7.77-7.71$ (m, 2H, ArCHCO), 7.61-7.53 (m, 1H, ArCHCO), 7.50-7.40 (m, 3H, ArCHCO), 7.35-7.27 (m, 2H, ArCHCO), 7.25-7.18 (m, 2H, $\operatorname{ArC}(2,6) H), 6.90-6.82(\mathrm{~m}, 2 \mathrm{H}, \operatorname{ArC}(3,5) H), 5.54(\mathrm{~d}, J=10.2 \mathrm{~Hz}, 1 \mathrm{H}, \operatorname{ArCCOCH}), 4.16-4.07$ $(\mathrm{m}, 1 \mathrm{H}, \mathrm{ArCC} H), 1.35\left(\mathrm{~d}, J=7.0 \mathrm{~Hz}, 3 \mathrm{H}, \mathrm{CHCH}_{3}\right)$.

\section{2-(1-(4-Bromophenyl)ethyl)-1,3-diphenylpropane-1,3-dione, 13}<smiles>CC(c1ccc(Br)cc1)C(C(=O)c1ccccc1)C(=O)c1ccccc1</smiles>

Following General Procedure C, 1-(4-bromophenyl)ethan-1-ol S22 (81 mg, 0.4 mmol), 1,3diphenylpropane-1,3-dione (180 mg, $0.8 \mathrm{mmol})$, pentafluorophenylboronic acid 1 (4.2 mg, 20 $\mu \mathrm{mol})$ and oxalic acid $2(3.6 \mathrm{mg}, 40 \mu \mathrm{mol})$ in $\mathrm{MeNO}_{2}(8.0 \mathrm{~mL})$ were reacted at $90{ }^{\circ} \mathrm{C}$ for $24 \mathrm{~h}$. The crude product was purified by silica-gel column chromatography (petrol/EtOAc, 100:0 to $\left.85: 15 \mathrm{R}_{f}: 0.21\right)$ to give title compound $\mathbf{1 3}(80 \mathrm{mg}, 71 \%)$ as a white solid, with spectroscopic 
data in accordance with the literature. ${ }^{18} \mathrm{mp} 83-85{ }^{\circ} \mathrm{C}\left\{\right.$ Lit. $\left.{ }^{18} 82-84{ }^{\circ} \mathrm{C}\right\} ;{ }^{1} \mathrm{H}$ NMR $(400 \mathrm{MHz}$, $\left.\mathrm{CDCl}_{3}\right) \delta_{\mathrm{H}}: 8.11-8.01(\mathrm{~m}, 2 \mathrm{H}, \operatorname{ArCH}), 7.84-7.74(\mathrm{~m}, 2 \mathrm{H}, \operatorname{ArC}(3,5) H), 7.67-7.56(\mathrm{~m}, 1 \mathrm{H}$, $\operatorname{ArCH}), 7.56-7.43(\mathrm{~m}, 2 \mathrm{H}, \operatorname{ArCH}), 7.40-7.27$ (m, 4H, $\operatorname{ArCH}), 7.21-7.11(\mathrm{~m}, 2 \mathrm{H}, \operatorname{ArC}(2,6) H)$, $5.55(\mathrm{~d}, J=10.2 \mathrm{~Hz}, 1 \mathrm{H}, \operatorname{ArCOCH}), 4.15-4.00(\mathrm{~m}, 1 \mathrm{H}, \operatorname{ArCCH}), 1.38(\mathrm{~d}, J=7.0 \mathrm{~Hz}, 3 \mathrm{H}$, $\left.\mathrm{CHCH}_{3}\right)$.

\section{2-(1-(3-Bromophenyl)ethyl)-1,3-diphenylpropane-1,3-dione, 14}<smiles>CC(c1cccc(Br)c1)C(C(=O)c1ccccc1)C(=O)c1ccccc1</smiles>

Following General Procedure C, 1-(3-bromophenyl)ethan-1-ol S23 (81 mg, 0.4 mmol), 1,3diphenylpropane-1,3-dione (180 mg, $0.8 \mathrm{mmol}$ ), pentafluorophenylboronic acid 1 (4.2 mg, 20 $\mu \mathrm{mol})$ and oxalic acid $2(3.6 \mathrm{mg}, 40 \mu \mathrm{mol})$ in $\mathrm{MeNO}_{2}(8.0 \mathrm{~mL})$ were reacted at $90{ }^{\circ} \mathrm{C}$ for $24 \mathrm{~h}$. The crude product was purified by silica-gel column chromatography (petrol/EtOAc, 100:0 to 85:15 $\left.\mathrm{R}_{f}: 0.21\right)$ to give title compound 14 (43 mg, 38\%) as a white powder; mp $111-113{ }^{\circ} \mathrm{C}$; $v_{\max }$ (film) 3061 (C-H Ar), 2962 (C-H), 1705 (C=O), 1595 (C=C Ar), 783 (C-H Ar), 758 (C$\mathrm{Br}) ;{ }^{1} \mathrm{H}$ NMR $\left(300 \mathrm{MHz}, \mathrm{CDCl}_{3}\right) \delta_{\mathrm{H}}: 8.12-8.01$ (m, 2H, $\left.\mathrm{ArCH}\right), 7.86-7.74(\mathrm{~m}, 2 \mathrm{H}, \mathrm{ArCH})$, 7.63-7.52 (m, 2H, ArCH), 7.56-7.40 (m, 4H, ArCH), 7.24-7.16 (m, 2H, ArCH), 7.08-7.01 (m, 2H, $\operatorname{ArCH}), 5.59$ (d, $J=10.1 \mathrm{~Hz}, 1 \mathrm{H}, \operatorname{ArCOCH}), 4.21-4.08$ (m, 1H, $\operatorname{ArCCH}), 1.34$ (d, $J=6.9$ $\left.\mathrm{Hz}, 3 \mathrm{H}, \mathrm{CHCH}_{3}\right) ;{ }^{13} \mathrm{C}\left\{{ }^{1} \mathrm{H}\right\} \mathrm{NMR}\left(126 \mathrm{MHz} \mathrm{CDCl}_{3}\right) \delta_{\mathrm{C}}: 194.7$ (CO), $194.4(\mathrm{CO}), 146.3$ (ArC(1)), $136.7(\operatorname{ArC}(1)), 136.7(\operatorname{ArC}(1)), 133.8 \quad(\operatorname{ArC}(4) \mathrm{H}), 133.4 \quad(\operatorname{ArC}(4) \mathrm{H}), 130.8$ $(\operatorname{ArC}(2) H), 130.1(\operatorname{ArC}(5) \mathrm{H}), 129.9(\operatorname{ArC}(4) \mathrm{H}), 129.0(\operatorname{ArC}(2,6) \mathrm{H}), 128.9(\operatorname{ArC}(2,6) \mathrm{H}), 128.7$ $(\mathrm{ArC}(3,5) H), 128.6(\mathrm{ArC}(3,5) \mathrm{H}), 126.8(\mathrm{ArC}(6) \mathrm{H}), 122.5(\mathrm{ArC}(3) \mathrm{Br}), 64.4(\mathrm{ArCOCH}), 40.9$ $\left(\mathrm{CHCH}_{3}\right), 20.2\left(\mathrm{CHCH}_{3}\right)$; HRMS $\left(\mathrm{APCI}^{+}\right) \mathrm{m} / z$ : $[\mathrm{M}+\mathrm{H}]^{+}$calcd for $\mathrm{C}_{23} \mathrm{H}_{20} \mathrm{BrO}_{2} 407.0641$, found 407.0639 . 


\section{2-(1-(2-Bromophenyl)ethyl)-1,3-diphenylpropane-1,3-dione, 15}<smiles>CC(c1ccccc1Br)C(C(=O)c1ccccc1)C(=O)c1ccccc1</smiles>

Following General Procedure C, 1-(2-bromophenyl)ethan-1-ol S24 (89 mg, 0.4 mmol), 1,3diphenylpropane-1,3-dione (180 mg, $0.8 \mathrm{mmol}$ ), pentafluorophenylboronic acid 1 (4.2 mg, 20 $\mu \mathrm{mol})$ and oxalic acid $2(3.6 \mathrm{mg}, 40 \mu \mathrm{mol})$ in $\mathrm{MeNO}_{2}(8.0 \mathrm{~mL})$ were reacted at $90^{\circ} \mathrm{C}$ for $48 \mathrm{~h}$. The crude product was purified by silica-gel column chromatography (petrol/EtOAc, 100:0 to 85:15 $\left.\mathrm{R}_{f}: 0.35\right)$ to give title compound $15(83 \mathrm{mg}, 51 \%)$ as a white solid, with spectroscopic data in accordance with the literature. ${ }^{19} \mathrm{mp} 121-123{ }^{\circ} \mathrm{C}\left\{\right.$ Lit. $\left.{ }^{19} 122-124{ }^{\circ} \mathrm{C}\right\} ;{ }^{1} \mathrm{H}$ NMR (300 $\left.\mathrm{MHz}, \mathrm{CDCl}_{3}\right) \delta_{\mathrm{H}}: 8.01-7.93(\mathrm{~m}, 2 \mathrm{H}, \mathrm{ArCH}), 7.93-7.86(\mathrm{~m}, 2 \mathrm{H}, \mathrm{ArCH}), 7.61-7.48(\mathrm{~m}, 3 \mathrm{H}$, $\mathrm{ArCH}), 7.48-7.40$ (m, 2H, ArCH), 7.40-7.33 (m, 2H, $\mathrm{ArCH}), 7.23-7.18$ (m, 1H, ArCH), 7. 16$7.10(\mathrm{~m}, 1 \mathrm{H}, \operatorname{ArCH}), 7.04-6.97$ (m, 1H, $\operatorname{ArCH}), 5.59$ (d, $J=10.1 \mathrm{~Hz}, 1 \mathrm{H}, \operatorname{ArCOCH}), 4.57-$ $4.43(\mathrm{~m}, 1 \mathrm{H}, \mathrm{ArCCH}), 1.35$ (d, $\left.J=7.1 \mathrm{~Hz}, 3 \mathrm{H}, \mathrm{CHCH}_{3}\right)$.

\section{2-(1-(4-Methoxyphenyl)prop-2-yn-1-yl)-1,3-diphenylpropane-1,3-dione, 16}<smiles>C#CC(c1ccc(OC)cc1)C(C(=O)c1ccccc1)C(=O)c1ccccc1</smiles>

Following General Procedure C, 1-(4-methoxyphenyl)prop-2-yn-1-ol S25 (32 mg, 0.2 mmol), 1,3-diphenylpropane-1,3-dione (90 mg, $0.4 \mathrm{mmol}$ ), pentafluorophenylboronic acid 1 $(2.2 \mathrm{mg}, 10 \mu \mathrm{mol})$ and oxalic acid $2(1.8 \mathrm{mg}, 20 \mu \mathrm{mol})$ in $\mathrm{MeNO}_{2}(4.0 \mathrm{~mL})$ were reacted at $\mathrm{rt}$ for $16 \mathrm{~h}$. The crude product was purified by silica-gel column chromatography (petrol/EtOAc, 100:0 to 80:20 $\left.\mathrm{R}_{f}: 0.22\right)$ to give title compound 16 (72 mg, 98\%) as a white solid. $\mathrm{mp} \mathrm{106-112}$ ${ }^{\circ} \mathrm{C} ; v_{\max } 3030(\mathrm{C}-\mathrm{H}), 2916(\mathrm{C}-\mathrm{H}), 1716(\mathrm{C}=\mathrm{O}), 1597(\mathrm{C}=\mathrm{C}) ;{ }^{1} \mathrm{H} \mathrm{NMR}\left(300 \mathrm{MHz}, \mathrm{CDCl}_{3}\right) \delta_{\mathrm{H}}$ : 8.06-7.98 (m, 2H, ArCHCO), 7.74-7.69 (m, 2H, ArCHCO), 7.61-7.53 (m, 3H, ArCHCO), 7.49-7.41 (m, 3H, ArCHCO), 7.39-7.27 (m, 2H, $\operatorname{ArC}(2,6) H)$, 6.79-6.71 (m, 2H, $\operatorname{ArC}(3,5) H)$, $5.79(\mathrm{~d}, J=10.2 \mathrm{~Hz}, 1 \mathrm{H}, \operatorname{ArCCOCH}), 4.92(\mathrm{dd}, J=10.2,2.5 \mathrm{~Hz}, 1 \mathrm{H}, \operatorname{ArCC} H C C H), 3.71$ (s, $\left.3 \mathrm{H}, \mathrm{OCH}_{3}\right), 2.18(\mathrm{~d}, J=2.5 \mathrm{~Hz}, 1 \mathrm{H}, \mathrm{CHCCH}) ;{ }^{13} \mathrm{C}\left\{{ }^{1} \mathrm{H}\right\} \mathrm{NMR}\left(126 \mathrm{MHz}, \mathrm{CDCl}_{3}\right) \delta_{\mathrm{C}}: 193.3$ (CO), 192.9 (CO), 159 (ArC(4)OMe), 133.7 (ArC(4)H), 133.5 (ArC(4)H), $130.4(\mathrm{ArC}(1))$, $129.7(\mathrm{ArC}(2,6) \mathrm{H}), 128.8(\mathrm{ArCH}), 128.8(\mathrm{ArCH}), 128.8(\mathrm{ArCH}), 128.7(\mathrm{ArCH}), 114.2$ $(\operatorname{ArC}(3,5) \mathrm{H}), 84.3(\mathrm{CCH}), 72.7(\mathrm{CCH}), 63.8(\mathrm{COCHCO}), 55.4\left(\mathrm{OCH}_{3}\right), 37.5(\mathrm{CHC})$; HRMS 
$\left(\mathrm{ESI}^{-}\right) \mathrm{m} / z$ : $[\mathrm{M}-\mathrm{H}]^{-}$calcd for $\mathrm{C}_{24} \mathrm{H}_{22} \mathrm{O}_{4} 374.1518$, found 374.1500 .

\section{3-(Benzhydryloxy)cyclohex-2-en-1-one, S17}<smiles>O=C1C=C(OC(c2ccccc2)c2ccccc2)CCC1</smiles>

Following General Procedure C, cyclohexane-1,3-dione (43 mg, $0.4 \mathrm{mmol}$ ), benzhydrol (150 $\mathrm{mg}, 0.8 \mathrm{mmol})$, pentafluorophenylboronic acid 1 (4.2 $\mathrm{mg}, 20 \mu \mathrm{mol})$ and oxalic acid $2(3.6 \mathrm{mg}$, $40 \mu \mathrm{mol})$ in $\mathrm{MeNO}_{2}(8.0 \mathrm{~mL})$ were reacted at $40{ }^{\circ} \mathrm{C}$ for $16 \mathrm{~h}$. The crude product was purified by silica-gel column chromatography (petrol/EtOAc, 90:10 to 40:60 $\mathrm{R}_{f}$. 0.12 ) to give title compound S17 (81 mg, 73\%) as a yellow oil. $v_{\max } 3029(\mathrm{C}-\mathrm{H}), 2934(\mathrm{C}-\mathrm{H}), 1646(\mathrm{C}=\mathrm{C}), 1610$ $(\mathrm{C}=\mathrm{C}), 1582(\mathrm{C}=\mathrm{C}) ;{ }^{1} \mathrm{H}$ NMR $\left(300 \mathrm{MHz}, \mathrm{CDCl}_{3}\right) \delta_{\mathrm{H}}: 7.39-7.28(\mathrm{~m}, 10 \mathrm{H}, \mathrm{ArCH}), 6.11(\mathrm{~s}, 1 \mathrm{H}$, $\mathrm{OCH}), 5.37$ (s, 1H, $\mathrm{CH}$ ), 2.55 (t, $J=6.2 \mathrm{~Hz}, 2 \mathrm{H}, \mathrm{COCH}_{2} \mathrm{CH}_{2} \mathrm{CH}_{2}$ ), 2.31 (dd, $J=7.3,5.9 \mathrm{~Hz}$, $\left.2 \mathrm{H}, \mathrm{CH}_{2} \mathrm{CH}_{2} \mathrm{CH}_{2} \mathrm{COCH}\right), 2.09-1.91\left(\mathrm{~m}, 2 \mathrm{H}, \mathrm{CH}_{2} \mathrm{CH}_{2} \mathrm{CH}_{2}\right) ;{ }^{13} \mathrm{C}\left\{{ }^{1} \mathrm{H}\right\} \mathrm{NMR}\left(126 \mathrm{MHz}, \mathrm{CDCl}_{3}\right)$ $\delta_{\mathrm{C}}: 199.9(\mathrm{CO}), 176.6(\mathrm{COCHCO}), 144.0(\mathrm{ArC}(1)), 139.8(\mathrm{ArC}(1)), 128.9(\mathrm{ArCH}), 128.7$ $(\mathrm{ArCH}), 128.6(\mathrm{ArCH}), 128.4(\mathrm{ArCH}), 127.7(\mathrm{ArCH}), 127.6(\mathrm{ArCH}), 127.4(\mathrm{ArCH}), 126.8$ $(\mathrm{ArCH}), \quad 105.5$ (COCHCO), 76.4 (ArCHAr), $36.8\left(\mathrm{COCH}_{2}\right), 29.4 \quad\left(\mathrm{COCH}_{2}\right), 20.7$ $\left(\mathrm{CH}_{2} \mathrm{CH}_{2} \mathrm{CH}_{2}\right)$; HRMS (ESI $\left.{ }^{-}\right) \mathrm{m} / z$ : [M-H] $]^{-}$calcd for $\mathrm{C}_{19} \mathrm{H}_{18} \mathrm{O}_{2} 278.1307$, found 278.1302. 


\section{Dehydrative Alkylation of 1,3-Ketoesters}

General Procedure D: Dehydrative alkylation of 1,3-ketoesters

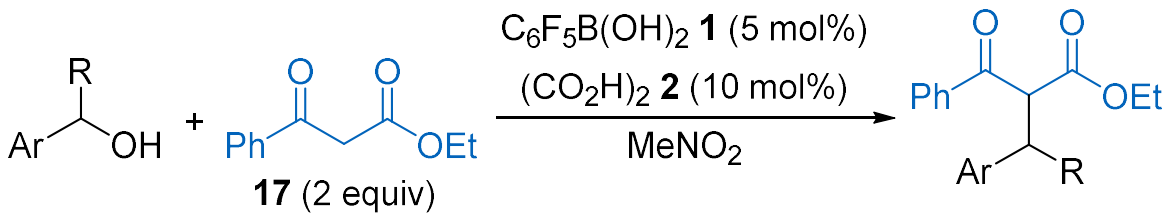

The ethyl benzoylacetate 17 (2.0 equiv.) was added to a solution of pentafluorophenylboronic acid $1(5 \mathrm{~mol} \%)$ and oxalic acid $2(10 \mathrm{~mol} \%)$ in $\mathrm{MeNO}_{2}(0.05 \mathrm{M})$ and was stirred at $\mathrm{rt}$ for 5 mins. The required benzylic alcohol (1.0 equiv.) was added and the reaction stirred at the stated temperature until complete by TLC analysis. The reaction was cooled to rt, diluted with toluene and concentrated under reduced pressure. The crude product was purified by silica-gel column chromatography.

Ethyl 2-benzhydryl-3-oxo-3-phenylpropanoate, 18<smiles>CCOC(=O)C(C(=O)c1ccccc1)c1ccccc1</smiles>

In a modified version of General Procedure D, benzhydrol (147 mg, 0.8 mmol, 2 equiv.), ethyl benzoylacetate 17 ( $80 \mathrm{mg}, 0.4 \mathrm{mmol}, 1$ equiv.), pentafluorophenylboronic acid 1 (4.2 mg, $20 \mu \mathrm{mol})$ and oxalic acid $2(3.6 \mathrm{mg}, 40 \mu \mathrm{mol})$ in $\mathrm{MeNO}_{2}(8.0 \mathrm{~mL})$ were reacted at $90{ }^{\circ} \mathrm{C}$ for $24 \mathrm{~h}$. The crude product was purified by silica-gel column chromatography (petrol/EtOAc, 99:1 to $\left.90: 10, \mathrm{R}_{f}: 0.30\right)$ to give title compound 18 (144 $\left.\mathrm{mg}, 97 \%\right)$ as a white solid, with spectroscopic data in accordance with the literature. ${ }^{15} \mathrm{mp} 133-135{ }^{\circ} \mathrm{C}\left\{\right.$ Lit. $\left.{ }^{15} 138-140{ }^{\circ} \mathrm{C}\right\} ;{ }^{1} \mathrm{H}$ NMR $(400$ $\left.\mathrm{MHz}, \mathrm{CDCl}_{3}\right) \delta_{\mathrm{H}}:$ 8.05-8.01 (m, 2H, $\operatorname{ArCHCO}$ ), 7.59-7.53 (m, 1H, ArCHCO), 7.47-7.37 (m, 4H, ArCH and ArCHCO), 7.32-7.23 (m, 4H, ArCH), 7.22-7.13 (m, 3H, ArCH), 7.09-7.04 (m, $1 \mathrm{H}, \mathrm{ArCH}), 5.43(\mathrm{~d}, J=11.8 \mathrm{~Hz}, 1 \mathrm{H}, \operatorname{ArCCOCH}), 5.10$ (d, $J=11.8 \mathrm{~Hz}, 1 \mathrm{H}, \operatorname{ArCCH}), 3.95-$ $3.90\left(\mathrm{~m}, 2 \mathrm{H}, \mathrm{CH}_{2} \mathrm{CH}_{3}\right), 0.94$ (t, $\left.J=7.1 \mathrm{~Hz}, 3 \mathrm{H}, \mathrm{CH}_{2} \mathrm{CH}_{3}\right)$.

Ethyl 2-benzoyl-3-phenylbutanoate, 19<smiles>CCOC(=O)C(C(=O)c1ccccc1)C(C)c1ccccc1</smiles>

In a modified version of General Procedure D, 1-phenylethan-1-ol S18 (100 mg, 0.8 mmol, 2 equiv.), ethyl benzoylacetate 17 (77 mg, $0.4 \mathrm{mmol}, 1$ equiv.), pentafluorophenylboronic acid 
$1(4.2 \mathrm{mg}, 20 \mu \mathrm{mol})$ and oxalic acid $2(3.6 \mathrm{mg}, 40 \mu \mathrm{mol})$ in $\mathrm{MeNO}_{2}(8.0 \mathrm{~mL})$ were reacted at $90{ }^{\circ} \mathrm{C}$ for $16 \mathrm{~h}$. The crude product was purified by silica-gel column chromatography (petrol/EtOAc, 98:2 to $\left.90: 10 \mathrm{R}_{f} .0 .19\right)$ to give 19 as a mixture of diastereoisomers $(57: 43 \mathrm{dr}$, $211 \mathrm{mg}, 98 \%$ ) as a colourless oil, with spectroscopic data in accordance with the literature. ${ }^{16}$

Data for major diastereoisomer: ${ }^{1} \mathrm{H}$ NMR $\left(300 \mathrm{MHz}, \mathrm{CDCl}_{3}\right)$ (selected) $\delta_{\mathrm{H}}: 8.14-8.07(\mathrm{~m}, 2 \mathrm{H}$, ArCH), 7.64-7.57 (m, 1H, ArCH), 7.55-7.44 (m, 2H, $\mathrm{ArCH}), 7.41-7.28$ (m, 3H, $\mathrm{ArCH}), 7.24-$ 7.06 (m, 2H, ArCH), 4.64 (d, $J=10.6$ Hz, 1H, $\mathrm{ArCCOCH}$ ), 4.26-4.14 (m, 1H, $\mathrm{CHCH}_{3}$ ), 3.89$3.75\left(\mathrm{~m}, 2 \mathrm{H}, \mathrm{CH}_{2} \mathrm{CH}_{3}\right), 1.32-1.25\left(\mathrm{~m}, 3 \mathrm{H}, \mathrm{CHCH}_{3}\right), 0.88\left(\mathrm{t}, J=7.1 \mathrm{~Hz}, 3 \mathrm{H}, \mathrm{OCH}_{2} \mathrm{CH}_{3}\right)$.

Data for minor diastereoisomer: ${ }^{1} \mathrm{H}$ NMR $\left(300 \mathrm{MHz}, \mathrm{CDCl}_{3}\right)$ (selected) $\delta_{\mathrm{H}}: 8.01-7.92(\mathrm{~m}, 2 \mathrm{H}$, ArCH), 7.89-7.80 (m, 2H, ArCH), 7.64-7.57 (m, 1H, ArCH), 7.41-7.28 (m, 3H, ArCH), 7.247.06 (m, 2H, $\mathrm{ArCH}), 4.69$ (d, $J=10.6 \mathrm{~Hz}, 1 \mathrm{H}, \mathrm{ArCCOCH}), 4.26-4.14$ (m, 1H, $\left.\mathrm{CHCH}_{3}\right), 3.89-$ $3.75\left(\mathrm{~m}, 2 \mathrm{H}, \mathrm{CH}_{2} \mathrm{CH}_{3}\right), 1.24-1.19\left(\mathrm{~m}, 3 \mathrm{H}, \mathrm{OCH}_{2} \mathrm{CH}_{3}\right)$.

\section{Ethyl 2-benzoyl-3-(p-tolyl)butanoate, 20}<smiles>CCOC(=O)C(C(=O)c1ccccc1)C(C)c1ccc(C)cc1</smiles>

In a modified version of General Procedure D, 1-(p-tolyl)ethan-1-ol (871 mg, 6.4 mmol, 2 equiv.), ethyl benzoylacetate 17 (615 mg, $3.2 \mathrm{mmol}, 1$ equiv.), pentafluorophenylboronic acid 1 (34 mg, $0.16 \mathrm{mmol}$ ) and oxalic acid 2 (29 mg, $0.32 \mathrm{mmol})$ in $\mathrm{MeNO}_{2}(64 \mathrm{~mL})$ were reacted at $90{ }^{\circ} \mathrm{C}$ for $24 \mathrm{~h}$. The crude product was purified by silica-gel column chromatography (petrol/EtOAc, $98: 2$ to $\left.90: 10 \mathrm{R}_{f} .0 .22\right)$ to give $\mathbf{2 0}$ as a mixture of diastereoisomers $(54: 46 \mathrm{dr}$, $970 \mathrm{mg}, 97 \%)$ as a colourless oil. $v_{\max } 2973(\mathrm{C}-\mathrm{H}), 1733(\mathrm{C}=\mathrm{C}), 1685(\mathrm{C}=\mathrm{C}), 1515(\mathrm{C}=\mathrm{C})$;

Data for major diastereoisomer: ${ }^{1} \mathrm{H}$ NMR $\left(300 \mathrm{MHz}, \mathrm{CDCl}_{3}\right)$ (selected) $\delta_{\mathrm{H}}: 8.15-8.10(\mathrm{~m}, 2 \mathrm{H}$, $\operatorname{ArCH}), 7.66-7.60$ (m, 1H, ArCH), 7.55-7.49 (m, 2H, $\mathrm{ArCH}), 7.16-7.10$ (m, 2H, ArCH), 7.03$6.98(\mathrm{~m}, 2 \mathrm{H}, \mathrm{ArCH}), 4.64(\mathrm{~d}, J=10.7 \mathrm{~Hz}, 1 \mathrm{H}, \mathrm{ArCCOCH}), 4.32-4.18\left(\mathrm{~m}, 1 \mathrm{H}, \mathrm{CHCH}_{3}\right), 3.91-$ $3.77\left(\mathrm{~m}, 2 \mathrm{H}, \mathrm{OCH}_{2} \mathrm{CH}_{3}\right), 2.34\left(\mathrm{~s}, 3 \mathrm{H}, \mathrm{ArCCH}_{3}\right), 1.29-1.24\left(\mathrm{~m}, 3 \mathrm{H}, \mathrm{CHCH}_{3}\right), 0.93(\mathrm{t}, J=7.1$ $\left.\mathrm{Hz}, 3 \mathrm{H}, \mathrm{OCH}_{2} \mathrm{CH}_{3}\right) ;{ }^{13} \mathrm{C}\left\{{ }^{1} \mathrm{H}\right\} \mathrm{NMR}\left(126 \mathrm{MHz}, \mathrm{CDCl}_{3}\right) \delta_{\mathrm{C}}: 193.8$ (ArCCO), 168.2 (COOEt), $140.6(\mathrm{ArC}), 136.9\left(\mathrm{ArC}_{4}(4) \mathrm{CH}_{3}\right), 136.1(\mathrm{PhC}(1)), 133.8(\mathrm{PhCH}), 129.2(\mathrm{ArCH}), 128.9$ $(\mathrm{PhCH}), 128.6(\mathrm{PhCH}), 127.4(\mathrm{ArCH}), 61.3(\mathrm{ArCCOCH}), 46.2\left(\mathrm{CH}_{2} \mathrm{CH}_{3}\right), 39.4\left(\mathrm{CHCH}_{3}\right), 21.1$ $\left(\mathrm{ArCCH}_{3}\right), 20.5\left(\mathrm{CHCH}_{3}\right), 13.8\left(\mathrm{CH}_{2} \mathrm{CH}_{3}\right) ; \mathrm{HRMS}\left(\mathrm{ESI}^{+}\right) \mathrm{m} / z$ : $[\mathrm{M}+\mathrm{Na}]^{+}$calcd for $\mathrm{C}_{20} \mathrm{H}_{22} \mathrm{O}_{3} \mathrm{Na}$ 333.1461, found 333.1468. 
Data for minor diastereoisomer: ${ }^{1} \mathrm{H}$ NMR $\left(300 \mathrm{MHz}, \mathrm{CDCl}_{3}\right)$ (selected) $\delta_{\mathrm{H}}: 7.90-7.86(\mathrm{~m}, 2 \mathrm{H}$, $\operatorname{ArCH}$ ), 7.66-7.60 (m, 1H, ArCH), 7.55-7.49 (m, 2H, ArCH), 7.25-7.20 (m, 3H, ArCH), 7.16$7.10(\mathrm{~m}, 2 \mathrm{H}, \mathrm{ArCH}), 4.69$ (d, $J=10.5 \mathrm{~Hz}, 1 \mathrm{H}, \mathrm{ArCCOCH}), 4.32-4.18\left(\mathrm{~m}, 1 \mathrm{H}, \mathrm{CHCH}_{3}\right), 3.91-$ $3.77\left(\mathrm{~m}, 2 \mathrm{H}, \mathrm{CH}_{2} \mathrm{CH}_{3}\right), 2.24\left(\mathrm{~s}, 3 \mathrm{H}, \mathrm{ArCH}_{3}\right), 1.24-1.19\left(\mathrm{~m}, 3 \mathrm{H}, \mathrm{OCH}_{2} \mathrm{CH}_{3}\right) ;{ }^{13} \mathrm{C}\left\{{ }^{1} \mathrm{H}\right\} \mathrm{NMR}$ $\left(126 \mathrm{MHz}, \mathrm{CDCl}_{3}\right)$ (selected) $\delta_{\mathrm{C}}: 194.1$ (ArCCO), 168.9 (COOEt), $141.0(\mathrm{ArC}), 137.1$ $\left(\mathrm{ArC}(4) \mathrm{CH}_{3}\right), 136.4(\mathrm{ArC}(1)), 133.9(\mathrm{ArCH}), 127.7(\mathrm{ArCH}), 61.6(\mathrm{ArCCOCH}), 46.2$ $\left(\mathrm{CH}_{2} \mathrm{CH}_{3}\right), 40.0\left(\mathrm{CHCH}_{3}\right), 21.2\left(\mathrm{ArCCH}_{3}\right), 20.9\left(\mathrm{CHCH}_{3}\right), 14.2\left(\mathrm{CH}_{2} \mathrm{CH}_{3}\right)$.

\section{Ethyl 2-benzoyl-3-(4-methoxyphenyl)butanoate, 21}<smiles>CCOC(=O)C(C(=O)c1ccccc1)C(C)c1ccc(OC)cc1</smiles>

Following General Procedure D, 1-(4-methoxyphenyl)ethan-1-ol S19 (61 mg, 0.4 mmol), ethyl benzoylacetate 17 (154 mg, $0.8 \mathrm{mmol})$, pentafluorophenylboronic acid 1 (4.2 mg, 20 $\mu \mathrm{mol})$ and oxalic acid $2(3.6 \mathrm{mg}, 40 \mu \mathrm{mol})$ in $\mathrm{MeNO}_{2}(8.0 \mathrm{~mL})$ were reacted at $40{ }^{\circ} \mathrm{C}$ for $24 \mathrm{~h}$. The crude product was purified by silica-gel column chromatography (petrol/EtOAc, 95:5 to $\left.85: 15 \mathrm{R}_{f} .0 .25\right)$ to give 21 as a mixture of diastereoisomers $(53: 47 \mathrm{dr}, 110 \mathrm{mg}, 80 \%)$ as a colourless oil, with spectroscopic data in accordance with the literature. ${ }^{16}$

Data for major diastereoisomer: ${ }^{1} \mathrm{H}$ NMR (400 $\left.\mathrm{MHz} \mathrm{CDCl}_{3}\right) \delta_{\mathrm{H}}: 8.11-8.08(\mathrm{~m}, 2 \mathrm{H}$, $\operatorname{PhC}(2,6) H), 7.61-7.57$ (m, 1H, PhC(4)H), 7.51-7.46 (m, 2H, $\mathrm{PhC}(3,5) H), 7.24$ (d, J=8.7 Hz, 2H, $\operatorname{ArC}(2,6) H), 6.85(\mathrm{~d}, J=8.7 \mathrm{~Hz}, 2 \mathrm{H}, \operatorname{ArC}(3,5) H), 4.59$ (d, $J=10.6 \mathrm{~Hz}, 1 \mathrm{H}, \mathrm{C}(\mathrm{O}) \mathrm{CH})$, 3.90-3.74 (m, $3 \mathrm{H}, \mathrm{OCH}_{2}$ and $\left.\mathrm{CHCH}_{3}\right), 3.78\left(\mathrm{~s}, 3 \mathrm{H}, \mathrm{OCH}_{3}\right), 1.23\left(\mathrm{~d}, J=6.7 \mathrm{~Hz}, 3 \mathrm{H}, \mathrm{CHCH}_{3}\right)$, $0.91\left(\mathrm{t}, J=7.2 \mathrm{~Hz}, 3 \mathrm{H}, \mathrm{OCH}_{2} \mathrm{CH}_{3}\right)$.

Data for minor diastereoisomer: ${ }^{1} \mathrm{H}$ NMR $\left(400 \mathrm{MHz}, \mathrm{CDCl}_{3}\right) \delta_{\mathrm{H}}: 7.86-7.83(\mathrm{~m}, 2 \mathrm{H}$, $\mathrm{PhC}(2,6) H), 7.51-7.46$ (m, 1H, PhC(4)H), 7.38-7.34 (m, 2H, $\mathrm{PhC}(3,5) H), 7.13$ (d, J=8.7 Hz, $2 \mathrm{H}, \operatorname{ArC}(2,6) H), 6.71(\mathrm{~d}, J=8.7 \mathrm{~Hz}, 2 \mathrm{H}, \operatorname{ArC}(3,5) H), 4.64(\mathrm{~d}, J=10.4 \mathrm{~Hz}, 1 \mathrm{H}, \mathrm{C}(\mathrm{O}) \mathrm{CH}), 4.18$ $\left(\mathrm{qd}, J=7.2,1.2 \mathrm{~Hz}, 2 \mathrm{H}, \mathrm{OCH}_{2}\right), 3.90-3.74\left(\mathrm{~m}, 1 \mathrm{H}, \mathrm{CHCH}_{3}\right), 3.69\left(\mathrm{~s}, 3 \mathrm{H}, \mathrm{OCH}_{3}\right), 1.36(\mathrm{~d}, J=$ $\left.7.0 \mathrm{~Hz}, 3 \mathrm{H}, \mathrm{CHCH}_{3}\right), 1.21\left(\mathrm{t}, J=7.2 \mathrm{~Hz}, 3 \mathrm{H}, \mathrm{OCH}_{2} \mathrm{CH}_{3}\right)$. 
Ethyl 2-benzoyl-3-(4-bromophenyl)butanoate, 22<smiles>CCOC(=O)C(C(=O)c1ccccc1)C(C)c1ccc(Br)cc1</smiles>

Following General Procedure D, 1-(4-bromophenyl)ethan-1-ol S22 (80 mg, 0.4 mmol), ethyl benzoylacetate 17 (159 g, $0.8 \mathrm{mmol}$ ), pentafluorophenylboronic acid $\mathbf{1}(4.2 \mathrm{mg}, 20 \mu \mathrm{mol})$ and oxalic acid $2(3.6 \mathrm{mg}, 40 \mu \mathrm{mol})$ in $\mathrm{MeNO}_{2}(8.0 \mathrm{~mL})$ were reacted at $90{ }^{\circ} \mathrm{C}$ for $24 \mathrm{~h}$. The crude product was purified by silica-gel column chromatography (petrol/EtOAc, 95:5 to 85:15 $\mathrm{R}_{f}$. $0.19)$ to give 22 as a mixture of diastereoisomers (52:48 dr, $91 \mathrm{mg}, 61 \%)$ as a colourless oil. $v_{\max } 2979(\mathrm{C}-\mathrm{H}), 1742(\mathrm{C}=\mathrm{C}), 1695(\mathrm{C}=\mathrm{C}), 1523(\mathrm{C}=\mathrm{C})$,

Data for major diastereoisomer: ${ }^{1} \mathrm{H}$ NMR $\left(300 \mathrm{MHz}, \mathrm{CDCl}_{3}\right)$ (selected) $\delta_{\mathrm{H}}: 8.14-8.05(\mathrm{~m}, 2 \mathrm{H}$, $\mathrm{ArCH}), 7.54-7.46$ (m, 2H, ArCH), 7.46-7.36 (m, 1H, ArCH), 7.34-7.28 (m, 2H, ArCH), 7.23$7.16(\mathrm{~m}, 2 \mathrm{H}, \mathrm{ArCH}), 4.58(\mathrm{~d}, J=10.8 \mathrm{~Hz}, 1 \mathrm{H}, \mathrm{ArCCOCH}), 4.30-4.13\left(\mathrm{~m}, 1 \mathrm{H}, \mathrm{CHCH}_{3}\right), 3.90-$ $3.75\left(\mathrm{~m}, 2 \mathrm{H}, \mathrm{OCH}_{2} \mathrm{CH}_{3}\right), 1.30-1.18\left(\mathrm{~m}, 3 \mathrm{H}, \mathrm{CHCH}_{3}\right), 0.92$ (t, $\left.J=7.1 \mathrm{~Hz}, 3 \mathrm{H}, \mathrm{OCH}_{2} \mathrm{CH}_{3}\right)$; ${ }^{13} \mathrm{C}\left\{{ }^{1} \mathrm{H}\right\}$ NMR $\left(126 \mathrm{MHz}, \mathrm{CDCl}_{3}\right) \delta_{\mathrm{C}}: 193.4$ (ArCO), 168.0 (COOEt), $142.7(\operatorname{ArC}(1)), 136.2$ $(\operatorname{ArC}(1)), 133.9(\operatorname{ArC}(4) \mathrm{H}), 133.6(\operatorname{ArC}(3,5) \mathrm{H}), 129.4(\operatorname{ArC}(2,6) \mathrm{H}), 128.9(\mathrm{ArCH}), 128.7$ $(\mathrm{ArCH}), 120.4(\mathrm{ArC}(4) \mathrm{Br}), 61.4(\mathrm{ArCCOCH}), 46.2\left(\mathrm{CH}_{2} \mathrm{CH}_{3}\right), 39.3\left(\mathrm{CHCH}_{3}\right), 20.3\left(\mathrm{CHCH}_{3}\right)$, $13.9\left(\mathrm{CH}_{2} \mathrm{CH}_{3}\right)$; HRMS (ESI $\left.{ }^{-}\right) \mathrm{m} / z$ : $[\mathrm{M}-\mathrm{H}]^{-}$calcd for $\mathrm{C}_{19} \mathrm{H}_{18} \mathrm{BrO}_{3} 373.0445$, found 373.0446 .

Data for minor diastereoisomer: ${ }^{1} \mathrm{H}$ NMR $\left(300 \mathrm{MHz}, \mathrm{CDCl}_{3}\right)$ (selected) $\delta_{\mathrm{H}}: 7.89-7.82(\mathrm{~m}, 2 \mathrm{H}$, $\mathrm{ArCH}), 7.66-7.57$ (m, 1H, ArCH), 7.54-7.47 (m, 2H, ArCH), 7.46-7.36 (m, 2H, ArCH), 7.14$7.06(\mathrm{~m}, 2 \mathrm{H}, \mathrm{ArCH}), 4.62(\mathrm{~d}, J=10.7 \mathrm{~Hz}, 1 \mathrm{H}, \mathrm{ArCCOCH}), 4.30-4.13\left(\mathrm{~m}, 1 \mathrm{H}, \mathrm{CHCH}_{3}\right), 3.90-$ $3.75\left(\mathrm{~m}, 2 \mathrm{H}, \mathrm{OCH}_{2} \mathrm{CH}_{3}\right), 1.30-1.18\left(\mathrm{~m}, 3 \mathrm{H}, \mathrm{OCH}_{2} \mathrm{CH}_{3}\right),{ }^{13} \mathrm{C}\left\{{ }^{1} \mathrm{H}\right\} \mathrm{NMR}\left(126 \mathrm{MHz}, \mathrm{CDCl}_{3}\right)$ (selected) $\delta_{\mathrm{C}}: 193.6$ (ArCO), 168.5 (COOEt), 143.1 (ArC(1)), 136.9 (ArC(1)), 133.9 $(\mathrm{ArC}(3,5) \mathrm{H}), 129.6(\mathrm{ArC}(2,6) \mathrm{H}), 129.0(\mathrm{ArCH}), 128.8(\mathrm{ArCH}), 120.7(\mathrm{ArC}(4) \mathrm{Br}), 61.6$ (ArCCOCH), $39.4\left(\mathrm{CHCH}_{3}\right), 20.7\left(\mathrm{CHCH}_{3}\right), 14.2\left(\mathrm{CH}_{2} \mathrm{CH}_{3}\right)$. 


\section{Decarboxylation of 1,3-Ketoesters}

General Procedure E: Decarboxylation with $\mathrm{KOH}$

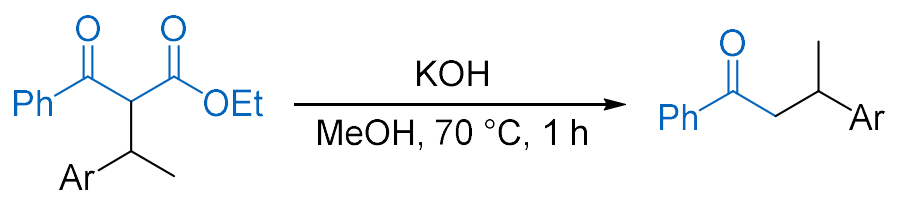

In accordance with the literature, ${ }^{20}$ the required ethyl 3-aryl-2-benzoylbutanoate (1.0 equiv.) was dissolved in $\mathrm{MeOH}(0.5 \mathrm{M})$ before aqueous $\mathrm{KOH}\left(10\right.$ equiv., $4.4 \mathrm{M}$ in $\mathrm{H}_{2} \mathrm{O}$ ) was added. The reaction was heated at $70{ }^{\circ} \mathrm{C}$ until complete by TLC analysis $(c a .1 \mathrm{~h})$. The reaction was cooled to $\mathrm{rt}$ before $\mathrm{CH}_{2} \mathrm{Cl}_{2}(20 \mathrm{~mL})$ was added. The solution was washed with water, brine, dried over $\mathrm{MgSO}_{4}$, filtered, and concentrate under reduced pressure. The crude product was purified by silica-gel column chromatography (Petrol/EtOAc).

General Procedure F: Decarboxylation with $\mathrm{NaOH}$

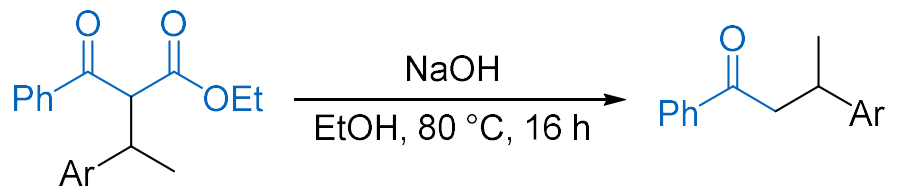

In accordance with the literature, ${ }^{21}$ the required ethyl 3-aryl-2-benzoylbutanoate (1.0 equiv.) was dissolved in $\mathrm{EtOH}(0.75 \mathrm{M})$ before aqueous $\mathrm{NaOH}\left(47\right.$ equiv., $2 \mathrm{M}$ in $\mathrm{H}_{2} \mathrm{O}$ ) was added. The reaction was heated at $80{ }^{\circ} \mathrm{C}$ for $16 \mathrm{~h}$. The reaction was cooled to $\mathrm{rt}$ before being acifdifid to $\mathrm{pH} 4$ using $2 \mathrm{M} \mathrm{HCl}$. The solution was extracted with $\mathrm{Et}_{2} \mathrm{O}(3 \times 20 \mathrm{~mL})$, washed with water, brine, dried over $\mathrm{MgSO}_{4}$, filtered, and concentrated under reduced pressure. The crude product was purified by silica-gel column chromatography (Petrol/EtOAc).

\section{1,3-Diphenylbutan-1-one, 23}<smiles>CC(CC(=O)c1ccccc1)c1ccccc1</smiles>

Following General Procedure E, ethyl 2-benzoyl-3-phenylbutanoate 19 (50 mg, $0.17 \mathrm{mmol}$ ) in $\mathrm{MeOH}(0.5 \mathrm{~mL})$, was added $\mathrm{KOH}\left(100 \mathrm{mg}, 1.7 \mathrm{mmol}\right.$ in $0.5 \mathrm{~mL}$ of $\left.\mathrm{H}_{2} \mathrm{O}\right)$ and was heated at reflux for $1 \mathrm{~h}$. The crude product was purified by silica-gel column chromatography (petrol/EtOAc, 98:2 to 90:10 $\mathrm{R}_{f}$ : 0.31) to give title compound $\mathbf{2 3}(19 \mathrm{mg}, 49 \%)$ as a colourless oil, with spectroscopic data in accordance with the literature. $.^{22} \mathrm{mp} 67-69^{\circ} \mathrm{C}\left\{\right.$ Lit. $\left.^{22} 68-69^{\circ} \mathrm{C}\right\}$; ${ }^{1} \mathrm{H}$ NMR (300 MHz, $\left.\mathrm{CDCl}_{3}\right) \delta_{\mathrm{H}}$ : 7.99-7.87 (m, 2H, $\left.\mathrm{ArCH}\right), 7.61-7.49$ (m, 1H, ArCH), 7.497.39 (m, 2H, ArCH), 7.36-7.26 (m, 4H, ArCH), 7.23-7.15 (m, 1H, ArCH), 3.57-3.43 (m, 1H, $\left.\mathrm{CHCH}_{3}\right), 3.31\left(\mathrm{dd}, J=16.5,5.7 \mathrm{~Hz}, 1 \mathrm{H}, \mathrm{CH}^{\mathrm{A}} H^{B}\right), 3.19\left(\mathrm{dd}, J=16.5,8.3 \mathrm{~Hz}, 1 \mathrm{H}, \mathrm{CH}^{A} \mathrm{H}^{\mathrm{B}}\right)$, 
$1.34\left(\mathrm{~d}, J=6.9 \mathrm{~Hz}, 3 \mathrm{H}, \mathrm{CHCH}_{3}\right)$.

1-Phenyl-3-(p-tolyl)butan-1-one, 24<smiles>Cc1ccc(C(C)CC(=O)c2ccccc2)cc1</smiles>

Following General Procedure F, ethyl 2-benzoyl-3-(p-tolyl)butanoate 20 (266 mg, 0.85 $\mathrm{mmol})$, aqueous $2 \mathrm{M} \mathrm{NaOH}(4 \mathrm{~mL})$ in $\mathrm{EtOH}(4 \mathrm{~mL})$ was heated at reflux for $16 \mathrm{~h}$. Crude product was purified by silica-gel column chromatography (petrol/EtOAc, 98:2 to 95:5 $+\mathrm{Et}_{3} \mathrm{~N}$ (1\%), $\mathrm{R}_{f}$. 0.27) to give title compound $\mathbf{2 4}(198 \mathrm{~g}, 98 \%)$ as a viscous yellow oil, with spectroscopic data in accordance with the literature. ${ }^{22}{ }^{1} \mathrm{H}$ NMR $\left(300 \mathrm{MHz}, \mathrm{CDCl}_{3}\right) \delta_{\mathrm{H}}: 7.98-7.90(\mathrm{~m}, 2 \mathrm{H}$, $\operatorname{ArCH}$ ), 7.60-7.51 (m, 1H, ArCH), 7.50-7.38 (m, 2H, ArCH), 7.21-7.07 (m, 4H, ArCH), 3.55$3.39\left(\mathrm{~m}, 1 \mathrm{H}, \mathrm{CHCH}_{3}\right), 3.28\left(\mathrm{dd}, J=16.5,5.7 \mathrm{~Hz}, 1 \mathrm{H}, \mathrm{CH}^{\mathrm{A}} H^{B}\right), 3.17(\mathrm{dd}, J=16.5,8.3 \mathrm{~Hz}, 1 \mathrm{H}$, $\left.\mathrm{CH}^{A} \mathrm{H}^{\mathrm{B}}\right), 2.32\left(\mathrm{~s}, 3 \mathrm{H}, \mathrm{ArCCH}_{3}\right), 1.32\left(\mathrm{~d}, J=6.9 \mathrm{~Hz}, 3 \mathrm{H}, \mathrm{CHCH}_{3}\right)$.

\section{3-(4-Methoxyphenyl)-1-phenylbutan-1-one, 25}

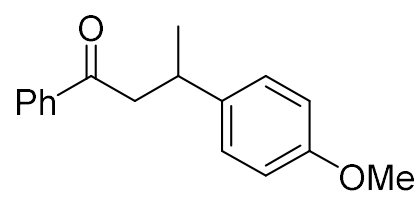

Following General Procedure F ethyl 2-benzoyl-3-(4-methoxy)butanoate 21 (200 mg, 0.60 mmol), aqueous $2 \mathrm{M} \mathrm{NaOH}(4 \mathrm{~mL})$ in $\mathrm{EtOH}(4 \mathrm{~mL})$ was heated at reflux for $16 \mathrm{~h}$. The crude product was purified by silica-gel column chromatography (petrol/EtOAc, 98:2 to 95:5 $\mathrm{R}_{f}$. $0.23)$ to give title compound 25 (99 $\mathrm{mg}, 65 \%$ ) as a white powder, with spectroscopic data in accordance with the literature. ${ }^{22} \mathrm{mp} 66-68{ }^{\circ} \mathrm{C}\left\{\right.$ Lit. $\left.^{22} 64-66{ }^{\circ} \mathrm{C}\right\} ;{ }^{1} \mathrm{H} \mathrm{NMR}\left(300 \mathrm{MHz}, \mathrm{CDCl}_{3}\right)$ $\delta_{\mathrm{H}}$ : 7.98-7.88 (m, 2H, ArCH), 7.62-7.51 (m, 1H, ArCH), 7.50-7.40 (m, 2H, ArCH), 7.24-7.13 $(\mathrm{m}, 2 \mathrm{H}, \operatorname{ArC}(2,6) H), 6.91-6.78(\mathrm{~m}, 2 \mathrm{H}, \operatorname{ArC}(3,5) H), 3.79\left(\mathrm{~s}, 3 \mathrm{H}, \mathrm{OCH}_{3}\right), 3.53-3.38(\mathrm{~m}, 1 \mathrm{H}$, $\left.\mathrm{CHCH}_{3}\right), 3.27\left(\mathrm{dd}, J=16.4,6.8 \mathrm{~Hz}, 1 \mathrm{H}, \mathrm{CH}^{A} H^{\mathrm{B}}\right), 3.15\left(\mathrm{dd}, J=16.4,8.1 \mathrm{~Hz}, 1 \mathrm{H}, \mathrm{CH}^{A} \mathrm{H}^{\mathrm{B}}\right), 1.31$ $\left(\mathrm{d}, J=6.9 \mathrm{~Hz}, 3 \mathrm{H}, \mathrm{CHCH}_{3}\right)$. 


\section{Dehydrative Allylation}

General Procedure G: Dehydrative allylation

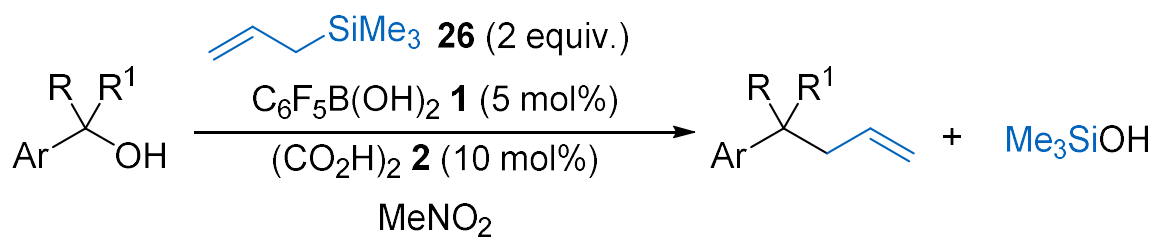

Allyltrimethylsilane 26 ( 2 equiv.) was added to a solution of pentafluorophenylboronic acid 1 $(5 \mathrm{~mol} \%)$ and oxalic acid $2(10 \mathrm{~mol} \%)$ in $\mathrm{MeNO}_{2}(0.05 \mathrm{M})$ and was stirred at $\mathrm{rt}$ for $5 \mathrm{mins}$. The required benzylic alcohol (1.0 equiv.) was added and the reaction stirred at the stated temperature until complete by TLC analysis. The reaction was cooled to rt, diluted with toluene and concentrated under reduced pressure to give the crude product, which was further purified by silica-gel column chromatography.

\section{But-3-ene-1,1-diyldibenzene, 27}<smiles>C=CCC(c1ccccc1)c1ccccc1</smiles>

Following a modified General Procedure G, benzhydrol (74 mg, $0.4 \mathrm{mmol}$ ), allyltrimethylsilane 26 (320 $\mu \mathrm{L}, 2$ mmol, 5 equiv.), pentafluorophenylboronic acid $\mathbf{1}(4.2 \mathrm{mg}$, $20 \mu \mathrm{mol})$ and oxalic acid $2(3.6 \mathrm{mg}, 40 \mu \mathrm{mol})$ in $\mathrm{MeNO}_{2}(8.0 \mathrm{~mL})$ were reacted at $90{ }^{\circ} \mathrm{C}$ for $16 \mathrm{~h}$. The crude product was purified by silica-gel column chromatography (petrol/EtOAc, 95:5 $\left.\mathrm{R}_{f} .0 .33\right)$ to give title compound $27(80 \mathrm{mg}, 96 \%)$ as a colourless oil, with spectroscopic data in accordance with the literature. ${ }^{23}{ }^{1} \mathrm{H}$ NMR $\left(300 \mathrm{MHz}, \mathrm{CDCl}_{3}\right) \delta_{\mathrm{H}}: 7.36-7.15(\mathrm{~m}, 10 \mathrm{H}$, $\mathrm{ArCH}+2), 5.84-5.66\left(\mathrm{~m}, 1 \mathrm{H}, \mathrm{CH}_{2} \mathrm{CHCH}_{2}\right), 5.12-4.94\left(\mathrm{~m}, 2 \mathrm{H}, \mathrm{CH}_{2} \mathrm{CHCH}_{2}\right), 4.04(\mathrm{t}, J=7.9$ $\mathrm{Hz}, 1 \mathrm{H}, \mathrm{ArCCH}), 2.90-2.79\left(\mathrm{~m}, 2 \mathrm{H}, \mathrm{CH}_{2} \mathrm{CH}\right)$.

\section{1-Methyl-4-(pent-4-en-2-yl)benzene, 28}

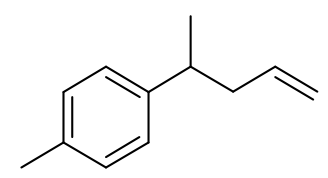

Following General Procedure G, 1-(p-tolyl)ethan-1-ol $(54 \quad \mathrm{mg}, \quad 0.4 \mathrm{mmol})$, allyltrimethylsilane $26(130 \mu \mathrm{L}, 0.8 \mathrm{mmol})$, pentafluorophenylboronic acid 1 (4.2 $\mathrm{mg}, 20$ $\mu \mathrm{mol})$ and oxalic acid $2(3.6 \mathrm{mg}, 40 \mu \mathrm{mol})$ in $\mathrm{MeNO}_{2}(8.0 \mathrm{~mL})$ were reacted at $\mathrm{rt}$ for $16 \mathrm{~h}$. The crude product was purified by silica-gel column chromatography (petrol/EtOAc, 98:2 Rf: 0.31) to give title compound $\mathbf{2 8}(37 \mathrm{mg}, 40 \%)$ as a colourless oil, with spectroscopic data in 
accordance with the literature. ${ }^{24}{ }^{1} \mathrm{H}$ NMR $\left(300 \mathrm{MHz}, \mathrm{CDCl}_{3}\right) \delta_{\mathrm{H}}: 7.13-7.07(\mathrm{~m}, 4 \mathrm{H}, \mathrm{ArCH})$, 5.78-5.65 (m, 1H, $\left.\mathrm{CH}_{2} \mathrm{CHCH}_{2}\right), 5.03-4.93\left(\mathrm{~m}, 2 \mathrm{H}, \mathrm{CH}_{2} \mathrm{CHCH}_{2}\right), 2.81-2.70\left(\mathrm{~m}, 1 \mathrm{H}, \mathrm{CH}_{2} \mathrm{CH}\right)$, $2.32\left(\mathrm{~s}, 3 \mathrm{H}, \mathrm{ArCH}_{3}\right), 2.42-2.21\left(\mathrm{~m}, 2 \mathrm{H}, \mathrm{CH}_{2} \mathrm{CH}\right), 1.23\left(\mathrm{~d}, J=6.9 \mathrm{~Hz}, 3 \mathrm{H}, \mathrm{CHCH}_{3}\right)$.

1-Methoxy-4-(pent-4-en-2-yl)benzene, 29<smiles>C=CCC(C)c1ccc(OC)cc1</smiles>

Following General Procedure G, 1-(4-methoxyphenyl)ethan-1-ol S19 (61 mg, 0.4 mmol), allyltrimethylsilane $26(130 \mu \mathrm{L}, 0.8 \mathrm{mmol})$, pentafluorophenylboronic acid 1 (4.2 mg, 20 $\mu \mathrm{mol})$ and oxalic acid $2(3.6 \mathrm{mg}, 40 \mu \mathrm{mol})$ in $\mathrm{MeNO}_{2}(8.0 \mathrm{~mL})$ were reacted at $\mathrm{rt}$ for $16 \mathrm{~h}$. The crude product was purified by silica-gel column chromatography (petrol/EtOAc, 98:2 $\mathrm{R}_{f}$. 0.38) to give title compound 29 (54 mg, 76\%) as a colourless oil, with spectroscopic data in accordance with the literature. ${ }^{24}{ }^{1} \mathrm{H}$ NMR $\left(300 \mathrm{MHz}, \mathrm{CDCl}_{3}\right) \delta_{\mathrm{H}}: 7.17-7.05(\mathrm{~m}, 2 \mathrm{H}$, $\operatorname{ArC}(2,6) H), 6.90-6.77(\mathrm{~m}, 2 \mathrm{H}, \operatorname{ArC}(3,5) H), 5.80-5.60\left(\mathrm{~m}, 1 \mathrm{H}, \mathrm{CH}_{2} \mathrm{CHCH}_{2}\right), 5.06-4.89$ (m, $\left.2 \mathrm{H}, \mathrm{CH}_{2} \mathrm{CHCH}_{2}\right), 3.79\left(\mathrm{~s}, 3 \mathrm{H}, \mathrm{OCH}_{3}\right), 2.84-2.67\left(\mathrm{~m}, 1 \mathrm{H}, \mathrm{CH}_{2} \mathrm{CH}\right), 2.42-2.15(\mathrm{~m}, 2 \mathrm{H}$, $\left.\mathrm{CH}_{2} \mathrm{CH}\right), 1.23\left(\mathrm{~d}, \mathrm{~J}=7.0 \mathrm{~Hz}, 3 \mathrm{H}, \mathrm{CHCH}_{3}\right)$.

\section{1-Fluoro-4-(pent-4-en-2-yl)benzene, 30}

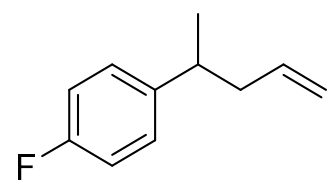

Following General Procedure G, 1-(4-fluorophenyl)ethan-1-ol S21 (56 mg, $0.4 \mathrm{mmol}$ ), allyltrimethylsilane 26 (130 $\mu 1,0.8 \mathrm{mmol}$ ), pentafluorophenylboronic acid $\mathbf{1}$ (4.2 $\mathrm{mg}, 20 \mu \mathrm{mol}$ ) and oxalic acid $2(3.6 \mathrm{mg}, 40 \mu \mathrm{mol})$ in $\mathrm{MeNO}_{2}(8.0 \mathrm{~mL})$ were reacted at $\mathrm{rt}$ for $16 \mathrm{~h}$. The crude product was either extracted with EtOAc $(3 \times 10 \mathrm{~mL})$, washed with $\mathrm{NaHCO}_{3}$, brine, dried over anhydrous $\mathrm{MgSO}_{4}$, filtered, and concentrated under reduced pressure. The crude material was purified by silica-gel column chromatography (petrol/EtOAc, 98:2 to 95:5 $\mathrm{R}_{f} .0 .42$ ) to give title compound 30 (35 mg, 55\%) as a colourless oil, with spectroscopic data in accordance with the literature. ${ }^{24}{ }^{1} \mathrm{H}$ NMR $\left(300 \mathrm{MHz}, \mathrm{CDCl}_{3}\right) \delta_{\mathrm{H}}: 7.19-7.09$ (m, 2H, $\left.\operatorname{ArC}(2,6) H\right), 7.04-6.92(\mathrm{~m}, 2 \mathrm{H}$, $\operatorname{ArC}(3,5) H), 5.77-5.59\left(\mathrm{~m}, 1 \mathrm{H}, \mathrm{CH}_{2} \mathrm{CHCH}_{2}\right), 5.04-4.90\left(\mathrm{~m}, 2 \mathrm{H}, \mathrm{CH}_{2} \mathrm{CHCH}_{2}\right), 2.86-2.70(\mathrm{~m}$, $\left.1 \mathrm{H}, \mathrm{CHCH}_{3}\right), 2.40-2.20\left(\mathrm{~m}, 2 \mathrm{H}, \mathrm{CHCH}_{2} \mathrm{CH}\right), 1.23\left(\mathrm{~d}, \mathrm{~J}=6.9 \mathrm{~Hz}, 3 \mathrm{H}, \mathrm{CHCH}_{3}\right)$. 


\section{1-Bromo-4-(pent-4-en-2-yl)benzene, 31}<smiles>C=CCC(C)c1ccc(Br)cc1</smiles>

Following General Procedure G, 1-(4-bromophenyl)ethan-1-ol S22 (80 mg, 0.4 mmol), allyltrimethylsilane $26(130 \mu \mathrm{L}, 0.8 \mathrm{mmol})$, pentafluorophenylboronic acid 1 (4.2 $\mathrm{mg}, 20$ $\mu \mathrm{mol})$ and oxalic acid $2(3.6 \mathrm{mg}, 40 \mu \mathrm{mol})$ in $\mathrm{MeNO}_{2}(8.0 \mathrm{~mL})$ were reacted at $\mathrm{rt}$ for $16 \mathrm{~h}$. The crude product was purified by silica-gel column chromatography (petrol/EtOAc, 90:10 $\mathrm{R}_{f}$ : $0.36)$ to give title compound $31(51 \mathrm{mg}, 43 \%)$ as a colourless oil, with spectroscopic data in accordance with the literature. ${ }^{24}{ }^{1} \mathrm{H}$ NMR $\left(300 \mathrm{MHz}, \mathrm{CDCl}_{3}\right) \delta_{\mathrm{H}}: 7.49-7.32(\mathrm{~m}, 2 \mathrm{H}$, $\operatorname{ArC}(3,5) H), 7.14-6.96(\mathrm{~m}, 2 \mathrm{H}, \operatorname{ArC}(2,6) H), 5.80-5.60\left(\mathrm{~m}, 1 \mathrm{H}, \mathrm{CH}_{2} \mathrm{CHCH}_{2}\right), 5.04-4.90$ (m, $2 \mathrm{H}, \mathrm{CH}_{2} \mathrm{CHCH}_{2}$ ), 2.84-2.67 (m, $\left.1 \mathrm{H}, \mathrm{CH}_{2} \mathrm{CH}\right), 2.40-2.16\left(\mathrm{~m}, 2 \mathrm{H}, \mathrm{CH}_{2} \mathrm{CH}\right), 1.22$ (d, J= 7.0 $\left.\mathrm{Hz}, 3 \mathrm{H}, \mathrm{CHCH}_{3}\right)$.

\section{1-Iodo-3-(pent-4-en-2-yl)benzene, 32}<smiles>C=CCC(C)c1cccc(I)c1</smiles>

Following General Procedure G, 1-(3-iodophenyl)ethan-1-ol S28 (100 mg, 0.4 mmol), allyltrimethylsilane $26(130 \mu \mathrm{L}, 0.8 \mathrm{mmol})$, pentafluorophenylboronic acid 1 (4.2 $\mathrm{mg}, 20$ $\mu \mathrm{mol})$ and oxalic acid $2(3.6 \mathrm{mg}, 40 \mu \mathrm{mol})$ in $\mathrm{MeNO}_{2}(8.0 \mathrm{~mL})$ were reacted at $90^{\circ} \mathrm{C}$ for $16 \mathrm{~h}$. The crude product was purified by silica-gel column chromatography (petrol/EtOAc, 95:5 $\mathrm{R}_{f}$. $0.33)$ to give title compound 32 (45 mg, 41\%) as a colourless oil. $v_{\max } 3074(\mathrm{C}-\mathrm{H}), 2961$ (C$\mathrm{H}), 1639(\mathrm{C}=\mathrm{C}), 1561(\mathrm{C}=\mathrm{C}) ;{ }^{1} \mathrm{H}$ NMR $\left(300 \mathrm{MHz}, \mathrm{CDCl}_{3}\right) \delta_{\mathrm{H}}: 7.56-7.48(\mathrm{~m}, 2 \mathrm{H}, \mathrm{ArCH})$, 7.19-7.12 (m, 1H, $\mathrm{ArCH}$ ), 7.07-6.97 (m, 1H, $\mathrm{ArCH}), 5.77-5.58\left(\mathrm{~m}, 1 \mathrm{H}, \mathrm{CH}_{2} \mathrm{CHCH}_{2}\right), 5.05-$ $4.91\left(\mathrm{~m}, 2 \mathrm{H}, \mathrm{CH}_{2} \mathrm{CHCH}_{2}\right), 2.79-2.63\left(\mathrm{~m}, 1 \mathrm{H}, \mathrm{CH}_{2} \mathrm{CH}\right), 2.43-2.17\left(\mathrm{~m}, 2 \mathrm{H}, \mathrm{CH}_{2} \mathrm{CH}\right), 1.22$ (d, $\left.J=6.9 \mathrm{~Hz}, 3 \mathrm{H}, \mathrm{CHCH}_{3}\right) ;{ }^{13} \mathrm{C}\left\{{ }^{1} \mathrm{H}\right\} \mathrm{NMR}\left(126 \mathrm{MHz}, \mathrm{CDCl}_{3}\right) \delta_{\mathrm{C}}: 149.7(\mathrm{ArC}(1) \mathrm{I}), 136.7$ $(\mathrm{ArC}(2) \mathrm{I}), 136.3\left(\mathrm{CHCH}_{2}\right), 135.2(\mathrm{ArC}(4) \mathrm{I}), 130.2(\mathrm{ArC}(5) \mathrm{I}), 126.5(\mathrm{ArC}(6) \mathrm{I}), 116.5$ $\left(\mathrm{CHCH}_{2}\right), 94.6(\mathrm{ArC}(3) \mathrm{I}), 42.6\left(\mathrm{CHCH}_{2} \mathrm{CH}\right), 39.6\left(\mathrm{CHCH}_{3}\right), 21.2\left(\mathrm{CHCH}_{3}\right) ; \mathrm{HRMS}_{\left(\mathrm{ESI}^{-}\right)}$ $m / z:[\mathrm{M}]^{-}$calcd for $\mathrm{C}_{11} \mathrm{H}_{13} \mathrm{I} 272.0062$, found 272.0052 . 


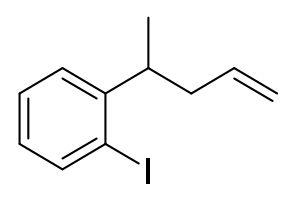

Following General Procedure G, 1-(2-iodophenyl)ethan-1-ol S29 (100 mg, 0.4 mmol), allyltrimethylsilane 26 (130 $\mu 1,0.8 \mathrm{mmol})$, pentafluorophenylboronic acid $\mathbf{1}$ (4.2 $\mathrm{mg}, 20 \mu \mathrm{mol})$ and oxalic acid $2(3.6 \mathrm{mg}, 40 \mu \mathrm{mol})$ in $\mathrm{MeNO}_{2}(8.0 \mathrm{~mL})$ were reacted at $90{ }^{\circ} \mathrm{C}$ for $16 \mathrm{~h}$. Crude product was purified by silica-gel column chromatography (petrol/EtOAc, 95:5 $\mathrm{R}_{f}: 0.34$ ) to give title compound 33 (59 mg, 54\%) as a colourless oil. $v_{\max } 3084(\mathrm{C}-\mathrm{H}), 2943(\mathrm{C}-\mathrm{H}), 1628$ $(\mathrm{C}=\mathrm{C}), 1542(\mathrm{C}=\mathrm{C}) ;{ }^{1} \mathrm{H}$ NMR $\left(300 \mathrm{MHz}, \mathrm{CDCl}_{3}\right) \delta_{\mathrm{H}}: 7.86-7.78(\mathrm{~m}, 1 \mathrm{H}, \mathrm{ArCH}), 7.35-7.26(\mathrm{~m}$, $1 \mathrm{H}, \operatorname{ArCH}), 7.21-7.14(\mathrm{~m}, 1 \mathrm{H}, \mathrm{ArCH}), 6.94-6.83(\mathrm{~m}, 1 \mathrm{H}, \mathrm{ArCH}), 5.85-5.64(\mathrm{~m}, 1 \mathrm{H}$, $\mathrm{CH}_{2} \mathrm{CHCH}_{2}$ ), 5.07-4.93 (m, 2H, $\left.\mathrm{CH}_{2} \mathrm{CHCH}_{2}\right), 3.23-3.07$ (m, $\left.1 \mathrm{H}, \mathrm{CH}_{2} \mathrm{CH}\right), 2.47-2.33(\mathrm{~m}, 1 \mathrm{H}$, $\left.\mathrm{CH}^{\mathrm{A}} \mathrm{CH}^{B} \mathrm{CH}\right), 2.29-2.13\left(\mathrm{~m}, 1 \mathrm{H}, \mathrm{CH}^{A} \mathrm{CH}^{\mathrm{B}} \mathrm{CH}\right), 1.21\left(\mathrm{~d}, J=6.9 \mathrm{~Hz}, 3 \mathrm{H}, \mathrm{CHCH}_{3}\right) ;{ }^{13} \mathrm{C}\left\{{ }^{1} \mathrm{H}\right\}$ NMR (126 MHz, $\left.\mathrm{CDCl}_{3}\right) \delta_{\mathrm{C}}: 148.8(\mathrm{ArC}(1) \mathrm{H}), 139.7(\mathrm{ArC}(3) \mathrm{H}), 136.6\left(\mathrm{CHCH}_{2}\right), 128.6$ $(\mathrm{ArC}(6) \mathrm{H}), 127.9(\mathrm{ArC}(4) \mathrm{H}), 126.8(\mathrm{ArC}(5) \mathrm{H}), 116.5\left(\mathrm{CHCH}_{2}\right), 101.7(\mathrm{ArC}(1) \mathrm{I}), 43.1$ $\left(\mathrm{CHCH}_{3}\right), 41.7\left(\mathrm{CHCH}_{2} \mathrm{CH}\right), 20.6\left(\mathrm{CHCH}_{3}\right)$; HRMS $\left(\mathrm{ESI}^{-}\right) \mathrm{m} / z$ : [M-H] $]^{-}$calcd for $\mathrm{C}_{10} \mathrm{H}_{13} \mathrm{I}$ 271.0052 , found 271.0042 .

\section{1-(2,2-Dimethylhex-5-en-3-yl)-4-methoxybenzene, 34}<smiles>C=CCC(c1ccc(OC)cc1)C(C)(C)C</smiles>

Following General Procedure G, 1-(4-methoxyphenyl)-2,2-dimethylpropan-1-ol S30 (40 mg, $0.2 \mathrm{mmol})$, allyltrimethylsilane $26(64 \mu \mathrm{L}, 0.4 \mathrm{mmol})$, pentafluorophenylboronic acid 1 (2 $\mathrm{mg}$, $10 \mu \mathrm{mol})$ and oxalic acid $2(1 \mathrm{mg}, 20 \mu \mathrm{mol})$ in $\mathrm{MeNO}_{2}(4.0 \mathrm{~mL})$ were reacted at $\mathrm{rt}$ for $16 \mathrm{~h}$. The crude product was purified by silica-gel column chromatography (petrol/EtOAc, 95:5 to 90:10 $\left.\mathrm{R}_{f} .0 .31\right)$ to give title compound $34(38 \mathrm{mg}, 86 \%)$ as a colourless oil. $v_{\max } 3030(\mathrm{C}-\mathrm{H})$, $2931(\mathrm{C}-\mathrm{H}), 1698(\mathrm{C}=\mathrm{C}), 1581(\mathrm{C}=\mathrm{C}) ;{ }^{1} \mathrm{H}$ NMR $\left(300 \mathrm{MHz}, \mathrm{CDCl}_{3}\right) \delta_{\mathrm{H}}: 7.05-7.03(\mathrm{~m}, 2 \mathrm{H}$, $\operatorname{ArC}(2,6) H), 6.82-6.80(\mathrm{~m}, 2 \mathrm{H}, \operatorname{ArC}(3,5) H), 5.55-5.47\left(\mathrm{~m}, 1 \mathrm{H}, \mathrm{CH}_{2} \mathrm{CHCH}_{2}\right), 4.93-4.78(\mathrm{~m}$, $\left.2 \mathrm{H}, \mathrm{CH}_{2} \mathrm{CHCH}_{2}\right), 3.79\left(\mathrm{~s}, 3 \mathrm{H}, \mathrm{OCH}_{3}\right), 2.56-2.51\left(\mathrm{~m}, 1 \mathrm{H}, \mathrm{CH}_{2} \mathrm{CH}\right), 2.45-2.36(\mathrm{~m}, 2 \mathrm{H}$, $\left.\mathrm{CH}_{2} \mathrm{CH}\right), 0.87\left(\mathrm{~s}, 9 \mathrm{H}, \mathrm{C}\left(\mathrm{CH}_{3}\right)_{3}\right) ;{ }^{13} \mathrm{C}\left\{{ }^{1} \mathrm{H}\right\} \mathrm{NMR}\left(126 \mathrm{MHz}, \mathrm{CDCl}_{3}\right) \delta_{\mathrm{C}}: 157.9$ (ArC(4)OMe), $138.7\left(\mathrm{CHCH}_{2}\right), 134.5(\mathrm{ArC}(1)), 130.6(\mathrm{ArC}(2,6) \mathrm{H}), 115.1\left(\mathrm{CHCH}_{2}\right), 113.0(\operatorname{ArC}(3,5) \mathrm{H}), 56.0$

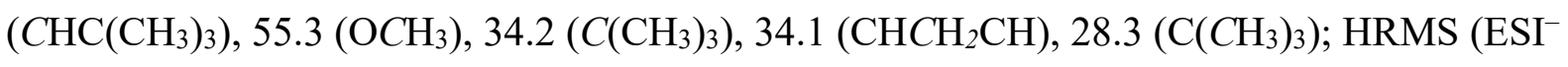
) $m / z:[\mathrm{M}]^{-}$calcd for $\mathrm{C}_{15} \mathrm{H}_{22} \mathrm{O} 218.1671$, found 218.1650 . 


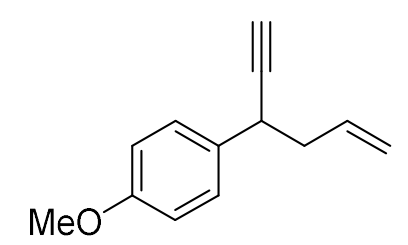

Following General Procedure G, 1-(4-methoxyphenyl)prop-2-yn-1-ol S25 (32 mg, 0.2 mmol), allyltrimethylsilane 26 (64 $\mu \mathrm{L}, 0.4 \mathrm{mmol})$, pentafluorophenylboronic acid $\mathbf{1}(2 \mathrm{mg}, 10$ $\mu \mathrm{mol})$ and oxalic acid $2(1 \mathrm{mg}, 20 \mu \mathrm{mol})$ in $\mathrm{MeNO}_{2}(4.0 \mathrm{~mL})$ were reacted at $\mathrm{rt}$ for $16 \mathrm{~h}$. The crude product was purified by silica-gel column chromatography (petrol/EtOAc, 95:5 to 90:10 $\left.\mathrm{R}_{f} .0 .31\right)$ to give title compound 35 (32 $\mathrm{mg}, 86 \%$ ) as a colourless oil, with spectroscopic data in accordance with the literature. ${ }^{25}{ }^{1} \mathrm{H}$ NMR $\left(300 \mathrm{MHz}, \mathrm{CDCl}_{3}\right) \delta_{\mathrm{H}}: 7.30-7.24(\mathrm{~m}, 2 \mathrm{H}$, $\operatorname{ArC}(2,6) H), 6.92-6.82(\mathrm{~m}, 2 \mathrm{H}, \operatorname{ArC}(3,5) H), 5.94-5.75\left(\mathrm{~m}, 1 \mathrm{H}, \mathrm{CH}_{2} \mathrm{CHCH}_{2}\right), 5.15-4.99$ (m, $\left.2 \mathrm{H}, \mathrm{CH}_{2} \mathrm{CHCH}_{2}\right), 3.80\left(\mathrm{~s}, 3 \mathrm{H}, \mathrm{OCH}_{3}\right), 3.71-3.60\left(\mathrm{~m}, 1 \mathrm{H}, \mathrm{CH}_{2} \mathrm{CH}\right), 2.54-2.44(\mathrm{~m}, 2 \mathrm{H}$, $\left.\mathrm{CH}_{2} \mathrm{CH}\right), 2.29(\mathrm{~d}, J=2.5 \mathrm{~Hz}, 1 \mathrm{H}, \mathrm{CCH})$.

\section{1-(Hepta-1,6-dien-4-yl)-4-methoxybenzene, 36}

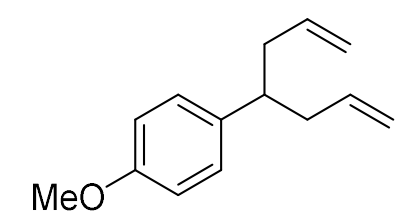

Following General Procedure G, 1-(4-methoxyphenyl)but-3-en-1-ol (36 mg, 0.2 mmol), allyltrimethylsilane $26(64 \mu \mathrm{L}, 0.4 \mathrm{mmol})$, pentafluorophenylboronic acid $\mathbf{1}(2 \mathrm{mg}, 10 \mu \mathrm{mol})$ and oxalic acid $2(1 \mathrm{mg}, 20 \mu \mathrm{mol})$ in $\mathrm{MeNO}_{2}(4.0 \mathrm{~mL})$ were reacted at $\mathrm{rt}$ for $5 \mathrm{~h}$. The crude product was purified by silica-gel column chromatography (petrol/EtOAc, 95:5 $\mathrm{R}_{f} .0 .38$ ) to give title compound $\mathbf{3 6}$ (37 mg, 91\%) as a colourless oil, with spectroscopic data in accordance with the literature. ${ }^{26}{ }^{1} \mathrm{H}$ NMR $\left(300 \mathrm{MHz}, \mathrm{CDCl}_{3}\right) \delta_{\mathrm{H}}: 7.12-6.97(\mathrm{~m}, 2 \mathrm{H}, \operatorname{ArC}(2,6) H), 6.89-$ $6.75(\mathrm{~m}, 2 \mathrm{H}, \operatorname{ArC}(3,5) H), 5.74-5.53\left(\mathrm{~m}, 2 \mathrm{H}, \mathrm{CH}_{2} \mathrm{CHCH}_{2}\right), 5.11-4.80\left(\mathrm{~m}, 4 \mathrm{H}, \mathrm{CH}_{2} \mathrm{CHCH}_{2}\right)$, $3.78\left(\mathrm{~s}, 3 \mathrm{H}, \mathrm{OCH}_{3}\right), 2.63-2.58\left(\mathrm{~m}, 1 \mathrm{H}, \mathrm{CH}_{2} \mathrm{CH}\right), 2.45-2.20\left(\mathrm{~m}, 4 \mathrm{H}, \mathrm{CH}_{2} \mathrm{CH}\right.$ and $\left.\mathrm{CH}_{2} \mathrm{CH}\right)$.

\section{1-Methoxy-4-(2-methylpent-4-en-2-yl)benzene, 37}<smiles>C=CCC(C)(C)c1ccc(OC)cc1</smiles>

Following General Procedure G, 2-(4-methoxyphenyl)propan-2-ol S31 (33 mg, 0.2 mmol), allyltrimethylsilane 26 (64 $\mu \mathrm{L}, 0.4 \mathrm{mmol})$, pentafluorophenylboronic acid $\mathbf{1}(2 \mathrm{mg}, 10 \mu \mathrm{mol})$ and oxalic acid $2(1 \mathrm{mg}, 20 \mu \mathrm{mol})$ in $\mathrm{MeNO}_{2}(4.0 \mathrm{~mL})$ were reacted at $\mathrm{rt}$ for $16 \mathrm{~h}$. The crude 
product was purified by silica-gel column chromatography (petrol/EtOAc, 95:5 $\mathrm{R}_{f}: 0.29$ ) to give title compound $\mathbf{3 7}$ (26 mg, 68\%) as a colourless oil, with spectroscopic data in accordance with the literature. ${ }^{27}{ }^{1} \mathrm{H}$ NMR $\left(300 \mathrm{MHz}, \mathrm{CDCl}_{3}\right) \delta_{\mathrm{H}}$ : 7.27-7.25 (m, 2H, $\left.\operatorname{ArC}(2,6) H\right), 6.86-$ $6.83(\mathrm{~m}, 2 \mathrm{H}, \operatorname{ArC}(3,5) H), 5.64-5.48\left(\mathrm{~m}, 1 \mathrm{H}, \mathrm{CH}_{2} \mathrm{CHCH}_{2}\right), 4.99-4.93\left(\mathrm{~m}, 2 \mathrm{H}, \mathrm{CH}_{2} \mathrm{CHCH}_{2}\right)$, $3.80\left(\mathrm{~s}, 3 \mathrm{H}, \mathrm{OCH}_{3}\right), 2.33\left(\mathrm{t}, J=7.3 \mathrm{~Hz}, 2 \mathrm{H}, \mathrm{CH}_{2} \mathrm{CH}\right), 1.28\left(\mathrm{~s}, 6 \mathrm{H}, \mathrm{C}\left(\mathrm{CH}_{3}\right)_{2}\right)$.

\section{1-Methoxy-4-(3-phenylpent-4-en-2-yl)benzene, 38}

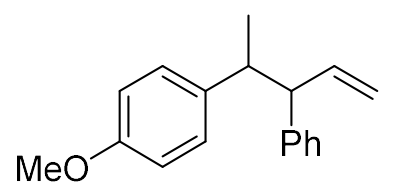

Cinnamyltrimethylsilane $\mathbf{S 3 3}$ (76 $\mathrm{mg}, 0.4 \mathrm{mmol}$ ) was added to a solution of pentafluorophenylboronic acid $1(4.2 \mathrm{mg}, 20 \mu \mathrm{mol})$ and oxalic acid $2(3.6 \mathrm{mg}, 40 \mu \mathrm{mol})$ in $\mathrm{MeNO}_{2}(8.0 \mathrm{~mL})$ were stirred at rt for 5 mins. 1-(4-Methoxyphenyl)ethan-1-ol S19 (30 mg, 0.2 mmol) was added and the reaction reacted at $\mathrm{rt}$ for $16 \mathrm{~h}$. The reaction was diluted with toluene and concentrated under reduced pressure to give the crude product, which was further purified by silica-gel column chromatography (Petrol/EtOAc, 99:1 to 95:5 $\mathrm{R}_{f}: 0.23$ ) to give $\mathbf{3 8}$ as a mixture of diastereoisomers (64:36 dr, $73 \mathrm{mg}, 77 \%)$ as a colourless oil. $v_{\max } 3093(\mathrm{C}-\mathrm{H}), 2968$ $(\mathrm{C}-\mathrm{H}), 1629(\mathrm{C}=\mathrm{C}), 1537(\mathrm{C}=\mathrm{C})$,

Data for major diastereoisomer: ${ }^{1} \mathrm{H}$ NMR $\left(300 \mathrm{MHz}, \mathrm{CDCl}_{3}\right)$ (selected) $\delta_{\mathrm{H}}: 7.38-7.27(\mathrm{~m}, 1 \mathrm{H}$, ArCH), 7.25-7.03 (m, 4H, ArCH), 7.03-6.96 (m, 2H, ArCH), 6.95-6.88 (m, 2H, ArCH), 6.08$5.99\left(\mathrm{~m}, 1 \mathrm{H}, \mathrm{CHCH}_{2}\right), 5.15-5.02\left(\mathrm{~m}, 2 \mathrm{H}, \mathrm{CHCH}_{2}\right), 3.72\left(\mathrm{~s}, 3 \mathrm{H}, \mathrm{OCH}_{3}\right), 3.41-3.30(\mathrm{~m}, 1 \mathrm{H}$, $\mathrm{PhCHCH}), 3.09-2.94\left(\mathrm{~m}, 1 \mathrm{H}, \mathrm{CHCH}_{3}\right), 1.30\left(\mathrm{~d}, J=7.0 \mathrm{~Hz}, 3 \mathrm{H}, \mathrm{CHCH}_{3}\right) ;{ }^{13} \mathrm{C}\left\{{ }^{1} \mathrm{H}\right\} \mathrm{NMR}(126$ $\left.\mathrm{MHz}, \mathrm{CDCl}_{3}\right) \delta_{\mathrm{C}}: 157.7(\mathrm{ArC}(4) \mathrm{OMe}), 143.6(\mathrm{ArC}(1)), 140.8\left(\mathrm{CHCH}_{2}\right), 137.5(\mathrm{ArCH}), 128.2$ $(\mathrm{ArCH} \times 2), 126.4(\mathrm{ArC}(2,6) \mathrm{H}), 125.9(\mathrm{ArCH}), 115.5\left(\mathrm{CHCH}_{2}\right), 113.4(\mathrm{ArC}(3,5) \mathrm{H}), 57.7$ $\left(\mathrm{OCH}_{3}\right), 55.2\left(\mathrm{CHCH}_{2} \mathrm{CH}\right), 44.2\left(\mathrm{CHCH}_{3}\right), 20.5\left(\mathrm{CHCH}_{3}\right) ; \mathrm{HRMS}\left(\mathrm{ESI}^{-}\right) \mathrm{m} / z$ : $\left[\mathrm{M}+\mathrm{CH}_{3} \mathrm{COO}^{-}\right.$ ] calcd for $\mathrm{C}_{20} \mathrm{H}_{23} \mathrm{O}_{3} 311.1653$, found 311.1656 .

Data for minor diastereoisomer: ${ }^{1} \mathrm{H}$ NMR $\left(300 \mathrm{MHz}, \mathrm{CDCl}_{3}\right)$ (selected) $\delta_{\mathrm{H}}: 7.38-7.27(\mathrm{~m}, 1 \mathrm{H}$, $\mathrm{ArCH}$ ), 7.25-7.03 (m, 4H, ArCH), 6.87-6.80 (m, 2H, ArCH), 6.72-6.64 (m, 2H, ArCH), 5.92$5.77\left(\mathrm{~m}, 1 \mathrm{H}, \mathrm{CHCH}_{2}\right), 4.90-4.68\left(\mathrm{~m}, 2 \mathrm{H}, \mathrm{CHCH}_{2}\right), 3.80\left(\mathrm{~s}, 3 \mathrm{H}, \mathrm{OCH}_{3}\right), 3.41-3.30(\mathrm{~m}, 1 \mathrm{H}$, $\mathrm{PhCHCH}), 3.09-2.94\left(\mathrm{~m}, 1 \mathrm{H}, \mathrm{CHCH}_{3}\right), 1.06\left(\mathrm{~d}, J=6.9 \mathrm{~Hz}, 3 \mathrm{H}, \mathrm{CHCH}_{3}\right) ;{ }^{13} \mathrm{C}\left\{{ }^{1} \mathrm{H}\right\} \mathrm{NMR}(126$ $\left.\mathrm{MHz}, \mathrm{CDCl}_{3}\right) \delta_{\mathrm{C}}: 158.0(\mathrm{ArC}(4) \mathrm{OMe}), 128.8(\mathrm{ArCH} \times 2), 126.8(\mathrm{ArC}(2,6) \mathrm{H}), 115.6\left(\mathrm{CHCH}_{2}\right)$, 113.6 $(\mathrm{ArC}(3,5) \mathrm{H}), 58.5\left(\mathrm{OCH}_{3}\right), 55.3\left(\mathrm{CHCH}_{2} \mathrm{CH}\right), 44.7\left(\mathrm{CHCH}_{3}\right), 21.0\left(\mathrm{CHCH}_{3}\right)$. 


\section{Control Reactions}

1,3-Diphenyl-2-[(2E)-3-phenylprop-2-en-1-yl]propane-1,3-dione, S13, and 1,3-Diphenyl2-(1-phenylallyl)propane-1,3-dione, S14<smiles>C=CC(c1ccccc1)C(C(=O)c1ccccc1)C(=O)c1ccccc1</smiles>

Following General Procedure C, cinnamyl alcohol S12 (54 mg, 0.4 mmol), 1,3diphenylpropane-1,3-dione (180 mg, $0.8 \mathrm{mmol}$ ), pentafluorophenylboronic acid $\mathbf{1}$ (4.2 mg, 20 $\mu \mathrm{mol})$ and oxalic acid $2(3.6 \mathrm{mg}, 40 \mu \mathrm{mol})$ in $\mathrm{MeNO}_{2}(8.0 \mathrm{~mL})$ were reacted at $90^{\circ} \mathrm{C}$ for $24 \mathrm{~h}$. The crude product was purified by silica-gel column chromatography (petrol/EtOAc, 95:5 to 85:15 $\left.\mathrm{R}_{f}: 0.21\right)$ to give an 87:13 mixture of S13:S14 (87 $\left.\mathrm{mg}, 64 \%\right)$ as a yellow solid, with spectroscopic data in accordance with the literature. ${ }^{16}$

S13 (major): ${ }^{1} \mathrm{H}$ NMR (400 MHz, $\left.\mathrm{CDCl}_{3}\right) \delta_{\mathrm{H}}: 7.99-7.95$ (m, 4H, $\left.\mathrm{ArCH}\right), 7.59-7.54(\mathrm{~m}, 2 \mathrm{H}$, $\operatorname{ArCH}), 7.47-7.43(\mathrm{~m}, 4 \mathrm{H}, \operatorname{ArCH}), 7.32-7.23(\mathrm{~m}, 5 \mathrm{H}, \operatorname{ArCH}), 6.46(\mathrm{~d}, J=15.9 \mathrm{~Hz}, 1 \mathrm{H}$, $\mathrm{PhCH}=\mathrm{CH}), 6.28-6.21(\mathrm{~m}, 1 \mathrm{H}, \mathrm{PhCH}=\mathrm{CH}), 5.35(\mathrm{t}, J=6.8 \mathrm{~Hz}, 1 \mathrm{H} \mathrm{C}(\mathrm{O}) \mathrm{CH}), 3.02(\mathrm{td}, J=$ 7.1, $\left.1.1 \mathrm{~Hz}, 2 \mathrm{H}, \mathrm{CH}_{2} \mathrm{CH}\right)$.

S14 (minor): ${ }^{1} \mathrm{H}$ NMR (400 MHz, $\left.\mathrm{CDCl}_{3}\right) \delta_{\mathrm{H}}:$ 8.04-8.02 (m, 2H, $\left.\operatorname{ArCH}\right), 7.79-7.75(\mathrm{~m}, 2 \mathrm{H}$, $\operatorname{ArCH}), 7.21-7.16$ (m, 8H, ArCH), 7.11-6.98 (m, 3H, $\mathrm{ArCH}), 6.06-5.97$ (m, $\left.1 \mathrm{H}, \mathrm{CH}=\mathrm{CH}_{2}\right)$, $5.88(\mathrm{~d}, J=10.6 \mathrm{~Hz}, 1 \mathrm{H}, \mathrm{C}(\mathrm{O}) \mathrm{CH}), 5.03-4.92\left(\mathrm{~m}, 2 \mathrm{H}, \mathrm{CH}=\mathrm{CH}_{2}\right), 4.66(\mathrm{dd}, J=10.6,8.0 \mathrm{~Hz}$, $1 \mathrm{H}, \mathrm{PhCCH})$.

The reaction was also performed using vinylbenzyl alcohol S11 (54 mg, $0.4 \mathrm{mmol})$, giving an $85: 15$ mixture of S13:S14 in 66\% yield, with spectroscopic data as above.

\section{(E)-Hexa-1,5-dien-1-ylbenzene, S15}

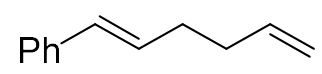

Following General Procedure G, cinnamyl alcohol S12 (54 mg, 0.4 mmol), allyltrimethylsilane 26 (130 $\mu 1,0.8 \mathrm{mmol})$, pentafluorophenylboronic acid $\mathbf{1}$ (4.2 $\mathrm{mg}, 20 \mu \mathrm{mol})$ and oxalic acid $2(3.6 \mathrm{mg}, 40 \mu \mathrm{mol})$ in $\mathrm{MeNO}_{2}(8.0 \mathrm{~mL})$ were reacted at $\mathrm{rt}$ for $16 \mathrm{~h} .{ }^{1} \mathrm{H} \mathrm{NMR}$ catalysis of the crude reaction mixture showed $100 \%$ conversion. The crude product was purified by silica-gel column chromatography (petrol/EtOAc, 100:0 to 90:10 $\mathrm{R}_{f}$ : 0.36 ) to give title compound S15 (57 mg, 66\%) as a colourless oil, with spectroscopic data in accordance with the literature. ${ }^{28}{ }^{1} \mathrm{H}$ NMR $\left(300 \mathrm{MHz}, \mathrm{CDCl}_{3}\right) \delta_{\mathrm{H}}: 7.38-7.28(\mathrm{~m}, 4 \mathrm{H}, \mathrm{ArCH}), 7.24-7.12(\mathrm{~m}$, $1 \mathrm{H}, \operatorname{ArC}(4) H), 6.47-6.35$ (m, 1H, $\mathrm{ArCC} H), 6.31-6.15$ (m, 1H, $\left.\mathrm{CHCHCH}_{2}\right), 5.96-5.78$ (m, 1H, 
$\left.\mathrm{CH}_{2} \mathrm{CHCH}_{2}\right), 5.13-4.95\left(\mathrm{~m}, 2 \mathrm{H}, \mathrm{CH}_{2} \mathrm{CHCH}_{2}\right), 2.38-2.18\left(\mathrm{~m}, 4 \mathrm{H}, \mathrm{CH}_{2} \mathrm{CH}_{2} \mathrm{CH}\right)$.

The reaction was also performed using vinylbenzyl alcohol S11 (54 mg, $0.4 \mathrm{mmol}$ ), giving S15 in $63 \%$ yield, with spectroscopic data as above. 
NMR Spectra 


\section{$3{ }^{1} \mathrm{H}$ NMR (500 MHz, $\left.\mathrm{CDCl}_{3}\right)$}
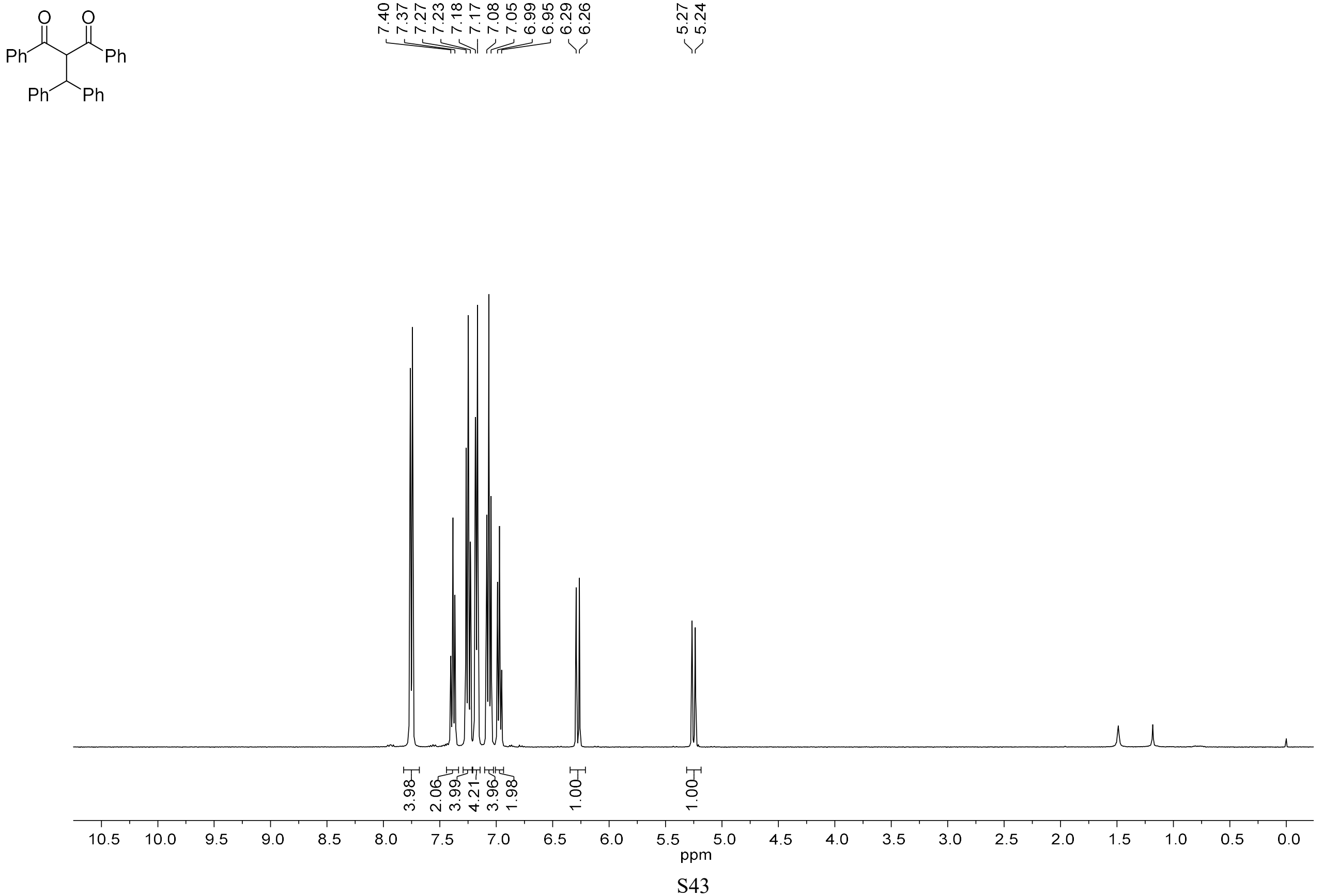
$4{ }^{1} \mathrm{H}$ NMR (500 MHz, $\mathrm{CDCl}_{3}$ )
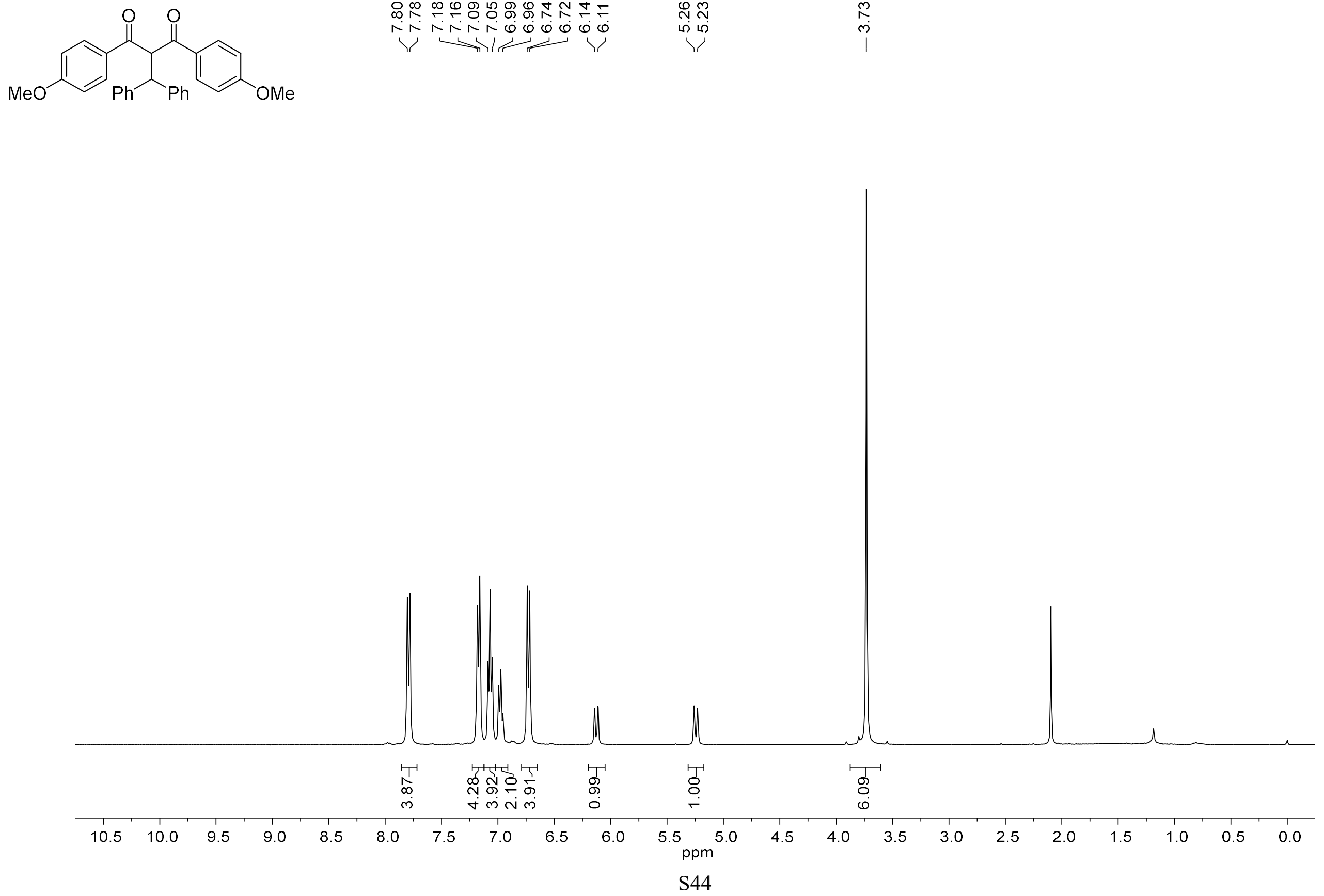
$5{ }^{1} \mathrm{H}$ NMR $\left(\mathrm{CDCl}_{3}, 500 \mathrm{MHz}\right)$

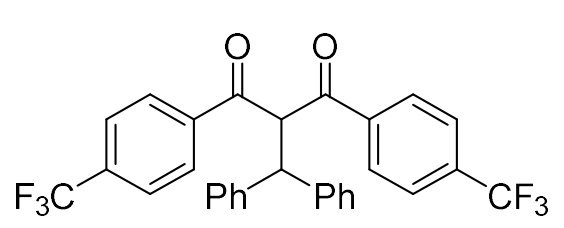

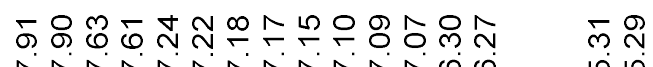

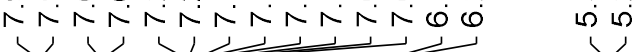

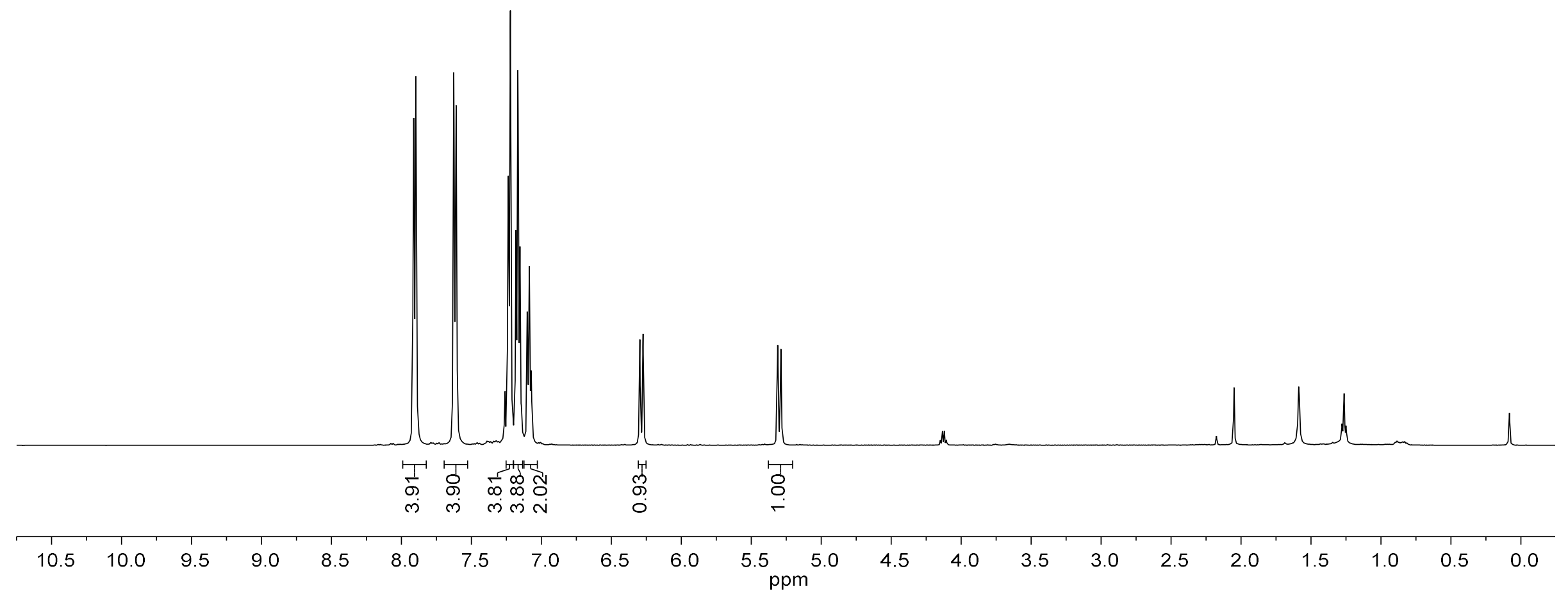

S45 
$5{ }^{13} \mathrm{C}\left\{{ }^{1} \mathrm{H}\right\} \mathrm{NMR}\left(126 \mathrm{MHz}, \mathrm{CDCl}_{3}\right)$

$$
\text { ڤั }
$$

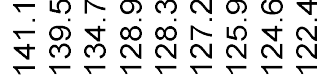

华资证

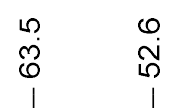
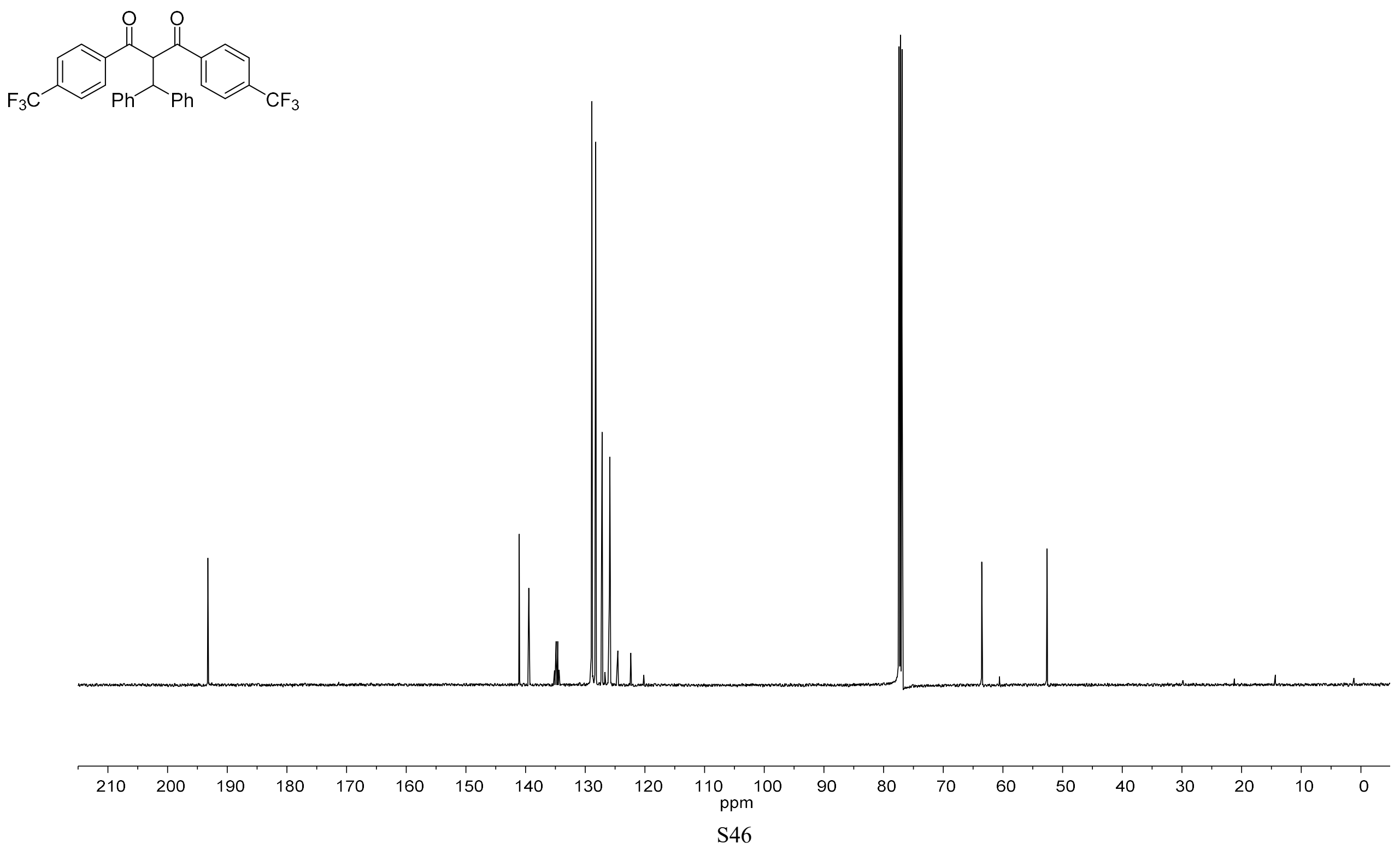
$5{ }^{19} \mathrm{~F}\left\{{ }^{1} \mathrm{H}\right\}$ NMR (471 MHz, $\left.\mathrm{CDCl}_{3}\right)$
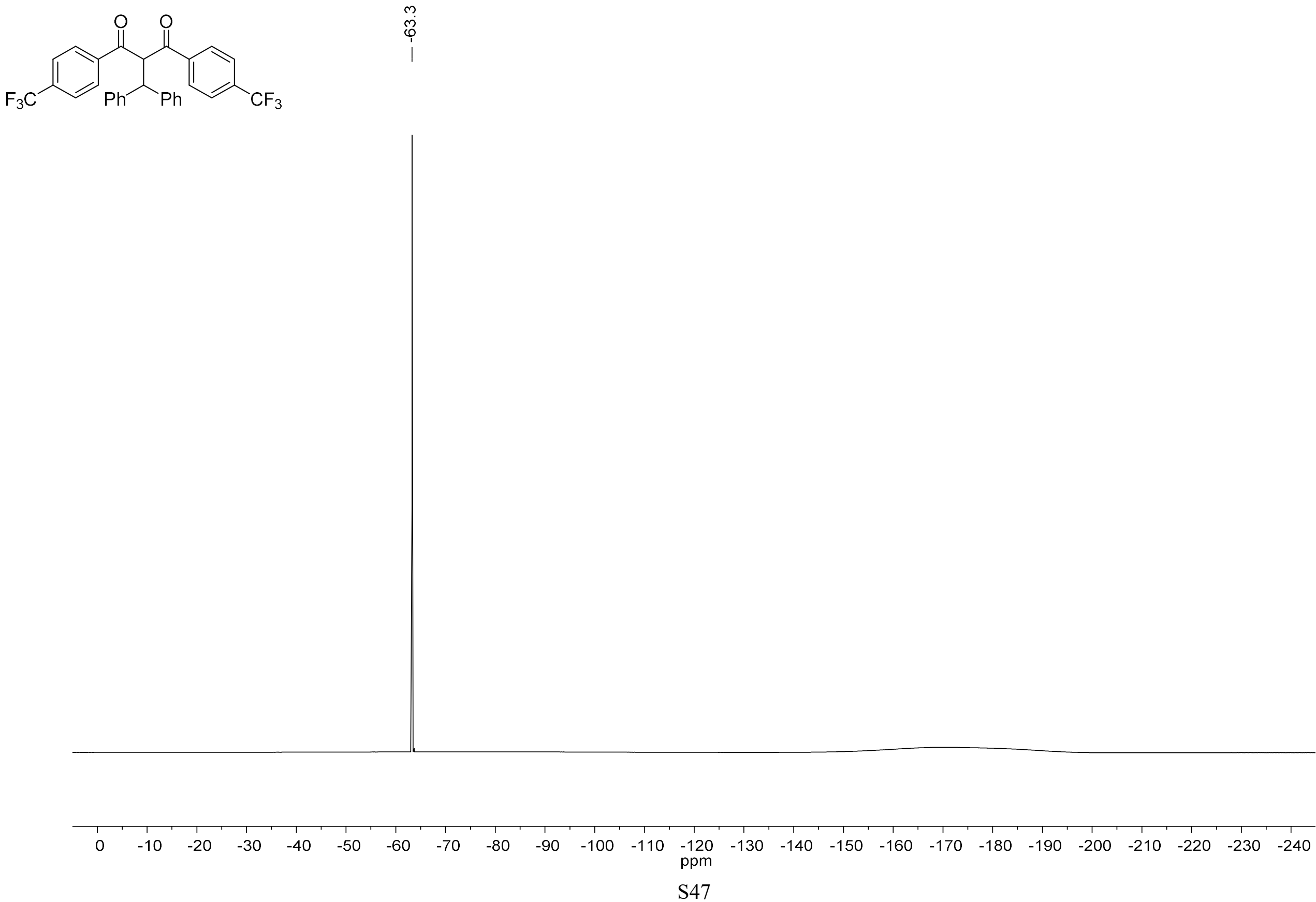
$6{ }^{1} \mathrm{H} \mathrm{NMR}\left(\mathrm{CDCl}_{3}, 500 \mathrm{MHz}\right)$

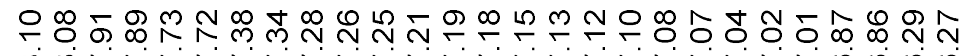

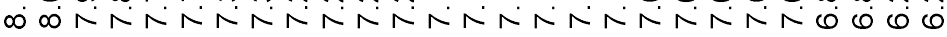
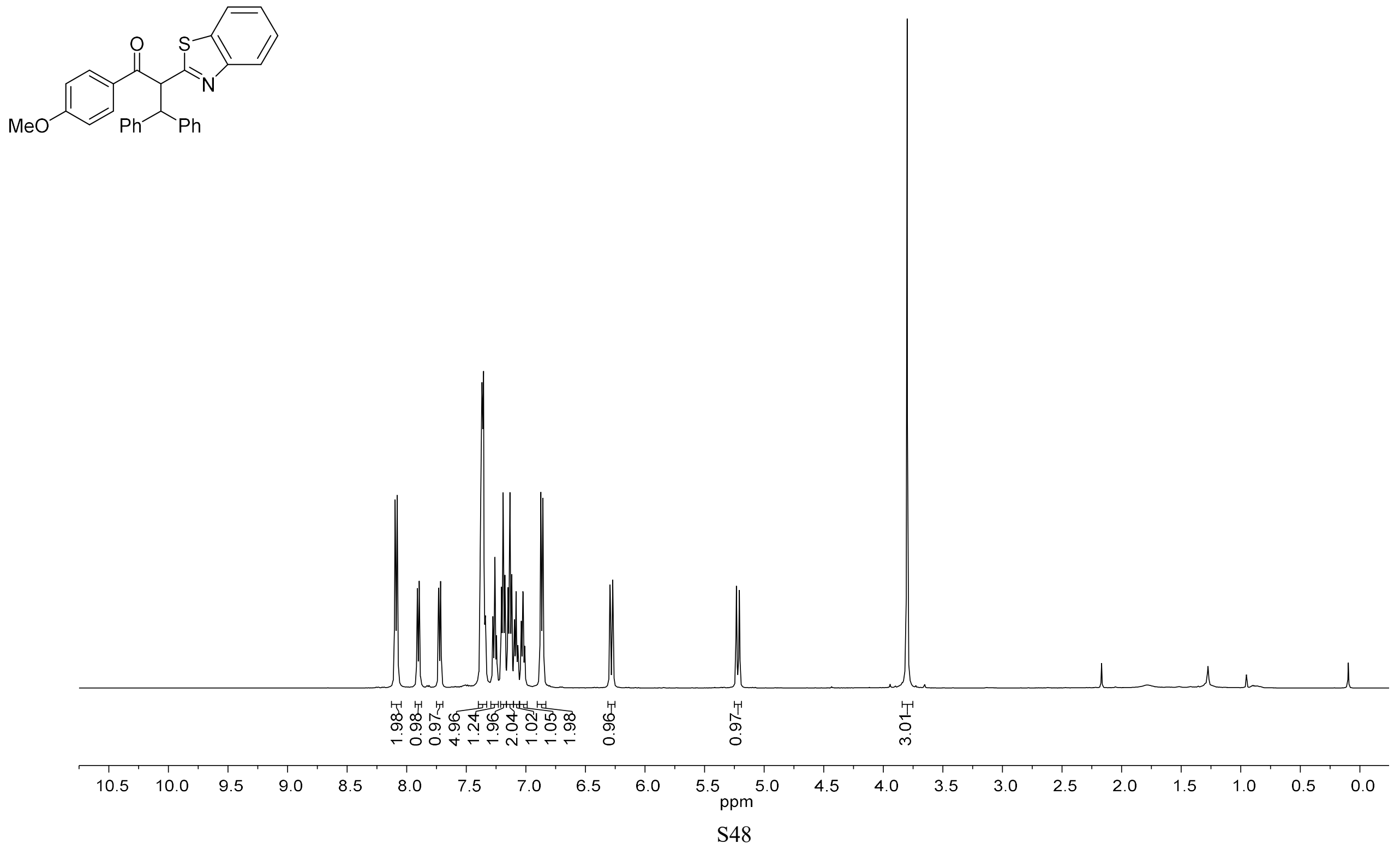
6 ${ }^{13} \mathrm{C}\left\{{ }^{1} \mathrm{H}\right\}$ NMR $\left(126 \mathrm{MHz}, \mathrm{CDCl}_{3}\right)$

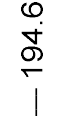
$\stackrel{1}{d}$

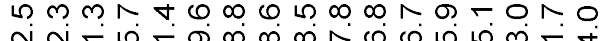

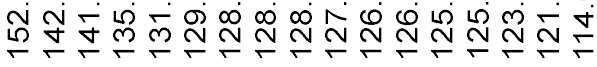
$\infty 00$
की
$i$
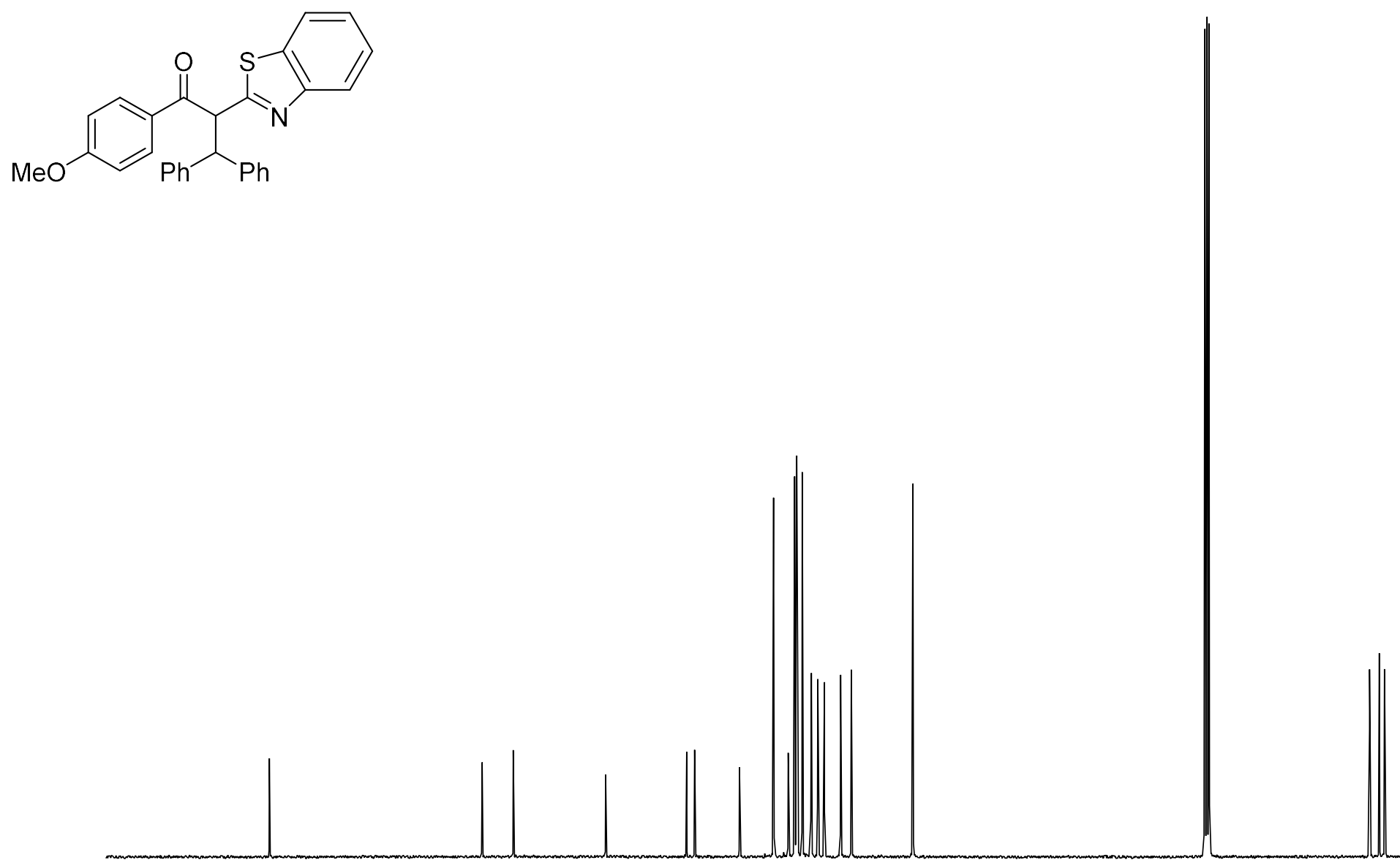
$7{ }^{1} \mathrm{H}$ NMR $\left(400 \mathrm{MHz}, \mathrm{CDCl}_{3}\right)$

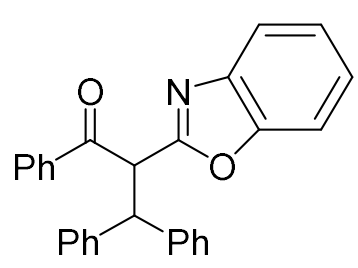

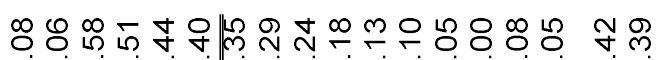

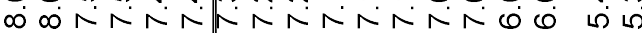

$\overbrace{\mathrm{Ph}}^{\mathrm{O}}$

$\checkmark$,

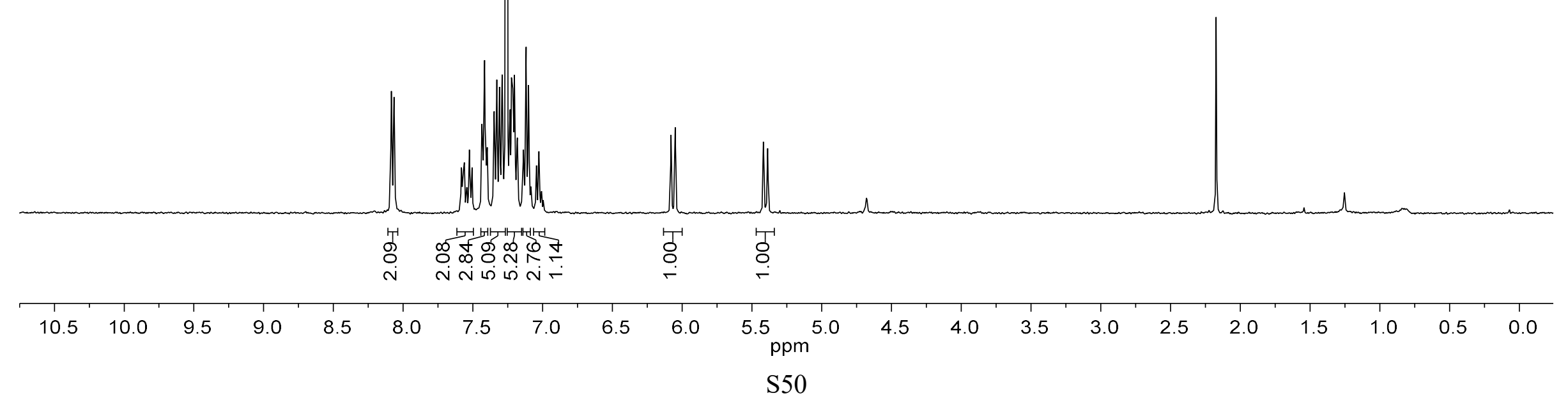


$7{ }^{13} \mathrm{C}\left\{{ }^{1} \mathrm{H}\right\} \mathrm{NMR}\left(126 \mathrm{MHz}, \mathrm{CDCl}_{3}\right)$

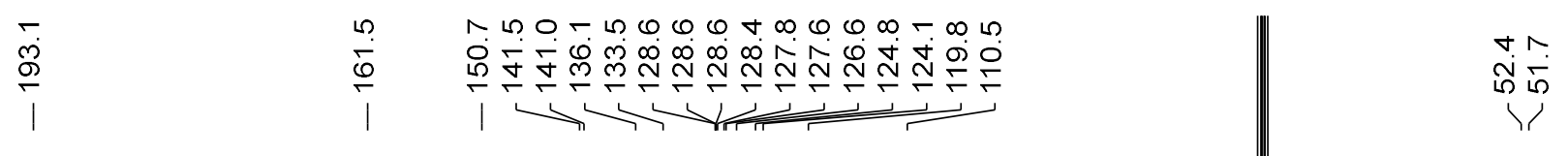
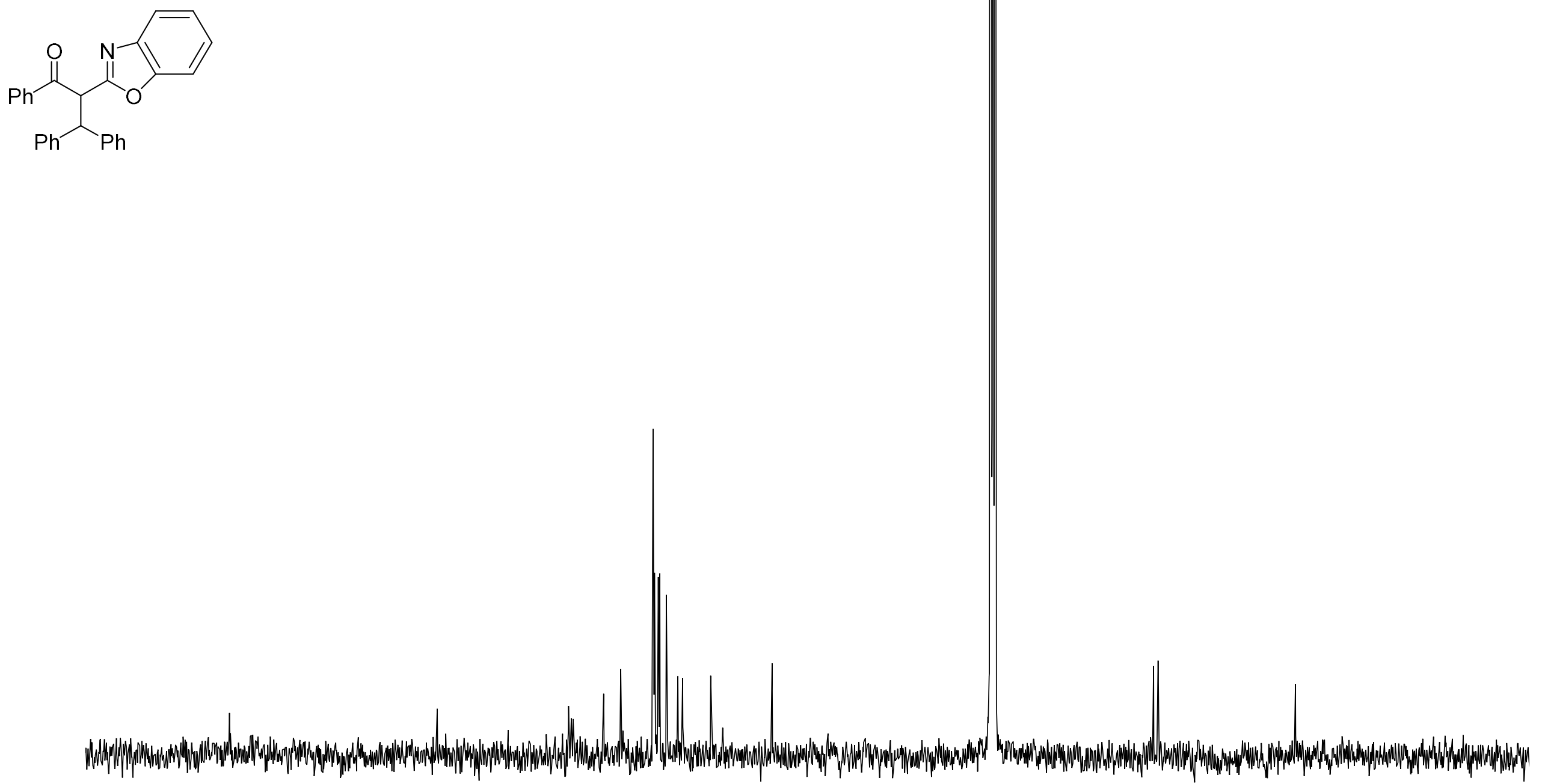

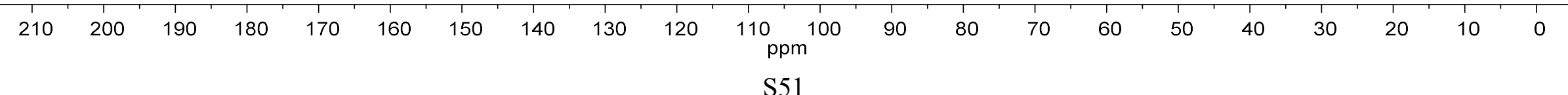


$8{ }^{1} \mathrm{H}$ NMR (300 MHz, $\left.\mathrm{CDCl}_{3}\right)$
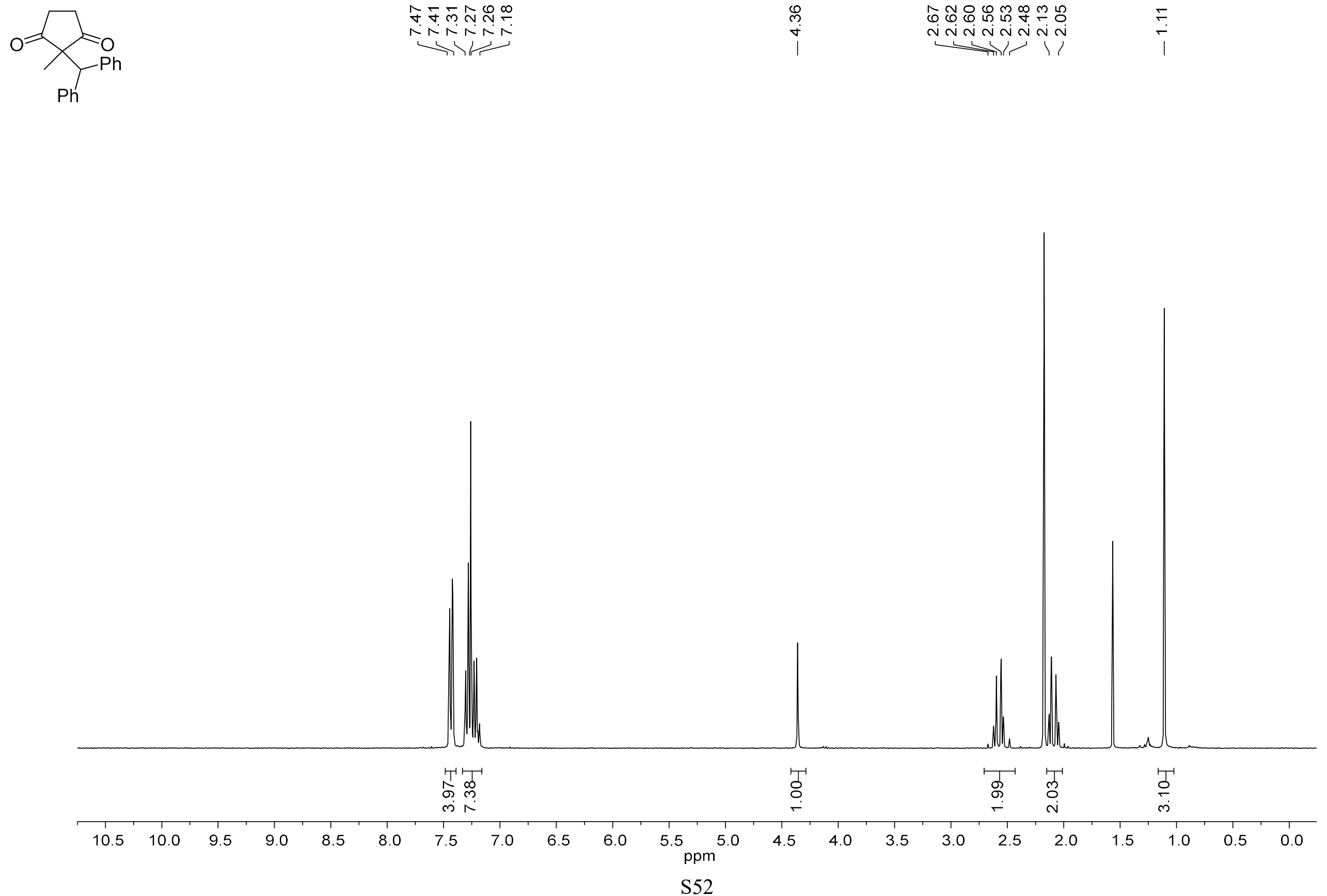
$8^{13} \mathrm{C}\left\{{ }^{1} \mathrm{H}\right\} \mathrm{NMR}\left(126 \mathrm{MHz}, \mathrm{CDCl}_{3}\right)$
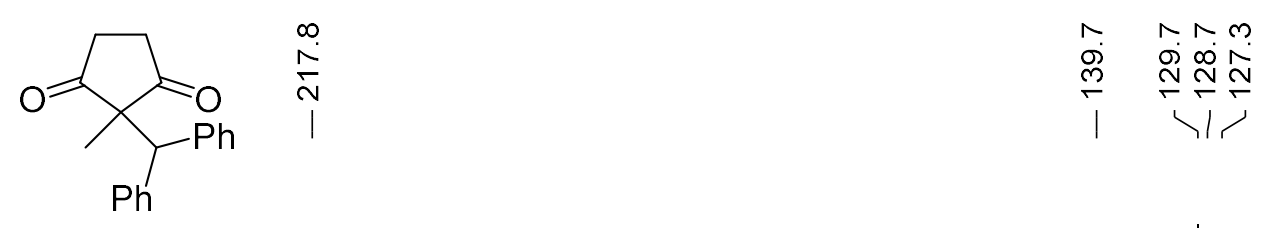

$\begin{array}{ll}10 & 0 \\ 0 & 0 \\ 0 & 0 \\ 0 & 1\end{array}$

$\begin{array}{cc}-\dot{0} & \stackrel{N}{\sim} \\ i & 1\end{array}$

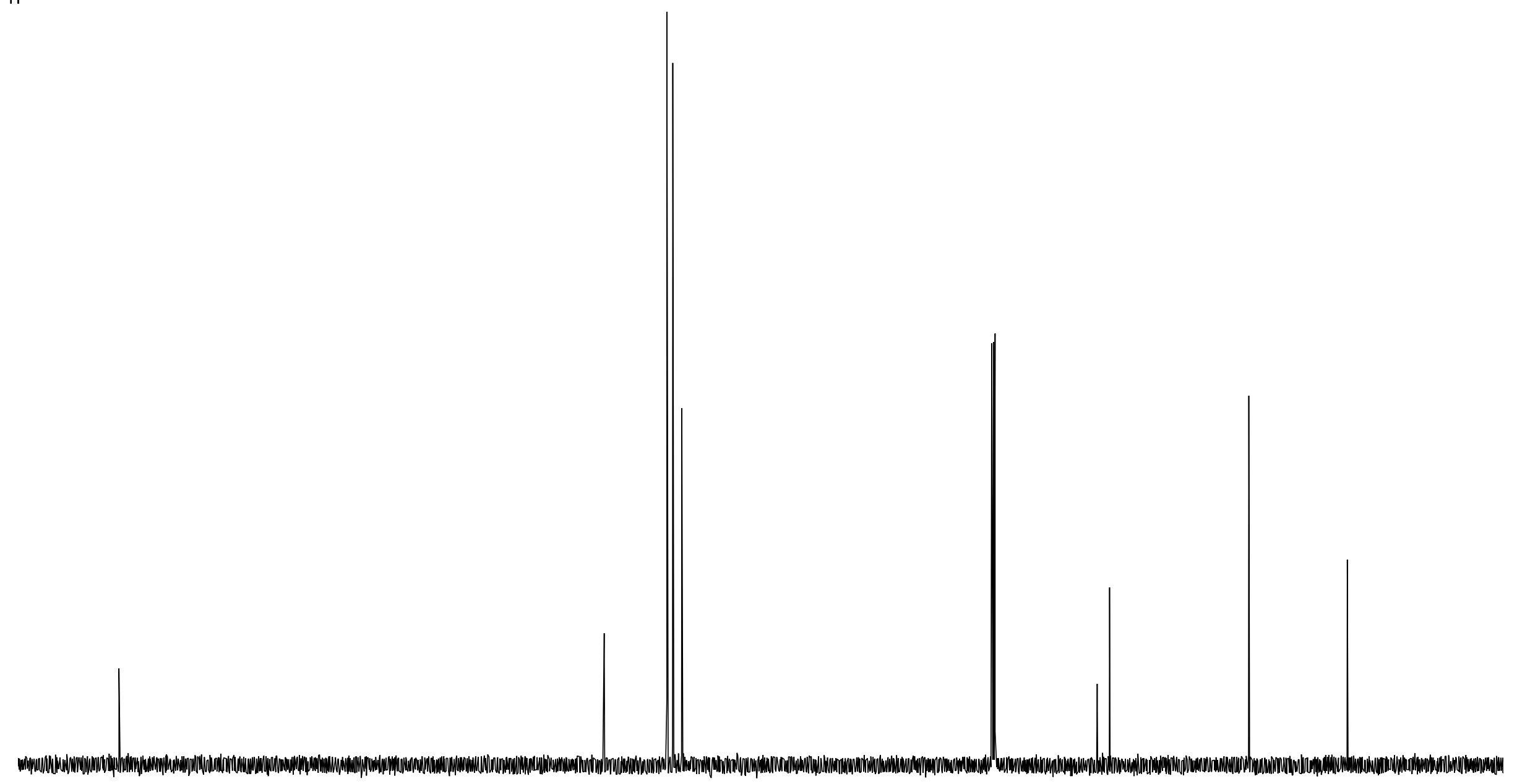

$\begin{array}{lllll}210 & 200 & 190 & 180 & 170\end{array}$

160150

140

130

120 


\section{$9{ }^{1} \mathrm{H}$ NMR (400 MHz, $\left.\mathrm{CDCl}_{3}\right)$}
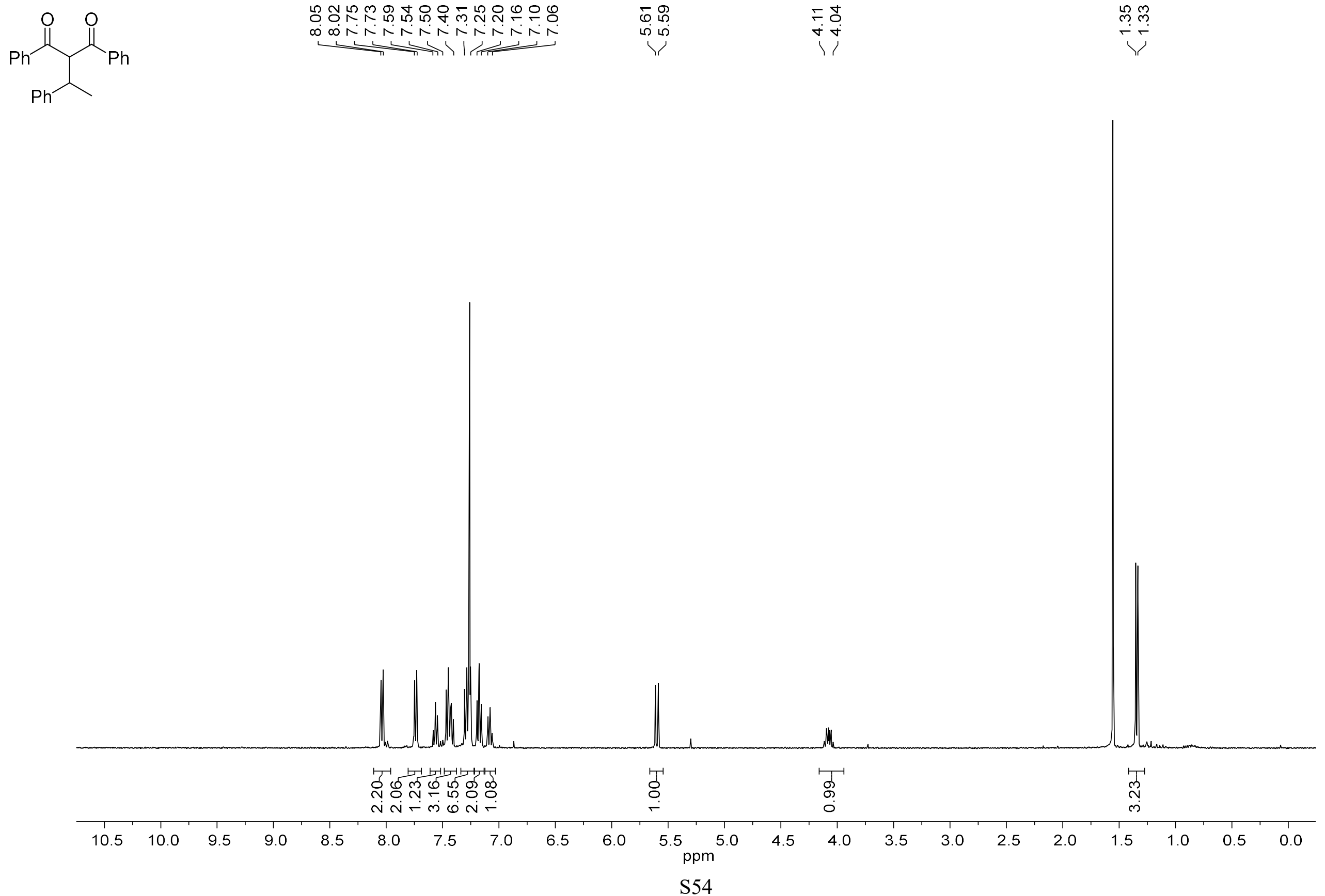
$10{ }^{1} \mathrm{H}$ NMR (400 MHz, $\mathrm{CDCl}_{3}$ )
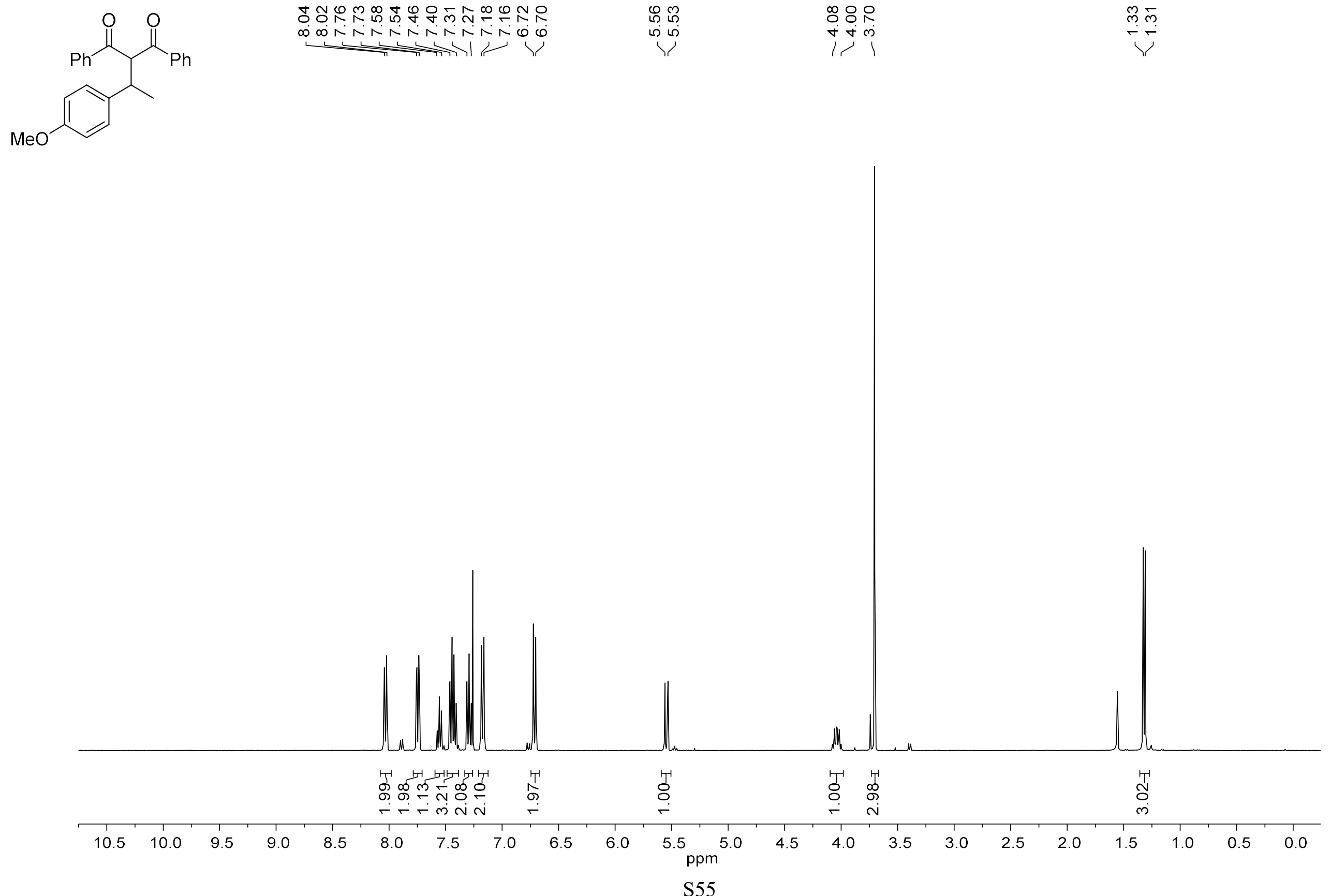
$11{ }^{1} \mathrm{H}$ NMR (400 MHz, $\mathrm{CDCl}_{3}$ )
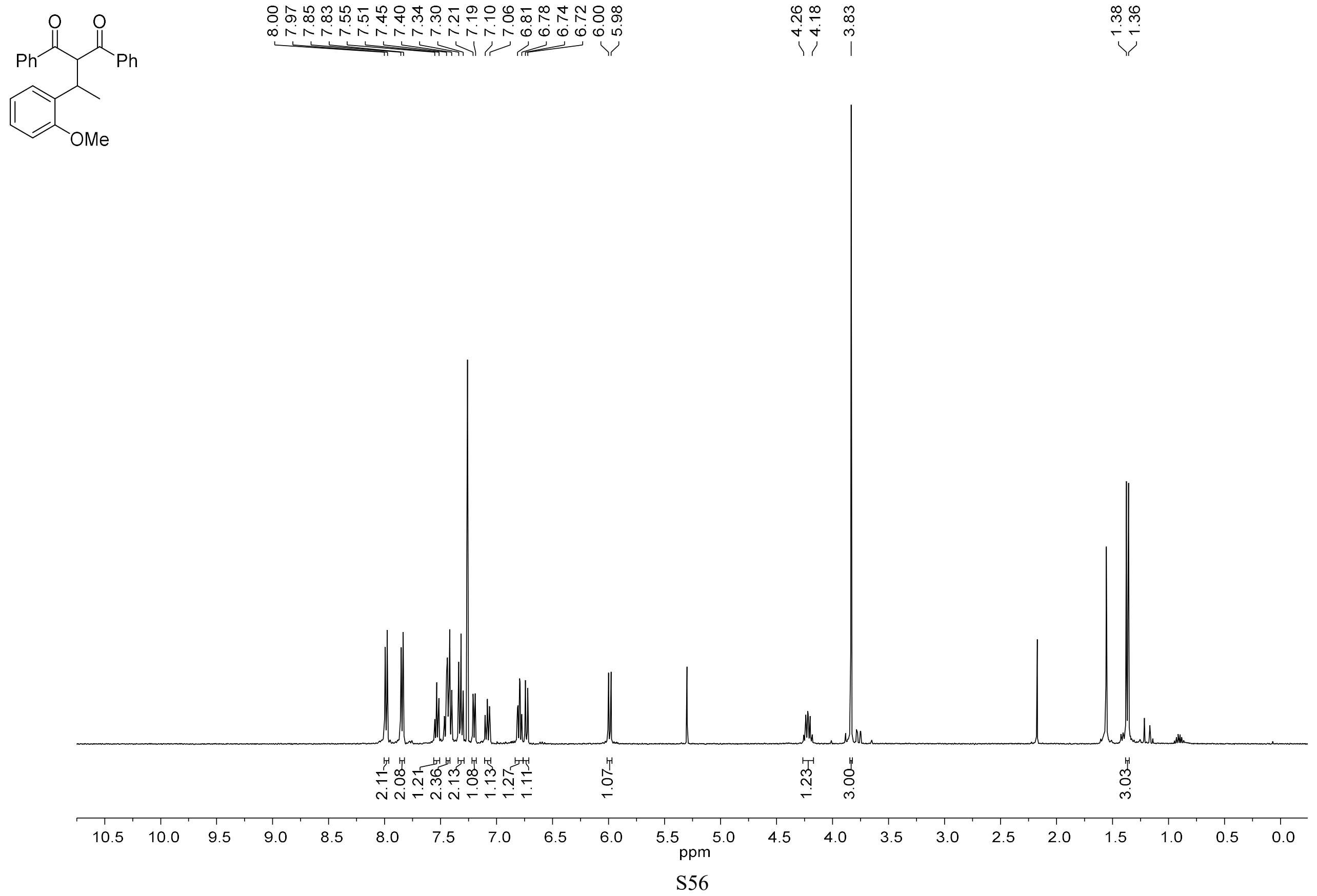
$11{ }^{13} \mathrm{C}\left\{{ }^{1} \mathrm{H}\right\}$ NMR $\left(126 \mathrm{MHz}, \mathrm{CDCl}_{3}\right)$

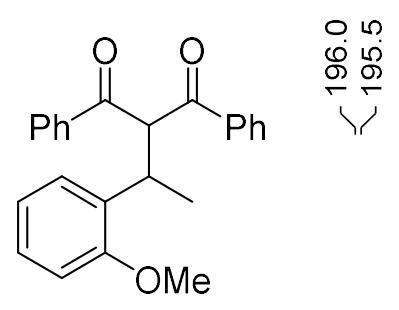


$12{ }^{1} \mathrm{H}$ NMR (400 MHz, $\mathrm{CDCl}_{3}$ )
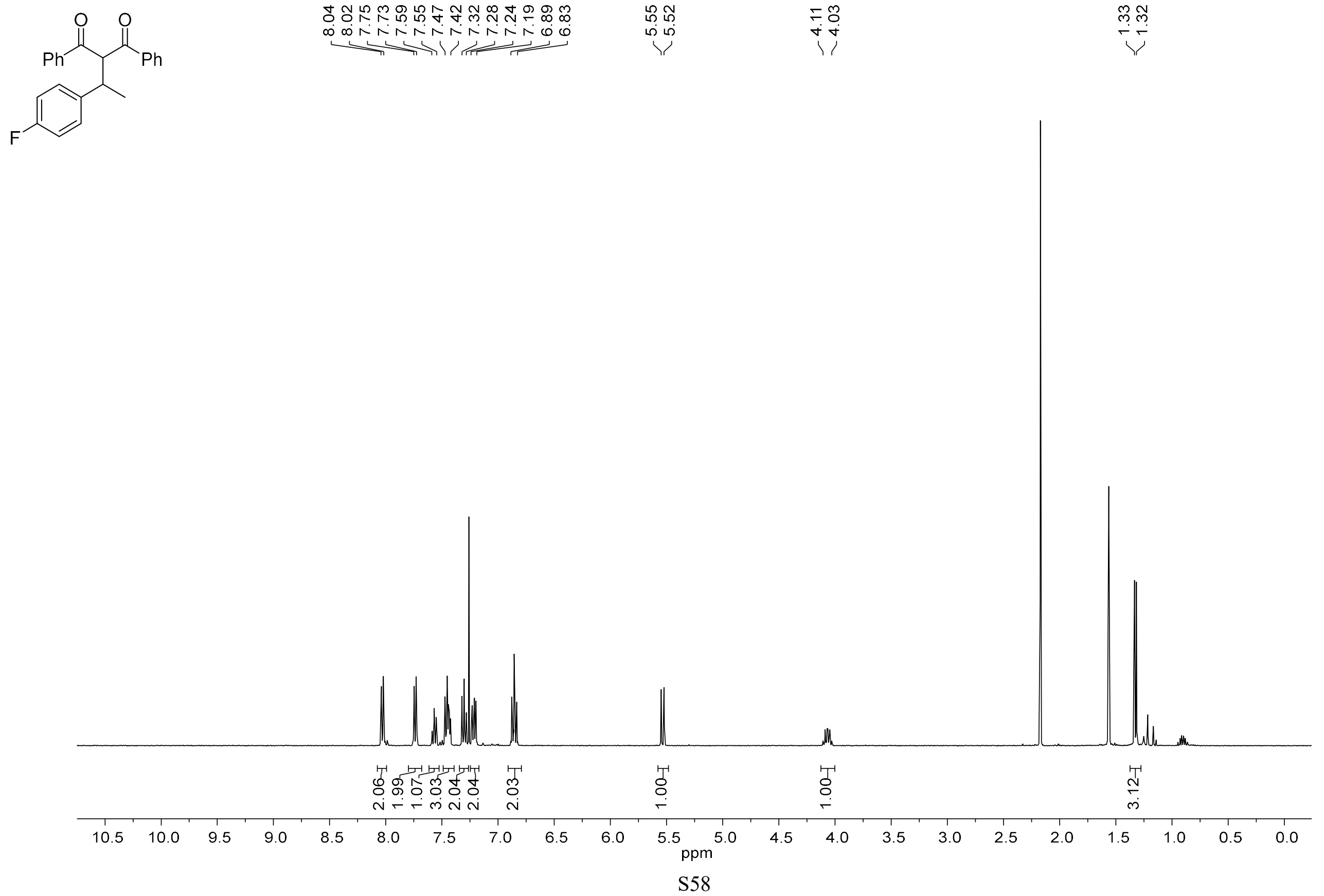
$13{ }^{1} \mathrm{H}$ NMR (400 MHz, $\mathrm{CDCl}_{3}$ )
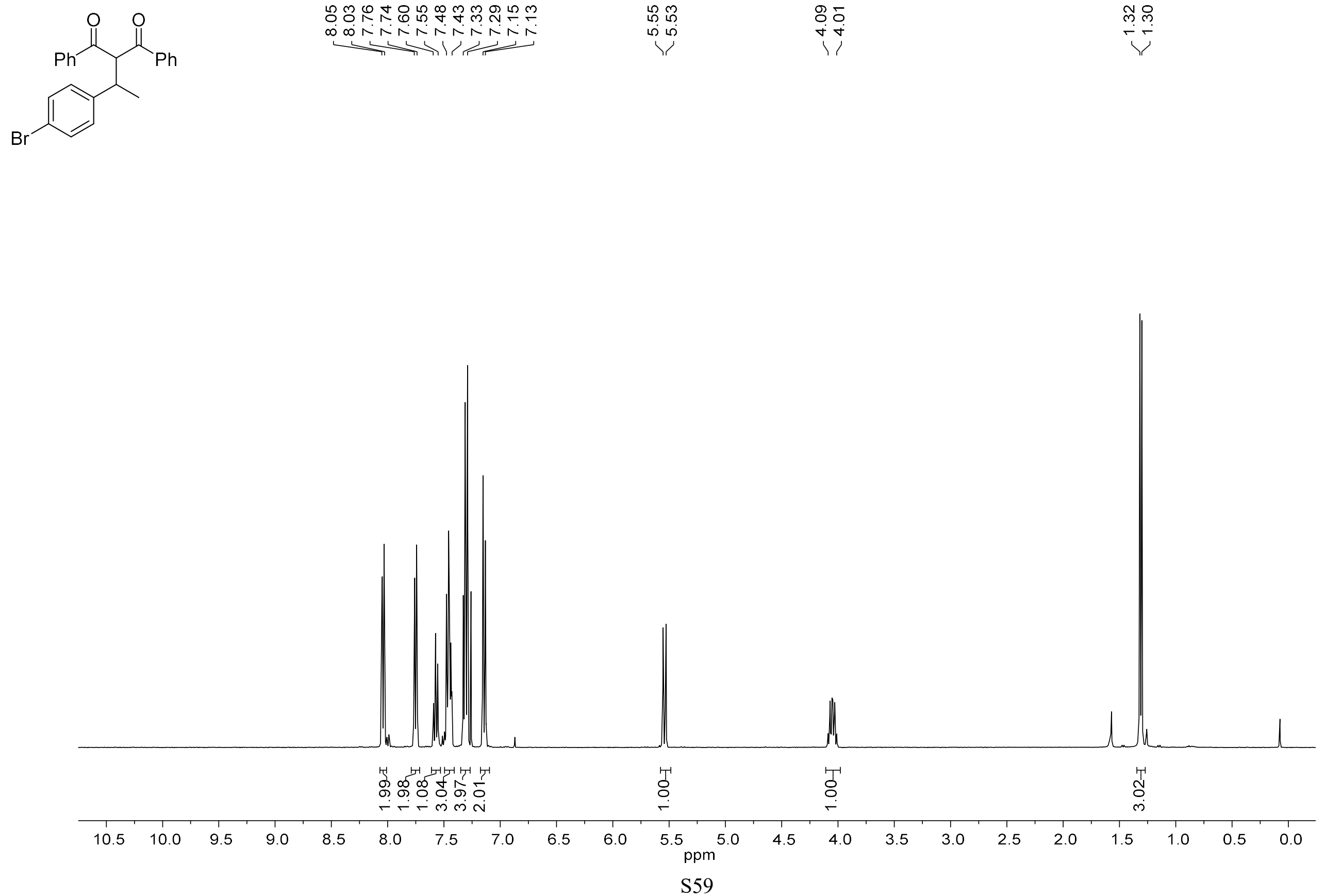
$14{ }^{1} \mathrm{H}$ NMR (300 MHz, $\left.\mathrm{CDCl}_{3}\right)$
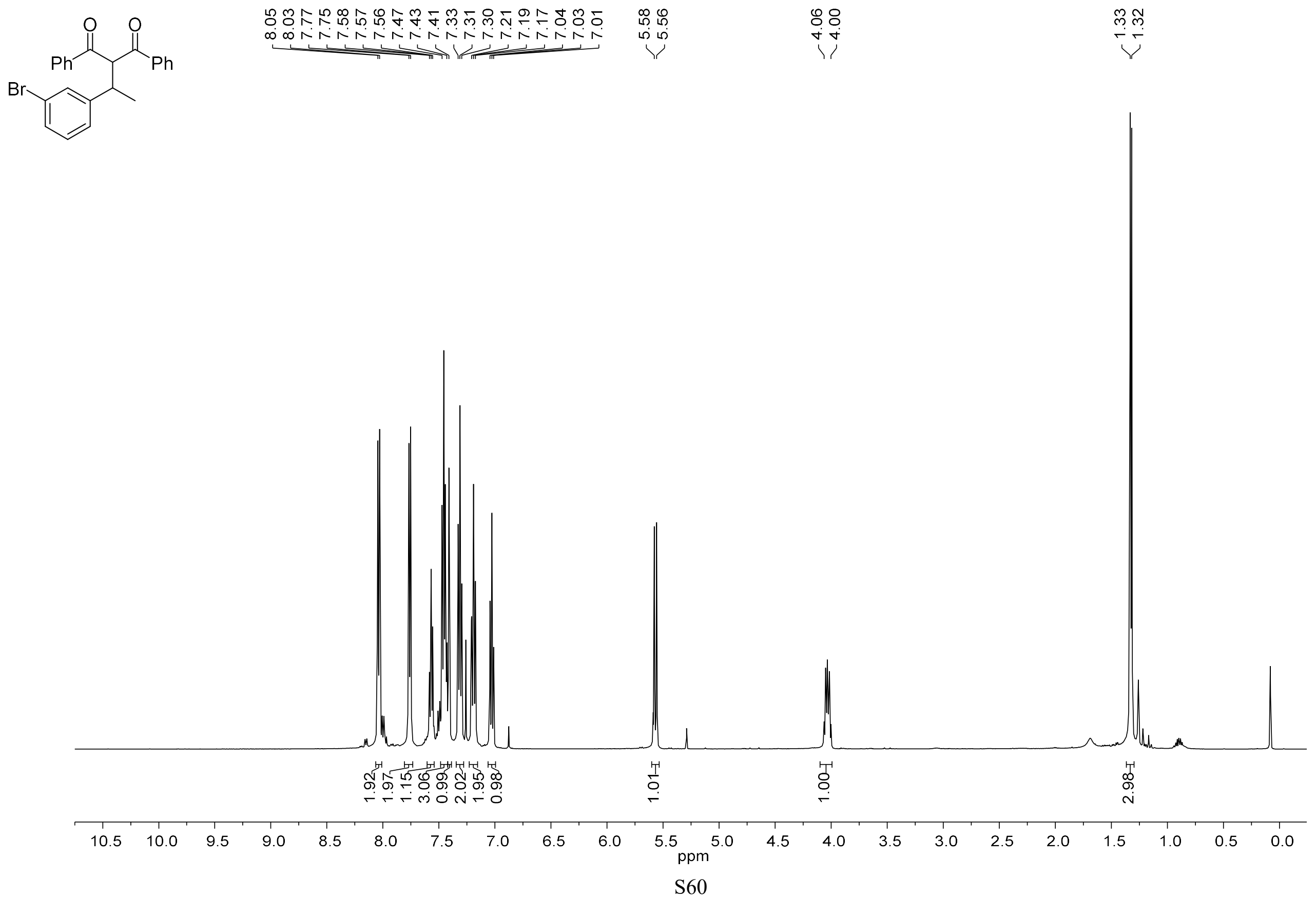
$14{ }^{13} \mathrm{C}\left\{{ }^{1} \mathrm{H}\right\}$ NMR $\left(126 \mathrm{MHz}, \mathrm{CDCl}_{3}\right)$

㝑芦

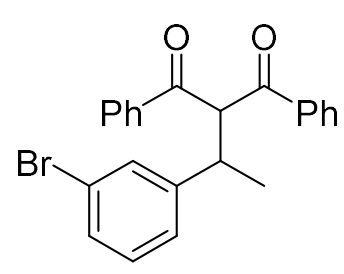

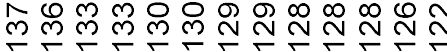
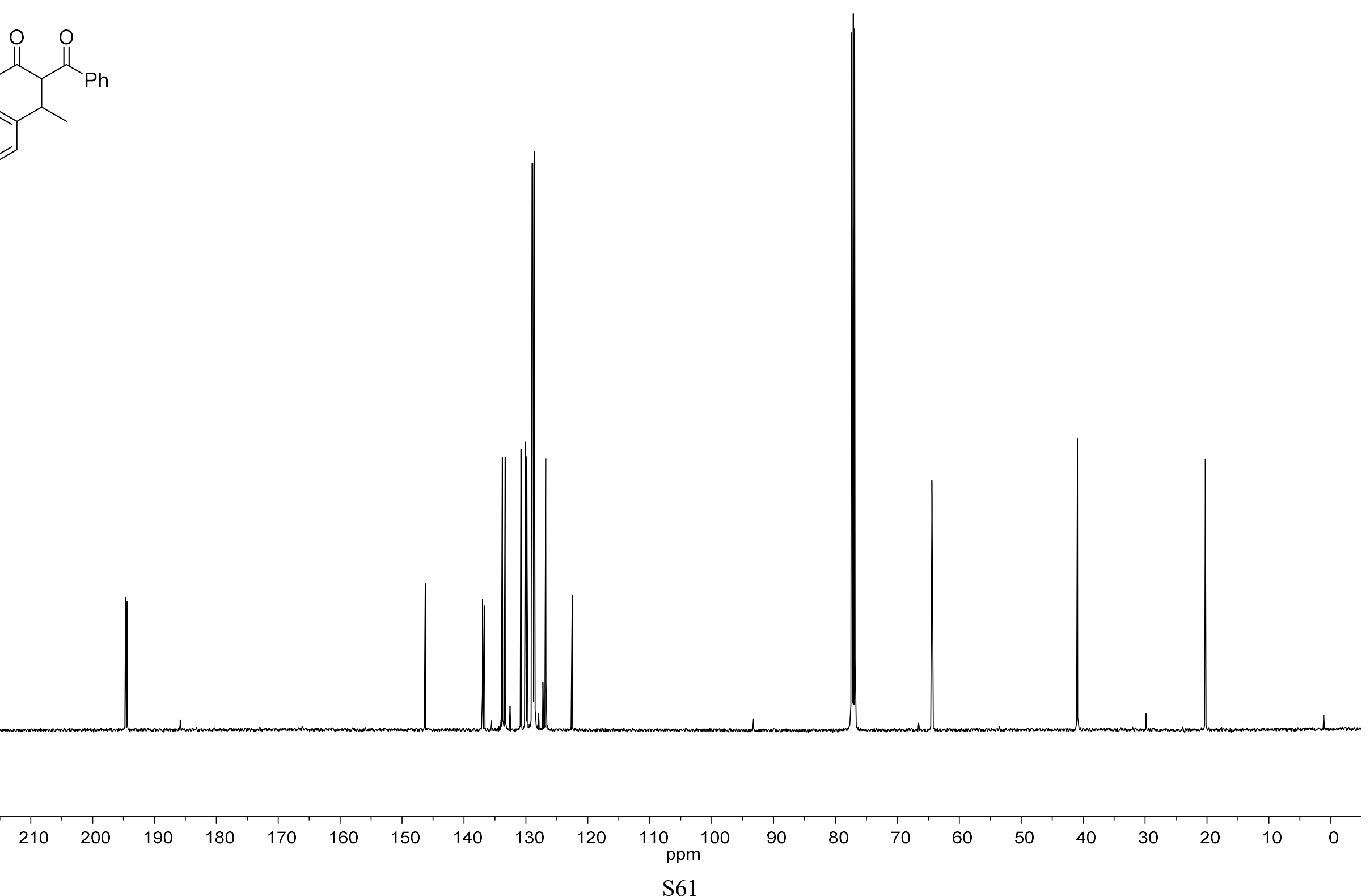
$15{ }^{1} \mathrm{H}$ NMR (300 MHz, $\mathrm{CDCl}_{3}$ )

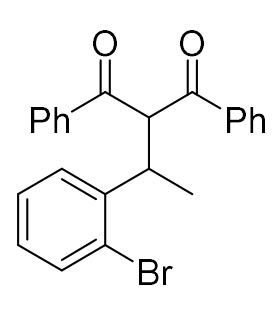

$\mathrm{Br}$

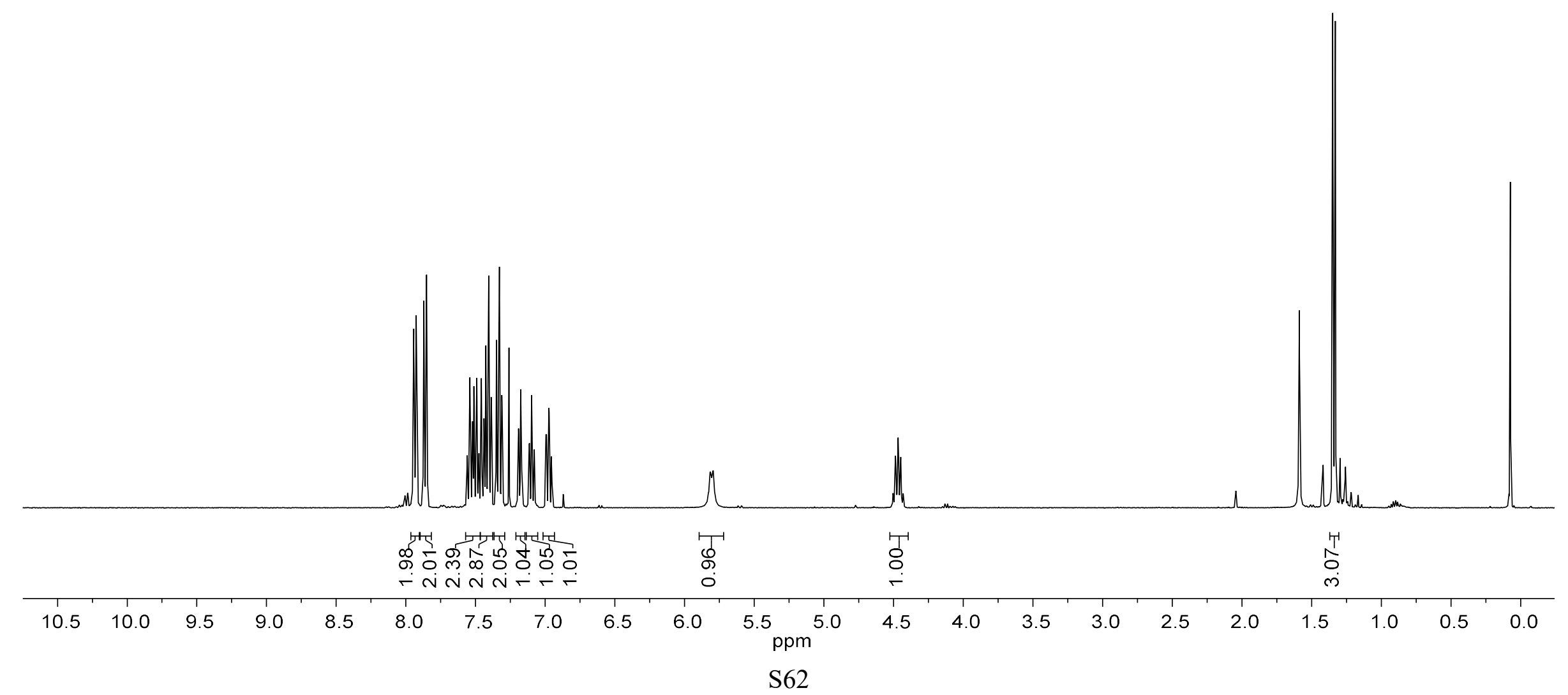


$16{ }^{1} \mathrm{H}$ NMR (300 MHz, $\mathrm{CDCl}_{3}$ )
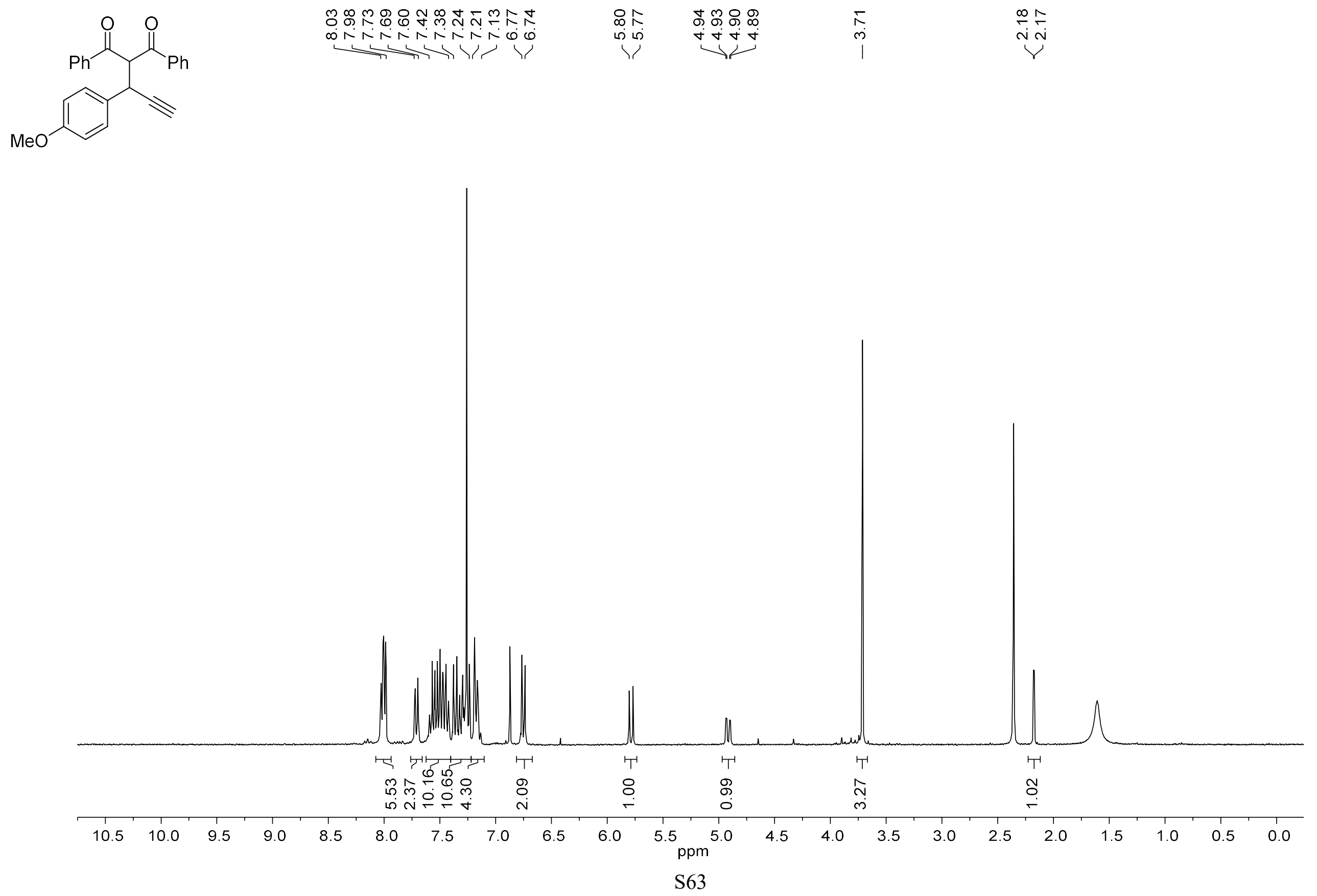
$16{ }^{13} \mathrm{C}\left\{{ }^{1} \mathrm{H}\right\}$ NMR $\left(126 \mathrm{MHz}, \mathrm{CDCl}_{3}\right)$

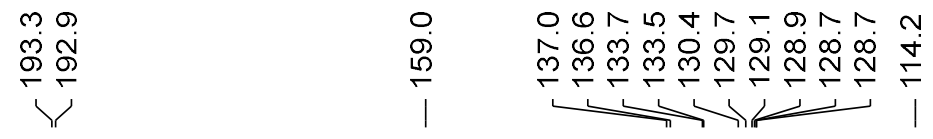

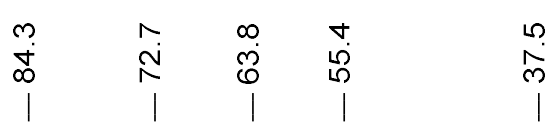

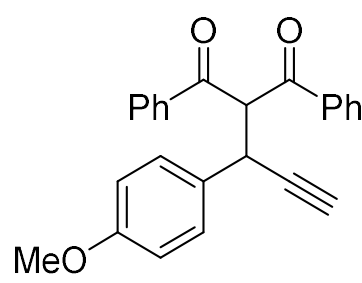

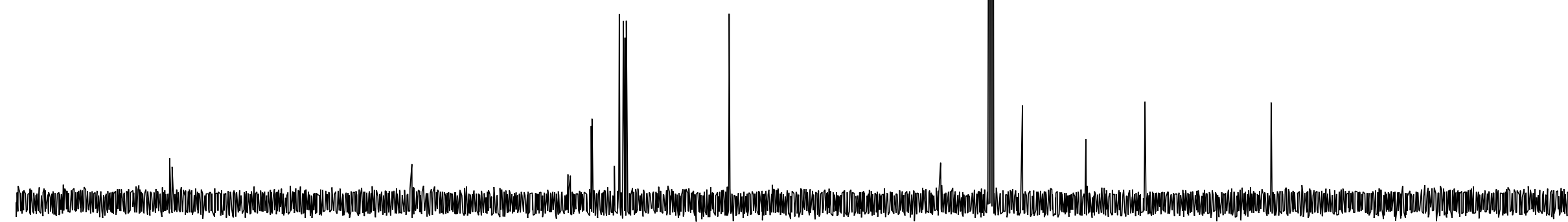

$160 \quad 150$

130

120

110100

$90 \quad 80$

70

60

$50 \quad 40$

$30 \quad 20$

10 
S17 ${ }^{1} \mathrm{H}$ NMR (300 MHz, $\mathrm{CDCl}_{3}$ )
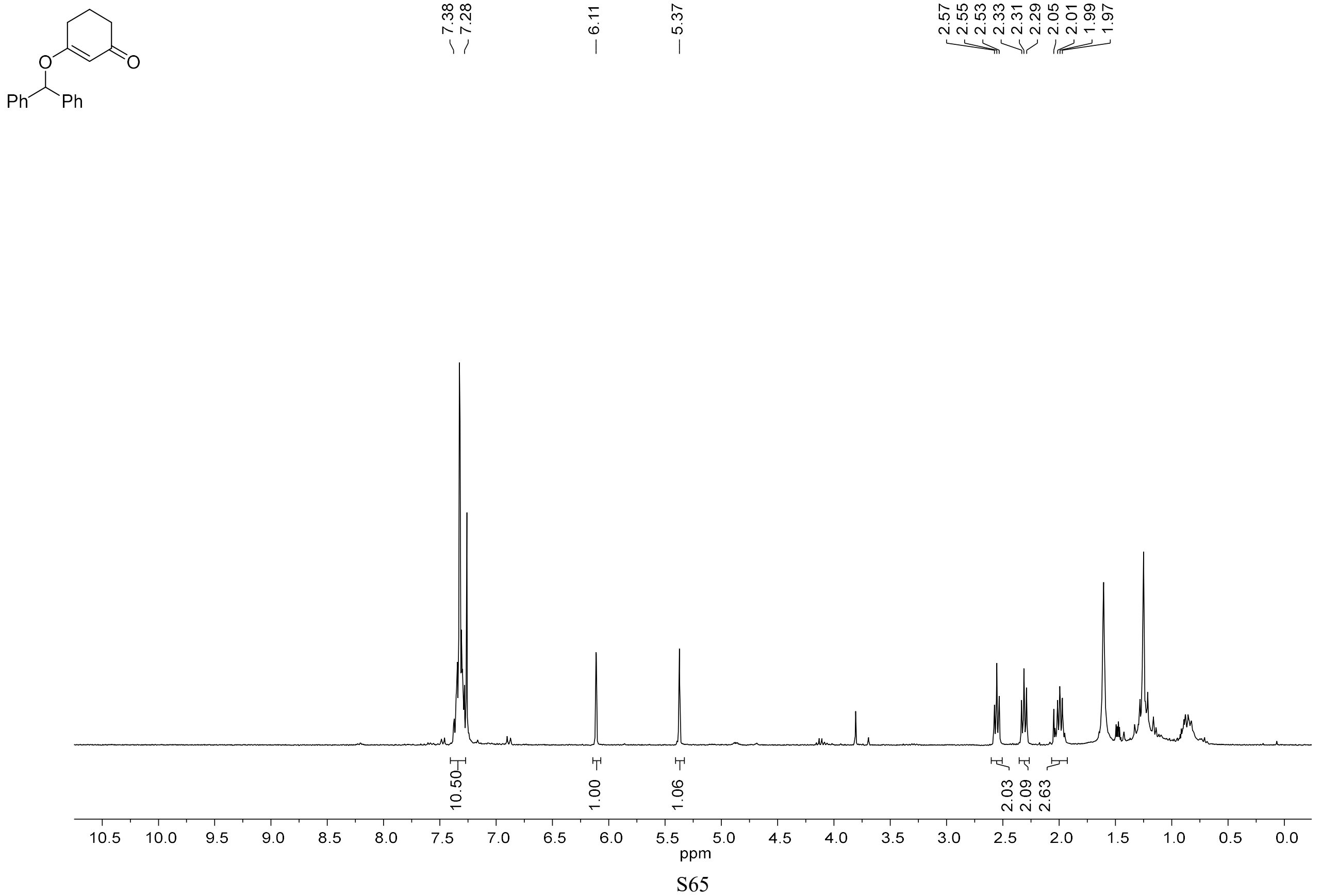
$\mathbf{S 1 7}{ }^{13} \mathrm{C}\left\{{ }^{1} \mathrm{H}\right\} \mathrm{NMR}\left(126 \mathrm{MHz}, \mathrm{CDCl}_{3}\right)$

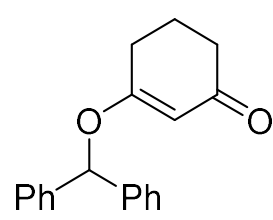

$\mathrm{Ph} \mathrm{Ph}$

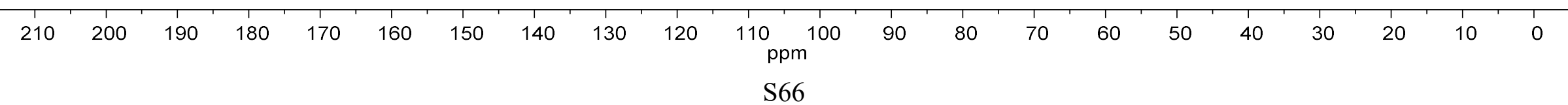


$18{ }^{1} \mathrm{H}$ NMR (400 MHz, $\mathrm{CDCl}_{3}$ )

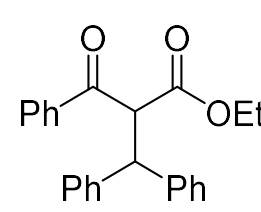

$\mathrm{Ph} P h$

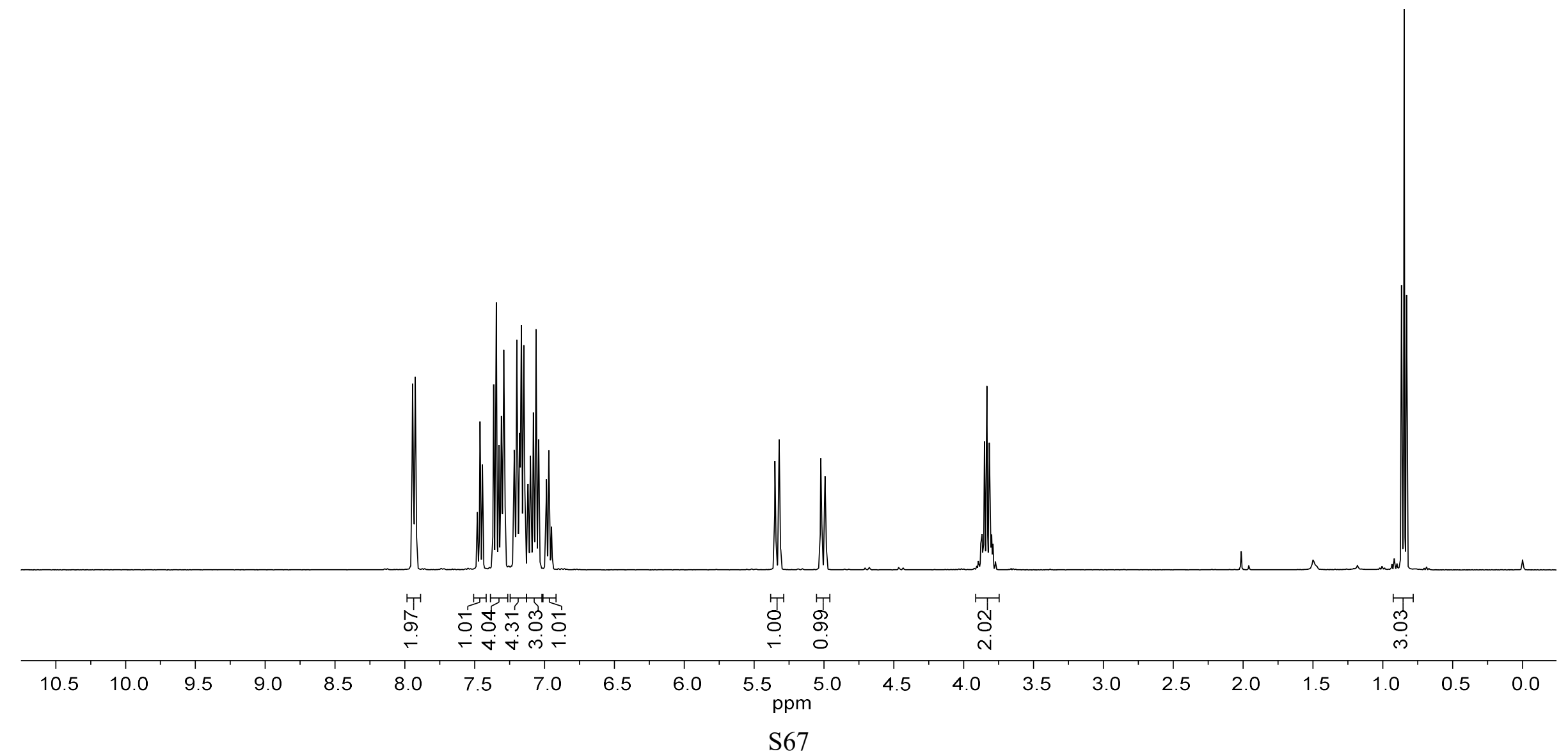


19 (57:43 dr) ${ }^{1} \mathrm{H}$ NMR (300 MHz, $\left.\mathrm{CDCl}_{3}\right)$
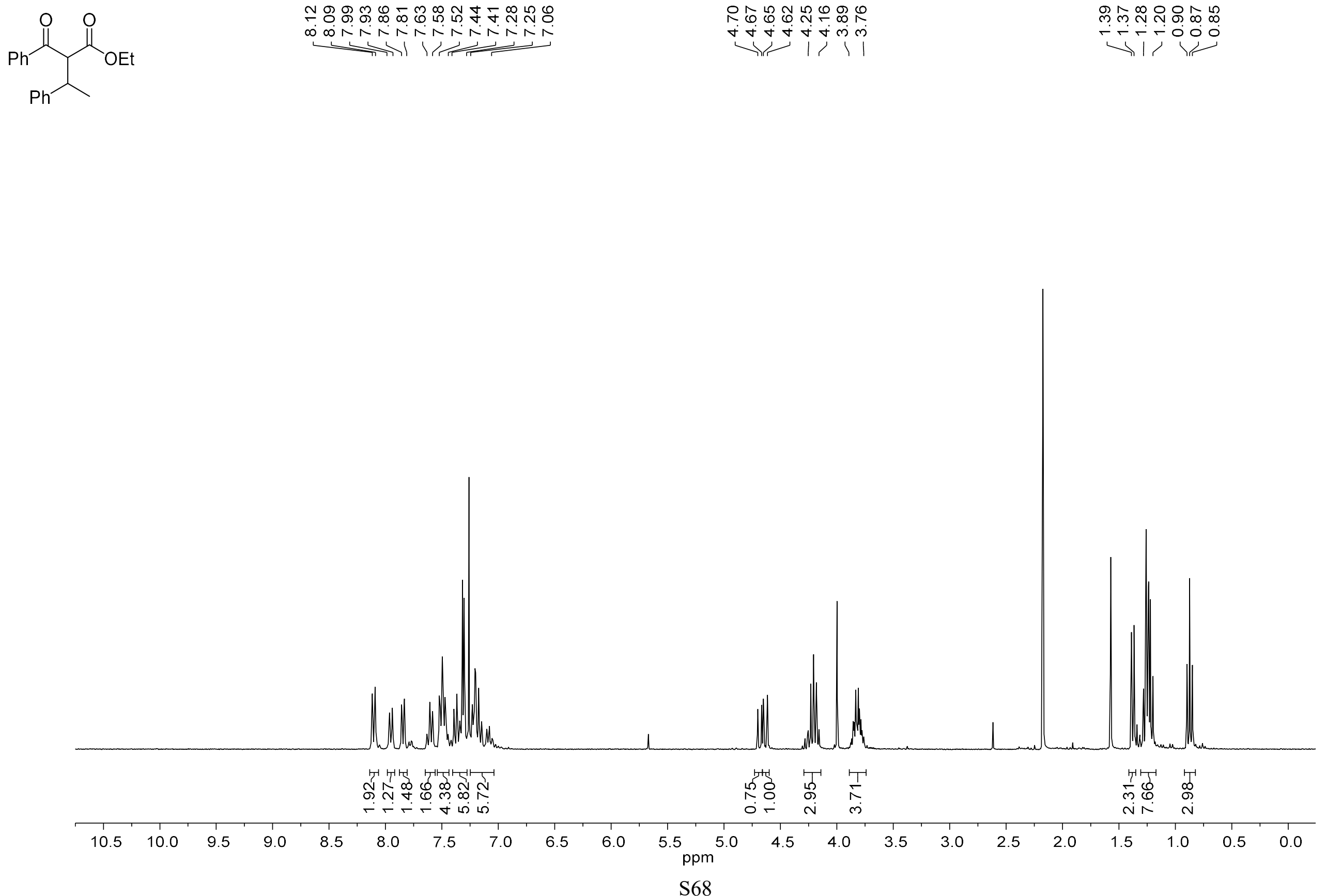
20 (54:46 dr) ${ }^{1} \mathrm{H}$ NMR (300 MHz, $\mathrm{CDCl}_{3}$ )

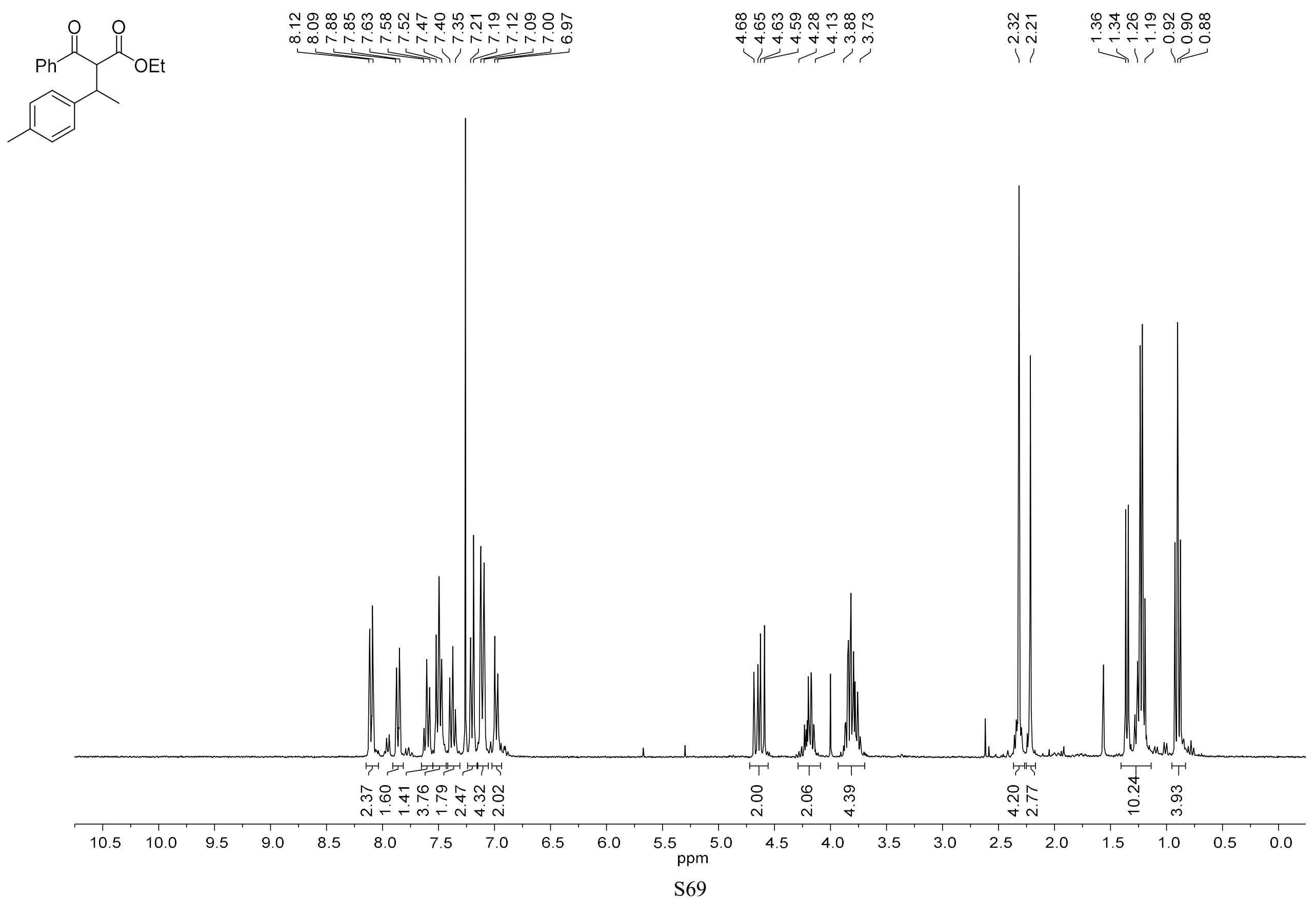


$20(54: 46 \mathrm{dr}){ }^{13} \mathrm{C}\left\{{ }^{1} \mathrm{H}\right\} \mathrm{NMR}\left(126 \mathrm{MHz}, \mathrm{CDCl}_{3}\right)$

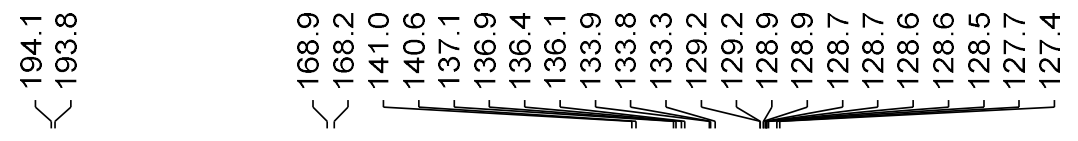

으.

तु $\overline{6} \overline{6}$

일

ㄴ.

(1)

N N
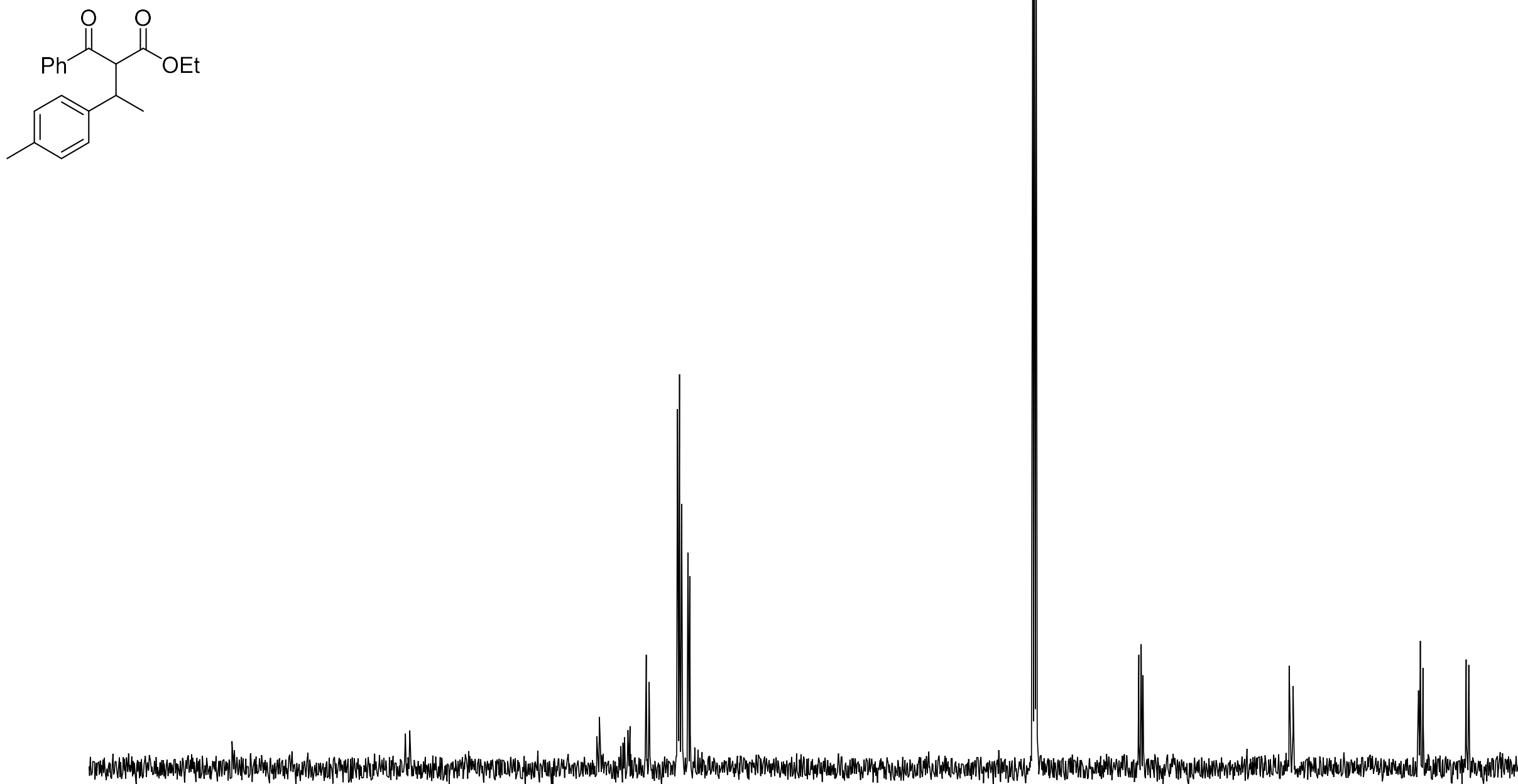

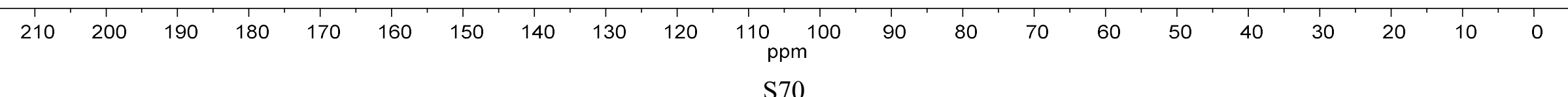


$21(63: 37 \mathrm{dr}){ }^{1} \mathrm{H}$ NMR (400 MHz, $\left.\mathrm{CDCl}_{3}\right)$
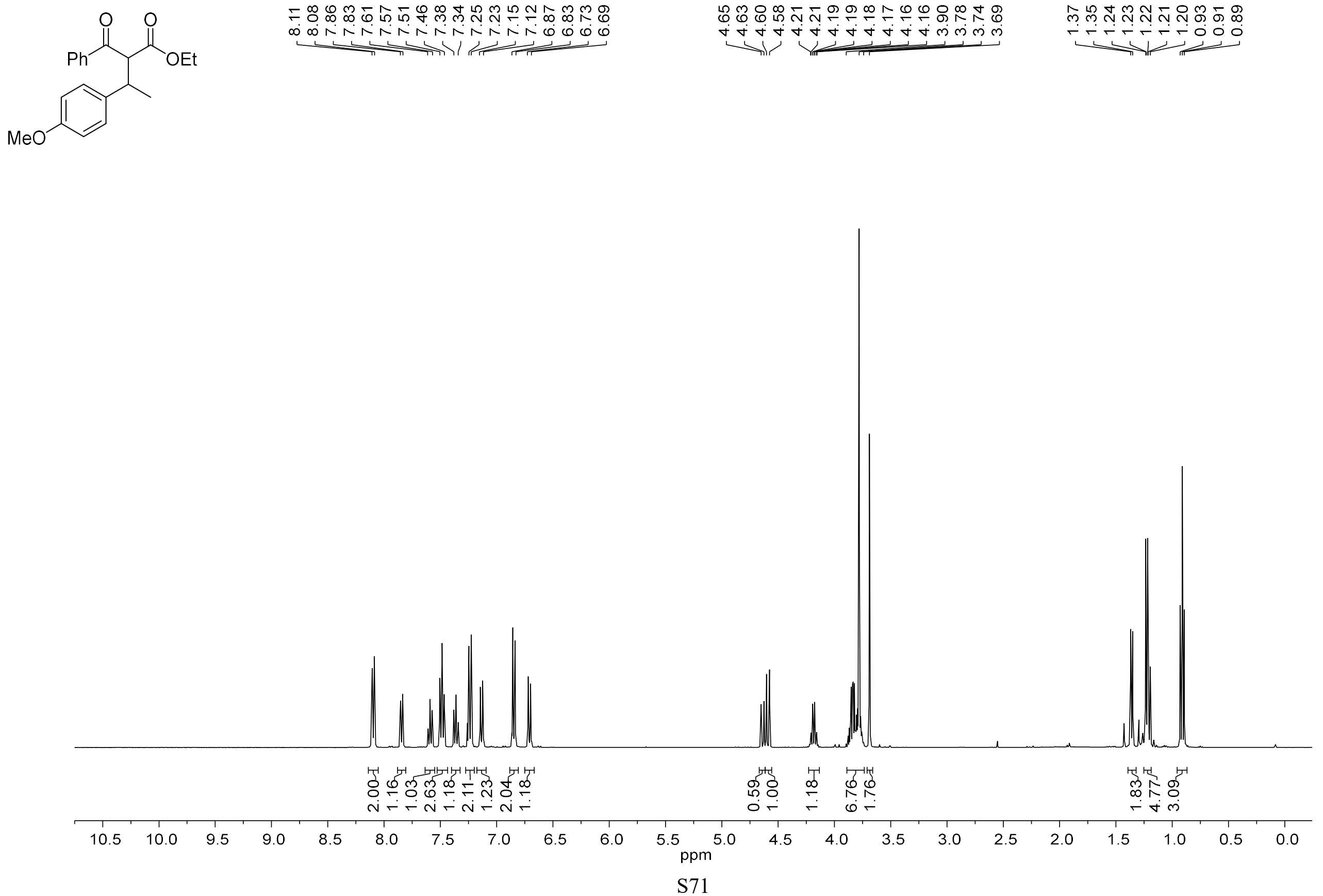
$22(52: 48 \mathrm{dr}){ }^{1} \mathrm{H}$ NMR (300 MHz, $\left.\mathrm{CDCl}_{3}\right)$
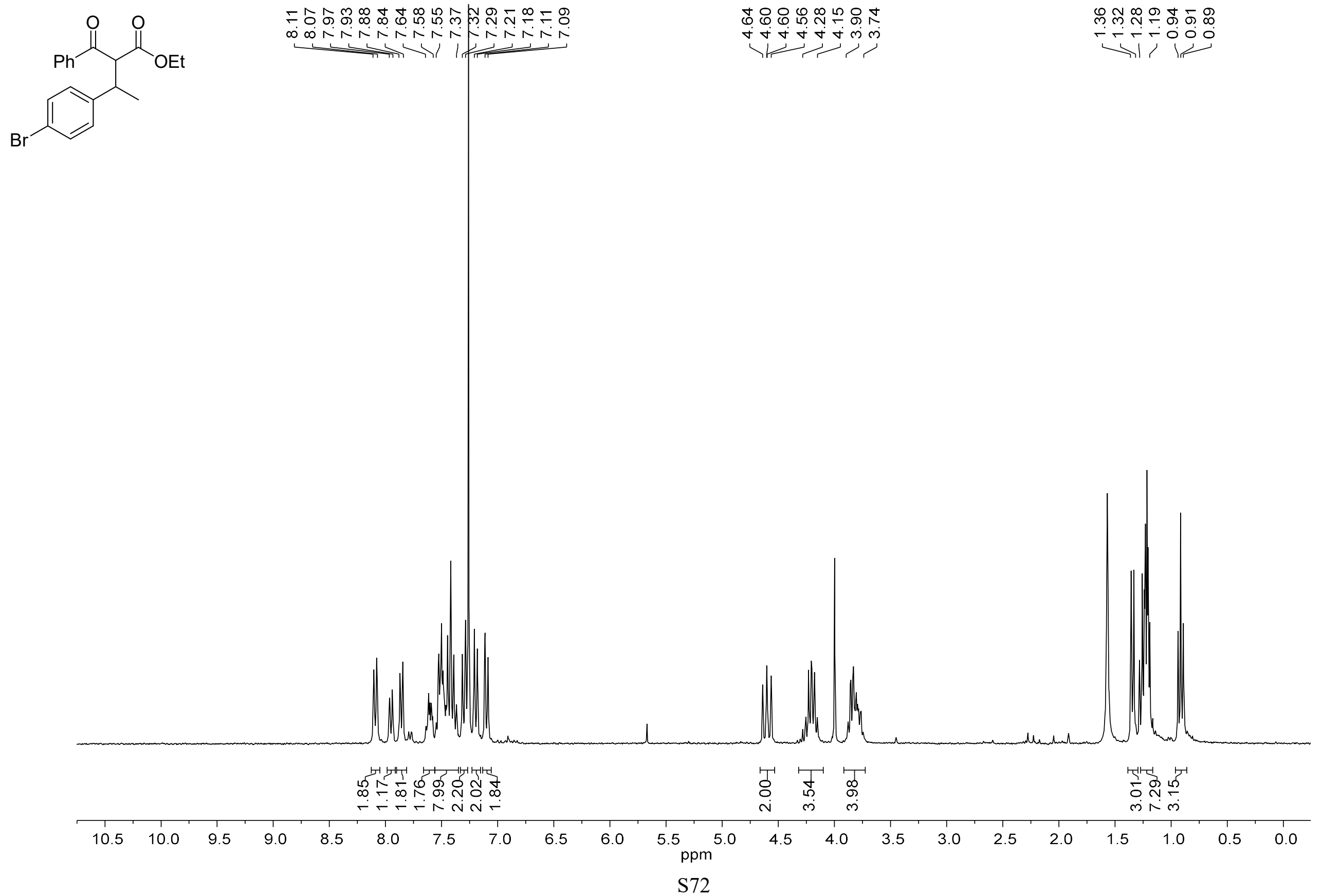
$22(52: 48 \mathrm{dr}){ }^{13} \mathrm{C}\left\{{ }^{1} \mathrm{H}\right\} \mathrm{NMR}\left(126 \mathrm{MHz}, \mathrm{CDCl}_{3}\right)$

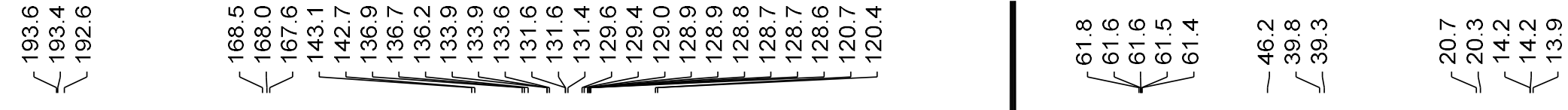

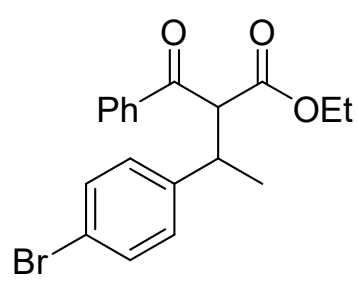

$\mathrm{x}$

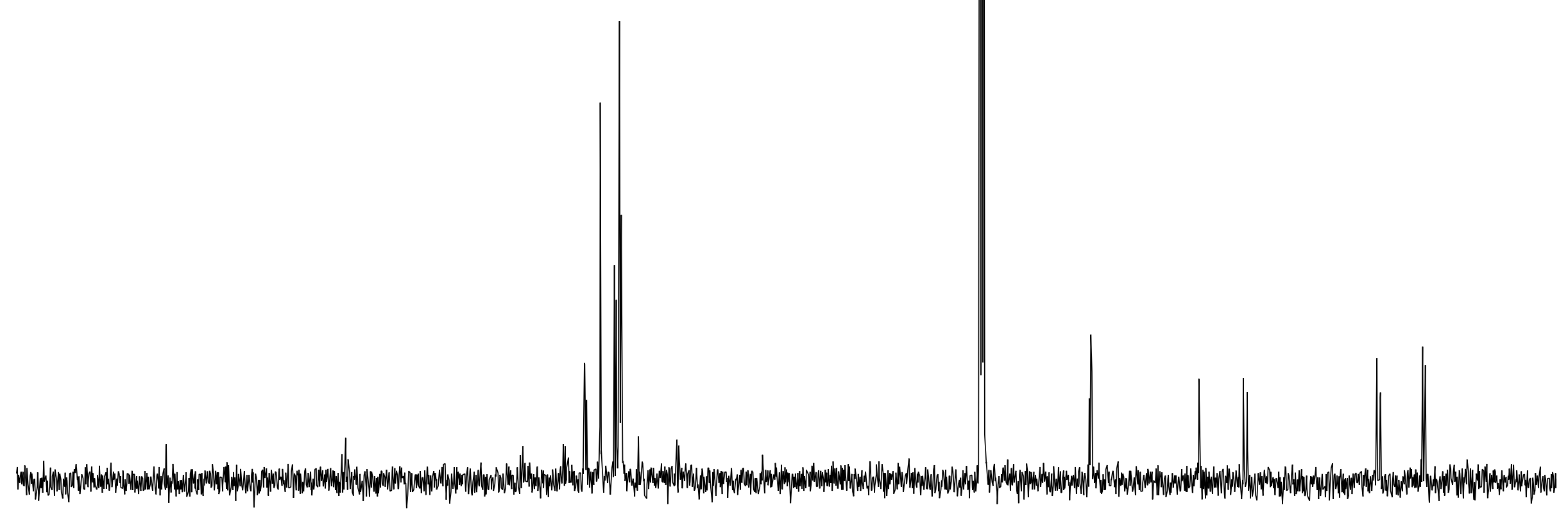


$23{ }^{1} \mathrm{H}$ NMR (300 MHz, $\left.\mathrm{CDCl}_{3}\right)$
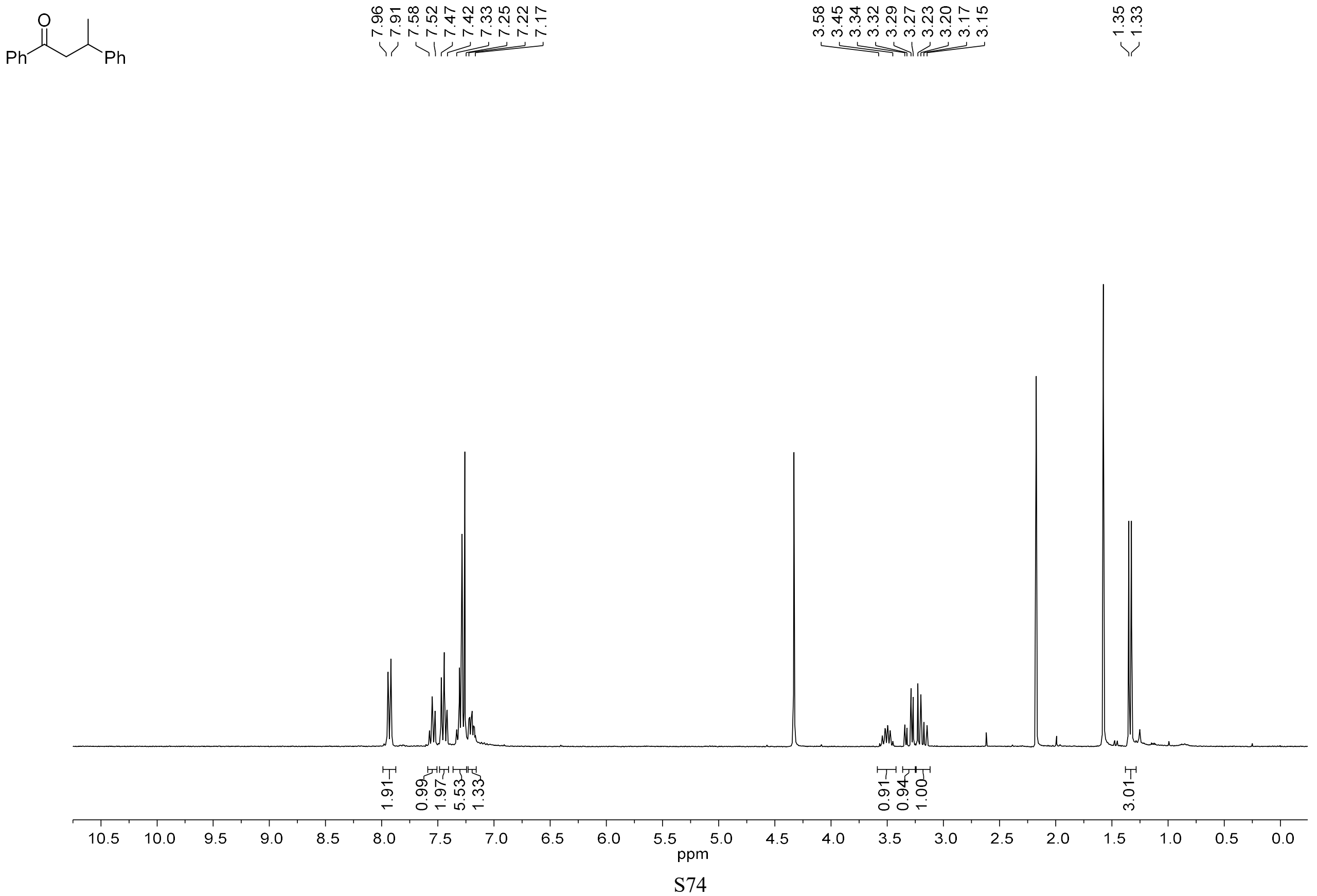


\section{$24{ }^{1} \mathrm{H}$ NMR (300 MHz, $\left.\mathrm{CDCl}_{3}\right)$}
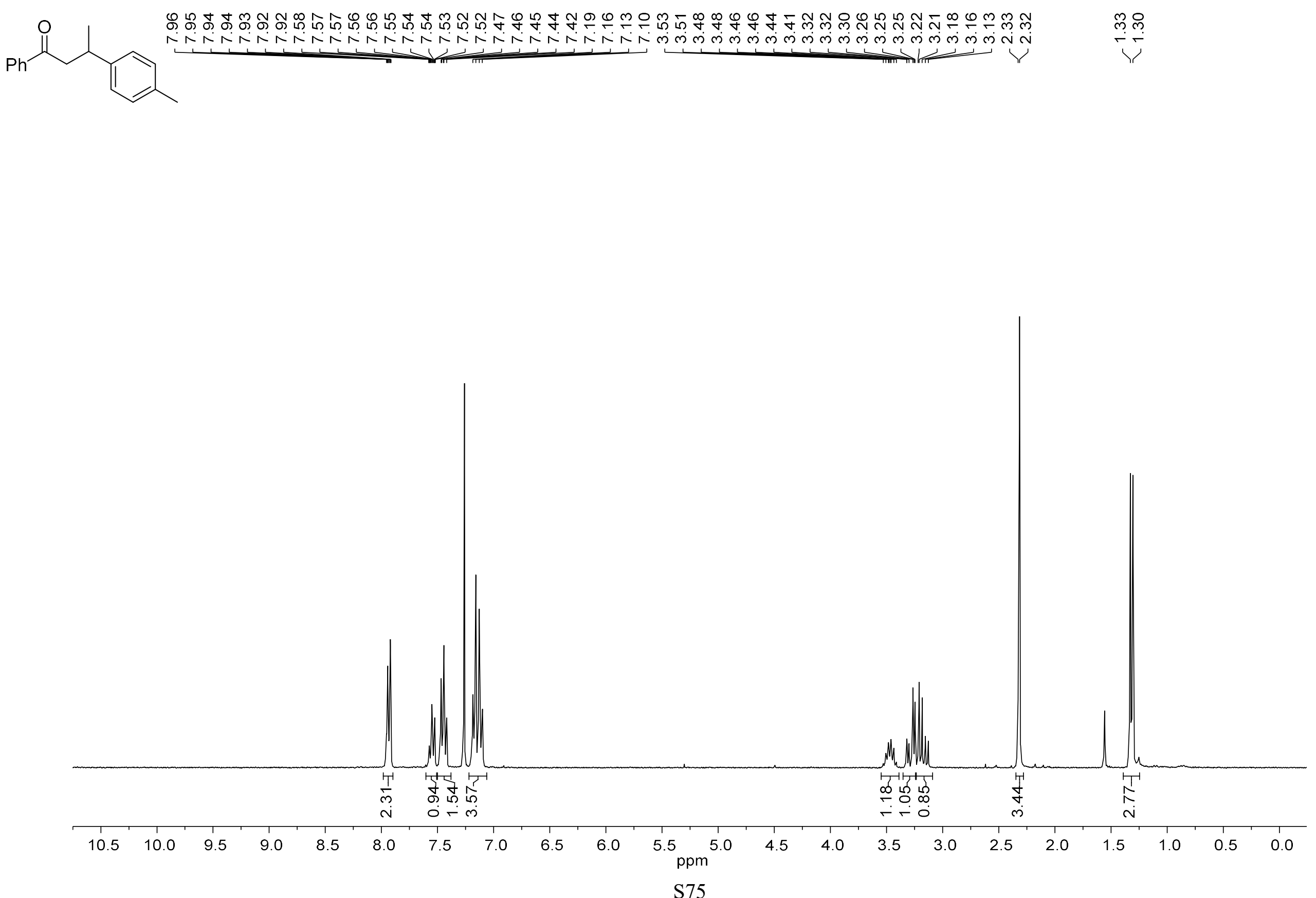
$25{ }^{1} \mathrm{H}$ NMR (300 MHz, $\mathrm{CDCl}_{3}$ )

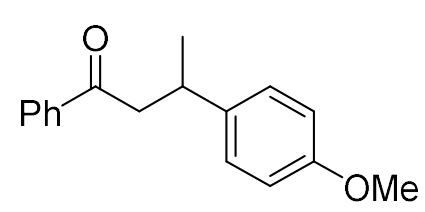

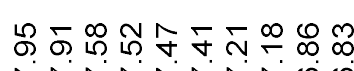

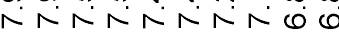

()

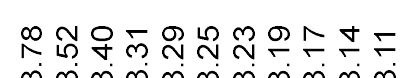

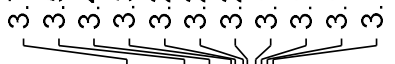

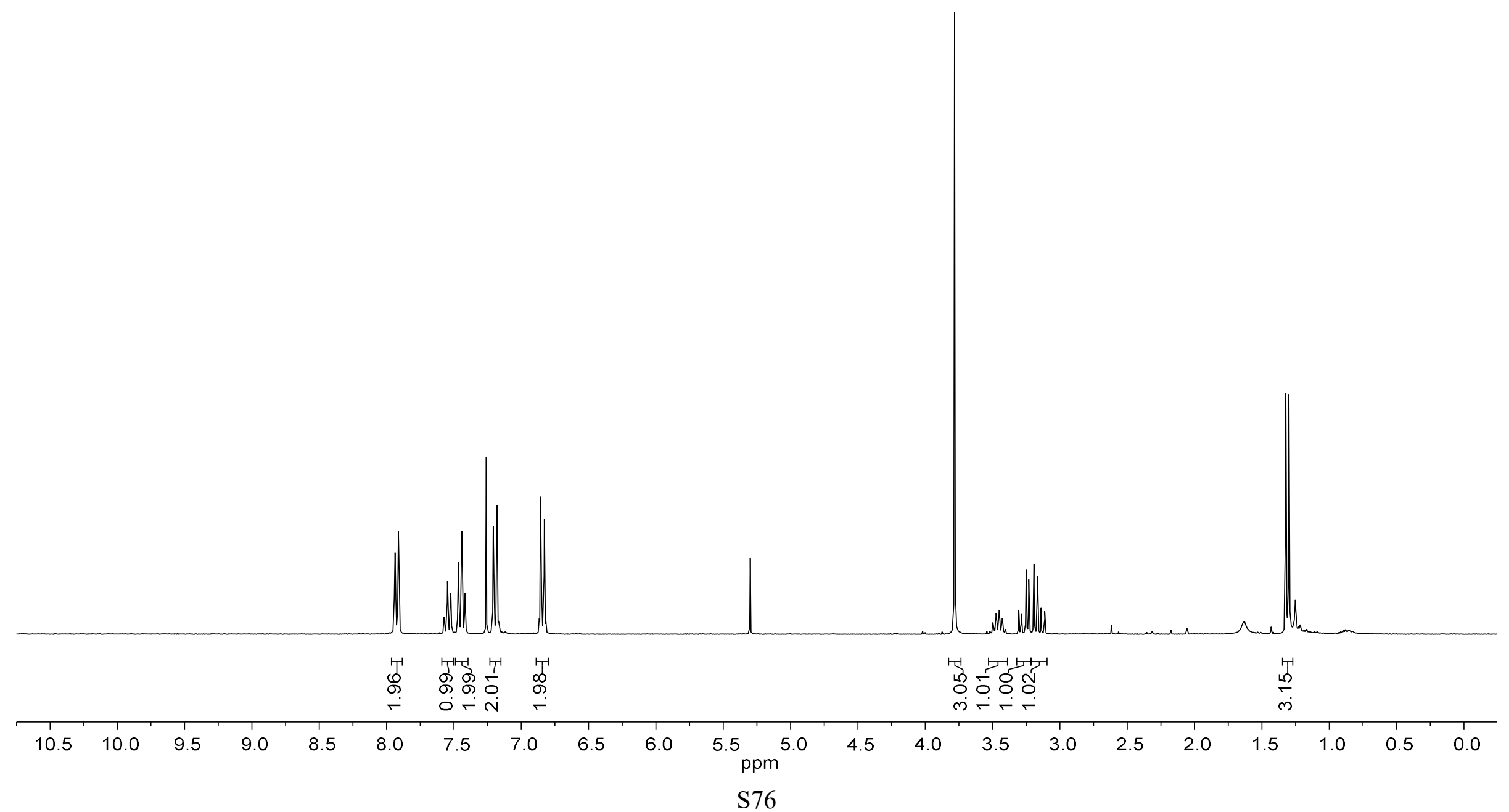


$27{ }^{1} \mathrm{H}$ NMR (300 MHz, $\left.\mathrm{CDCl}_{3}\right)$

\begin{tabular}{|c|c|c|c|c|}
\hline & $\stackrel{\substack{\stackrel{N}{i} \\
i}}{i}$ & 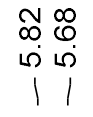 & 움 & 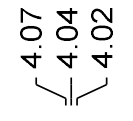 \\
\hline
\end{tabular}

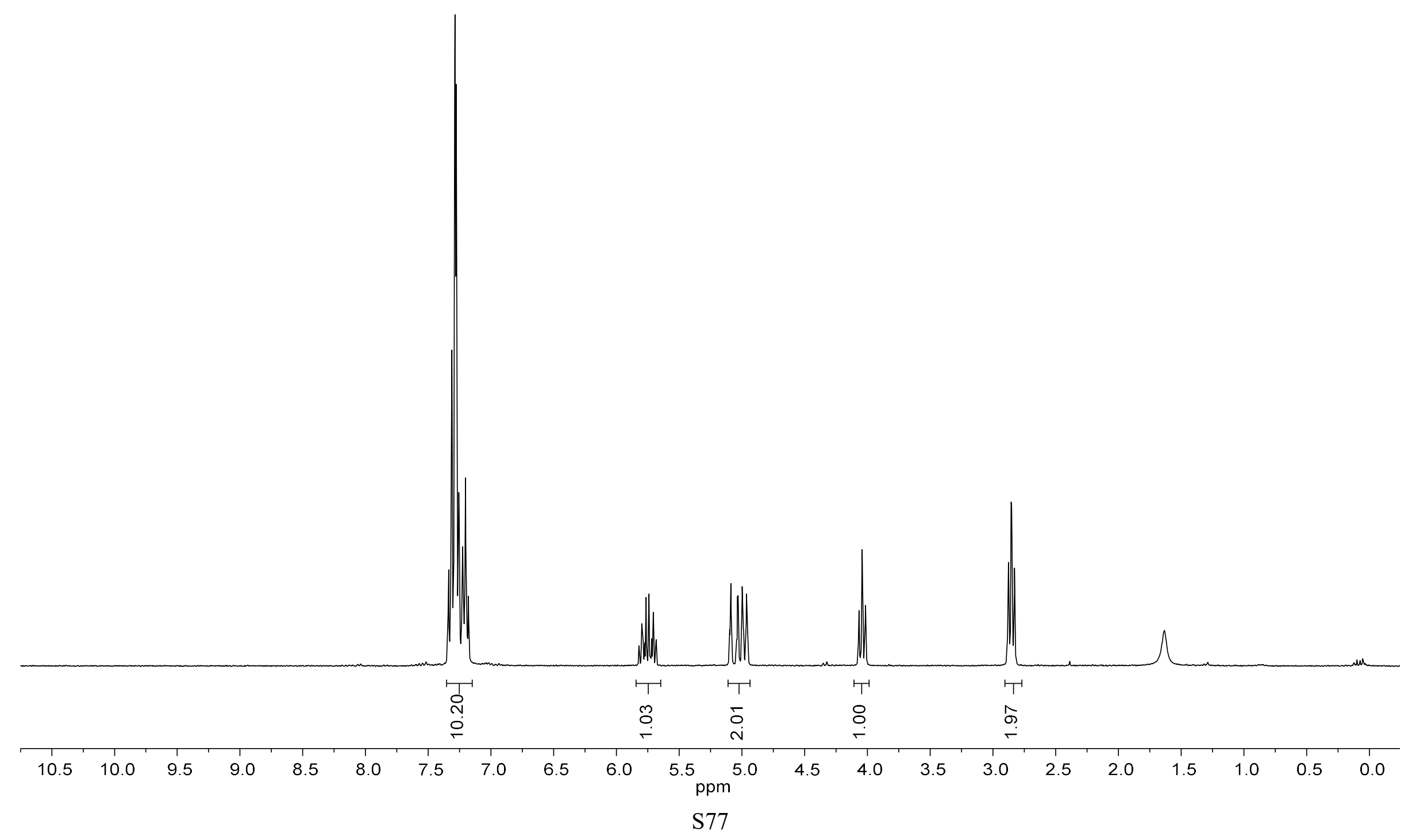


$28{ }^{1} \mathrm{H}$ NMR (300 MHz, $\mathrm{CDCl}_{3}$ )

No

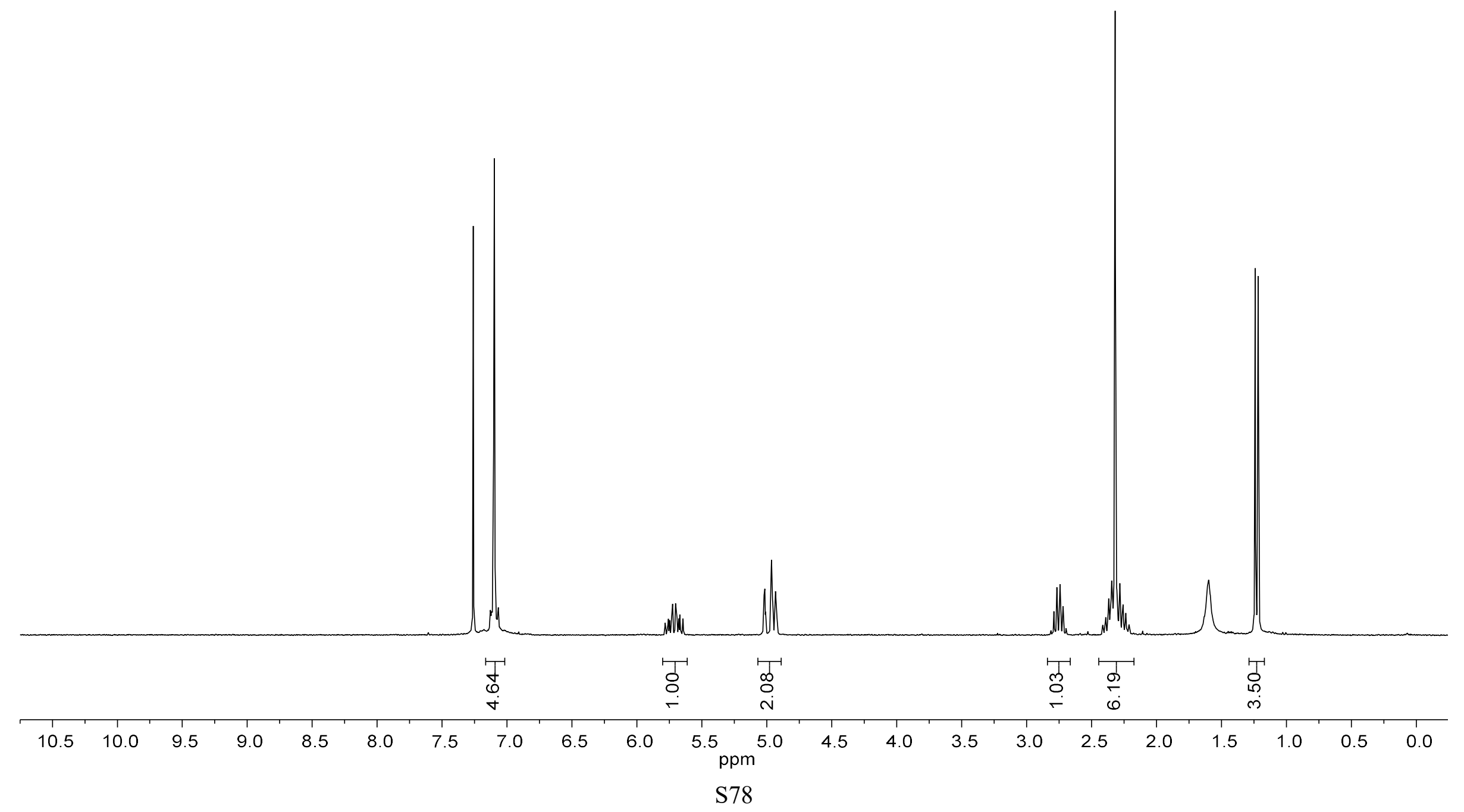


$29{ }^{1} \mathrm{H}$ NMR (300 MHz, $\left.\mathrm{CDCl}_{3}\right)$
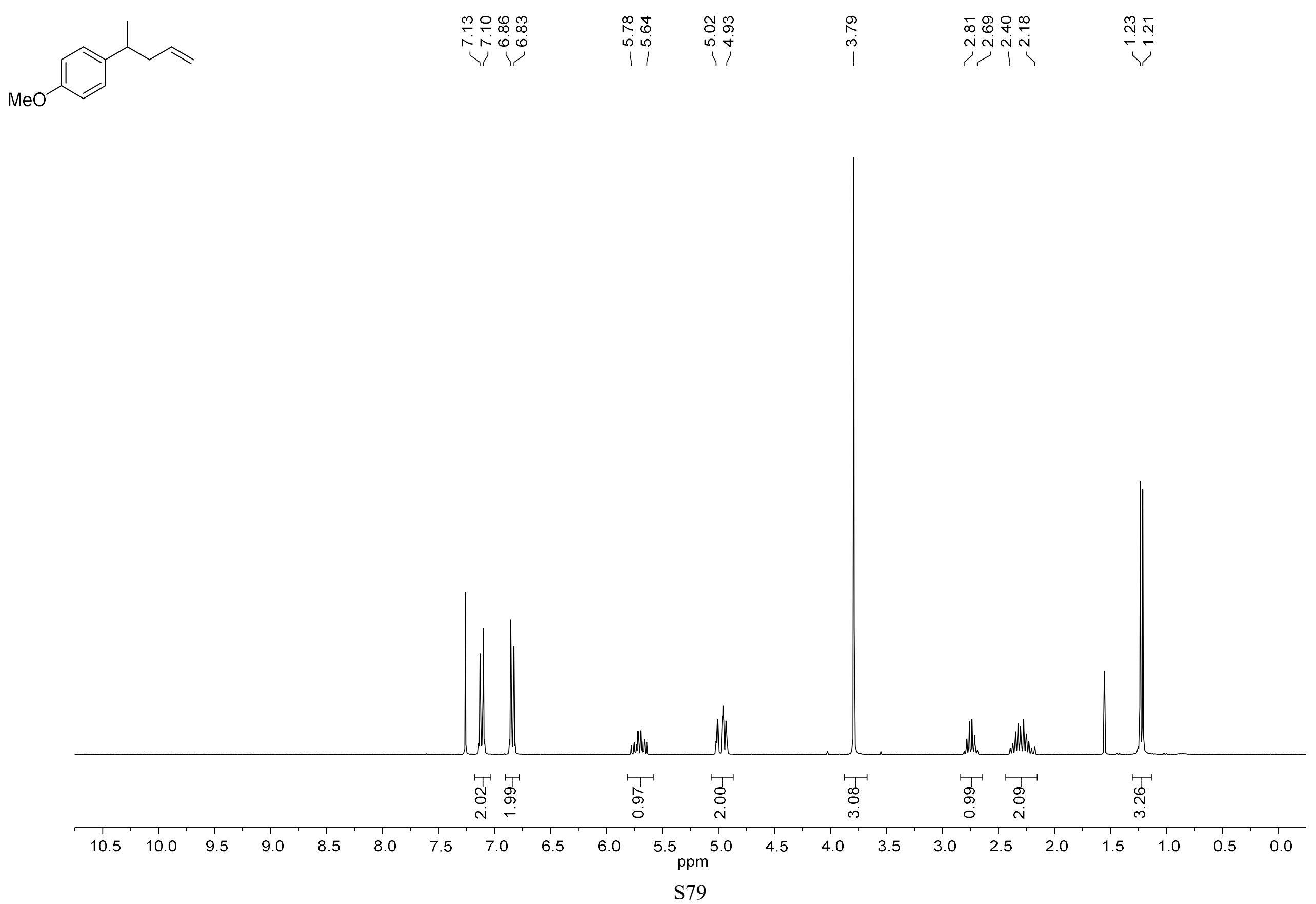
$30{ }^{1} \mathrm{H}$ NMR (300 MHz, $\left.\mathrm{CDCl}_{3}\right)$

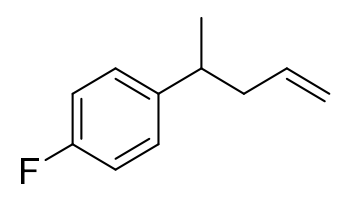

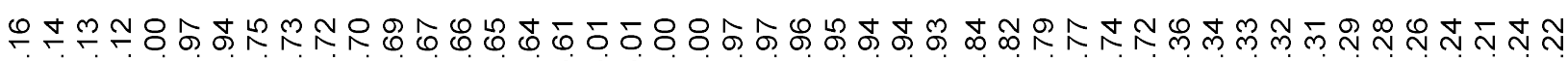

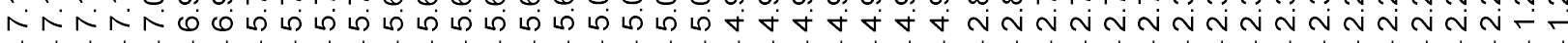
Lin

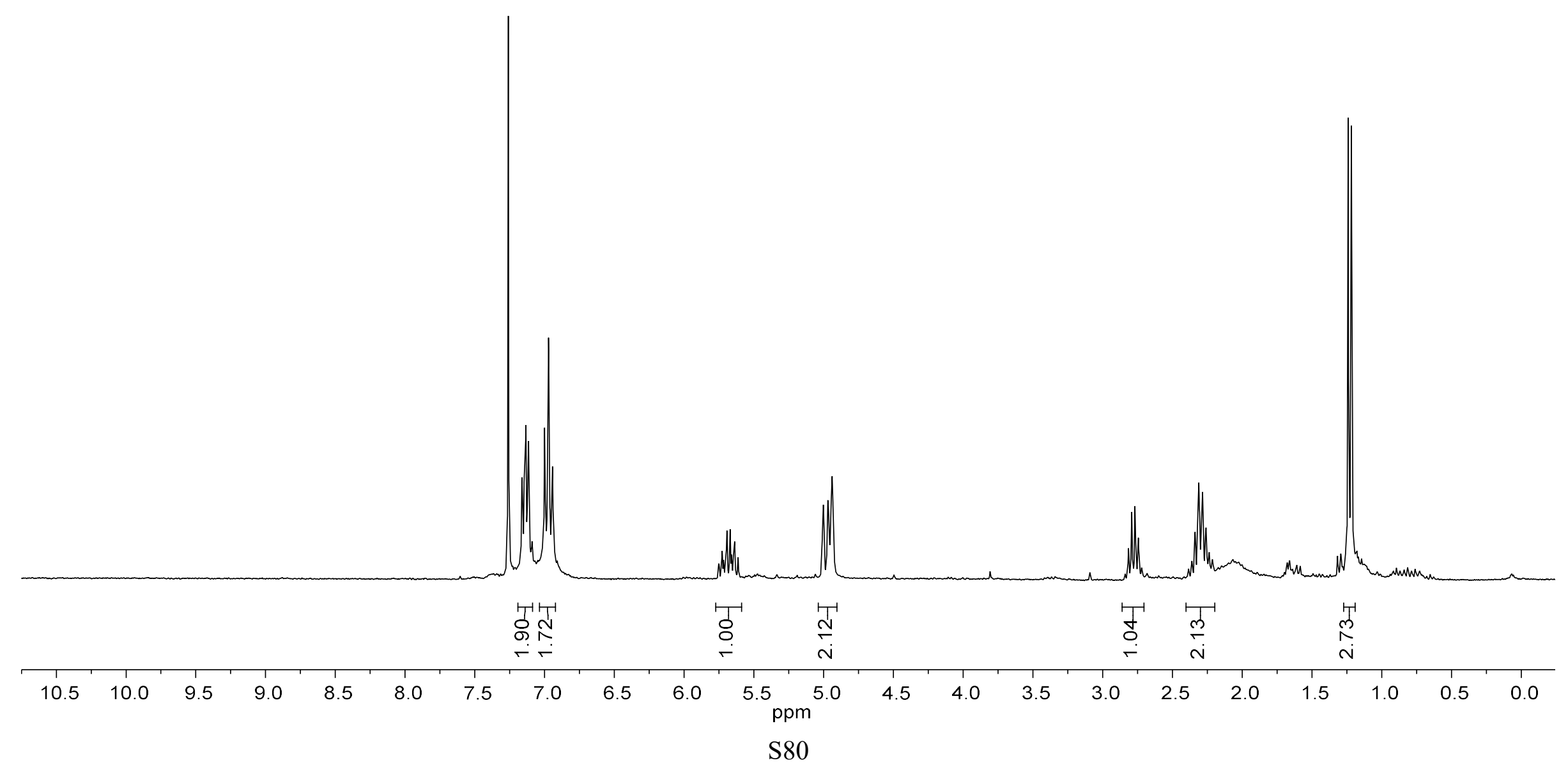


$31{ }^{1} \mathrm{H}$ NMR (300 MHz, $\mathrm{CDCl}_{3}$ )
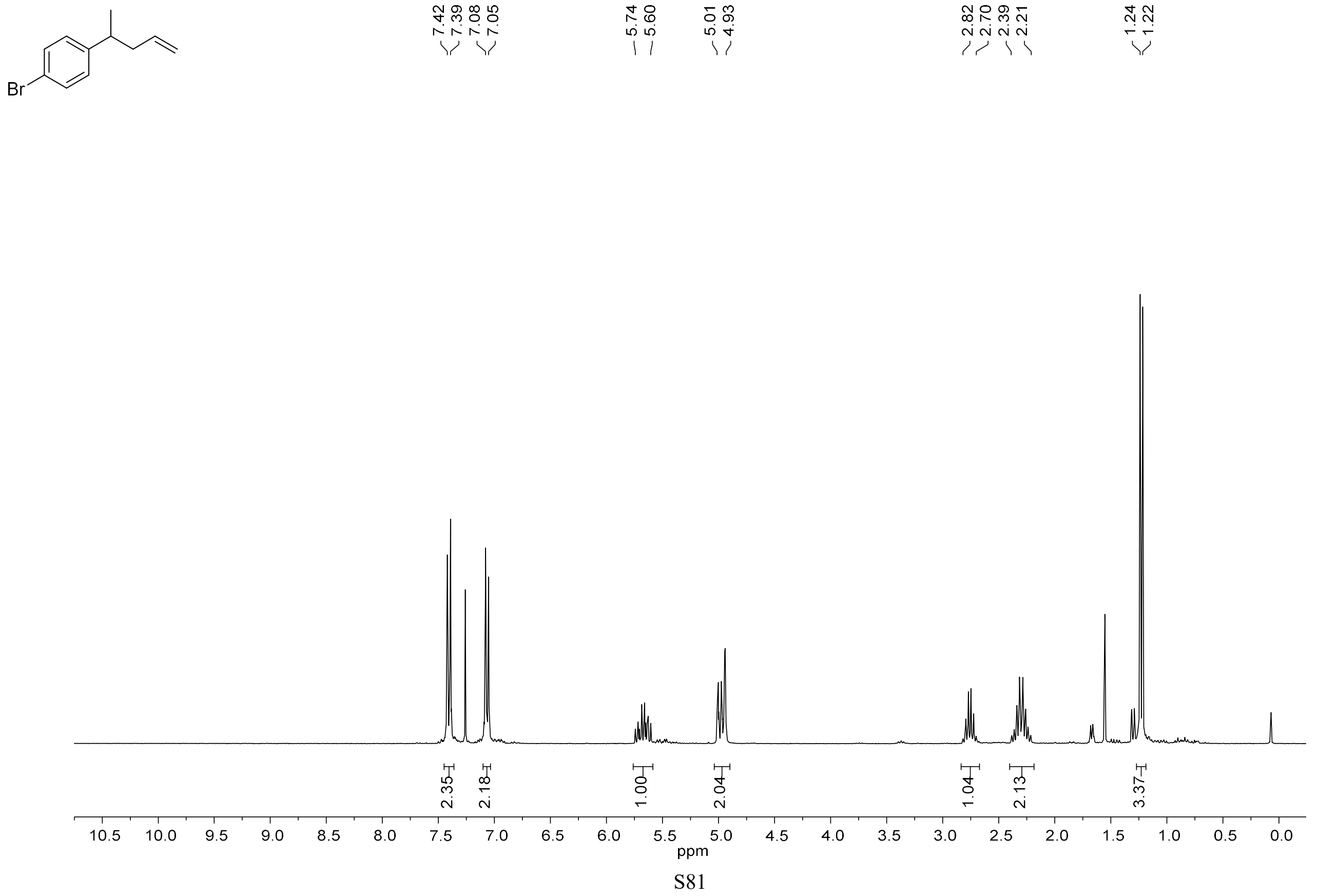
$32{ }^{1} \mathrm{H}$ NMR (300 MHz, $\mathrm{CDCl}_{3}$ )

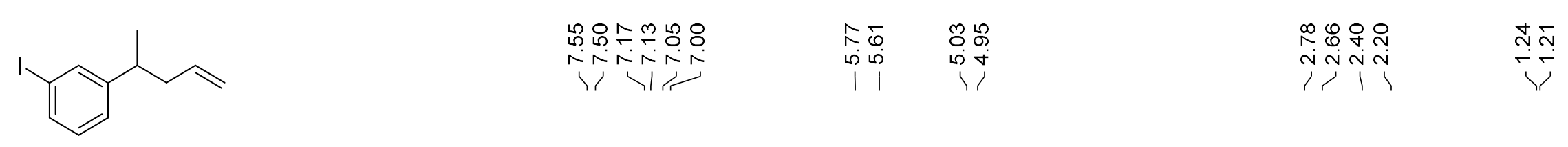

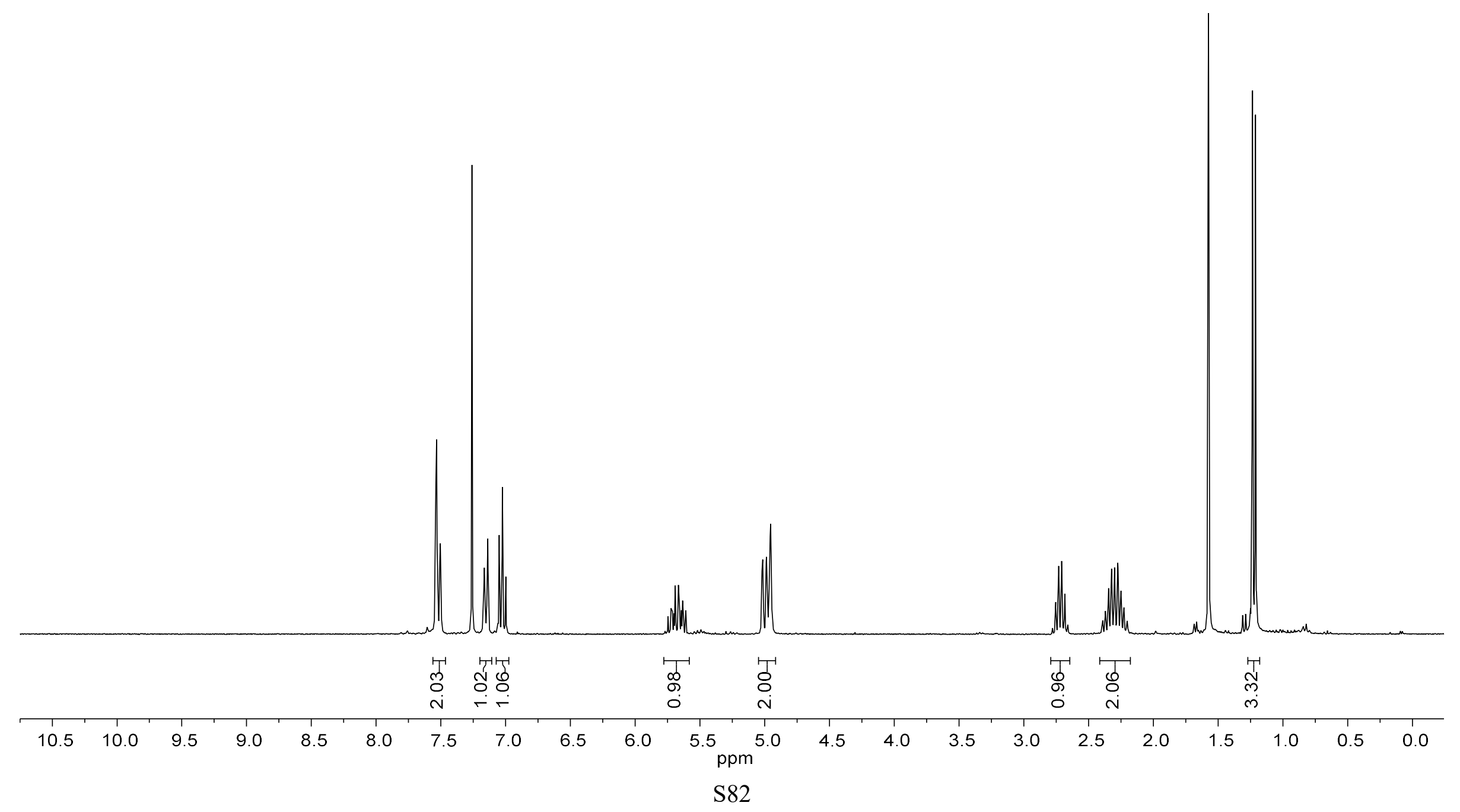


$32{ }^{13} \mathrm{C}\left\{{ }^{1} \mathrm{H}\right\}$ NMR $\left(126 \mathrm{MHz}, \mathrm{CDCl}_{3}\right)$
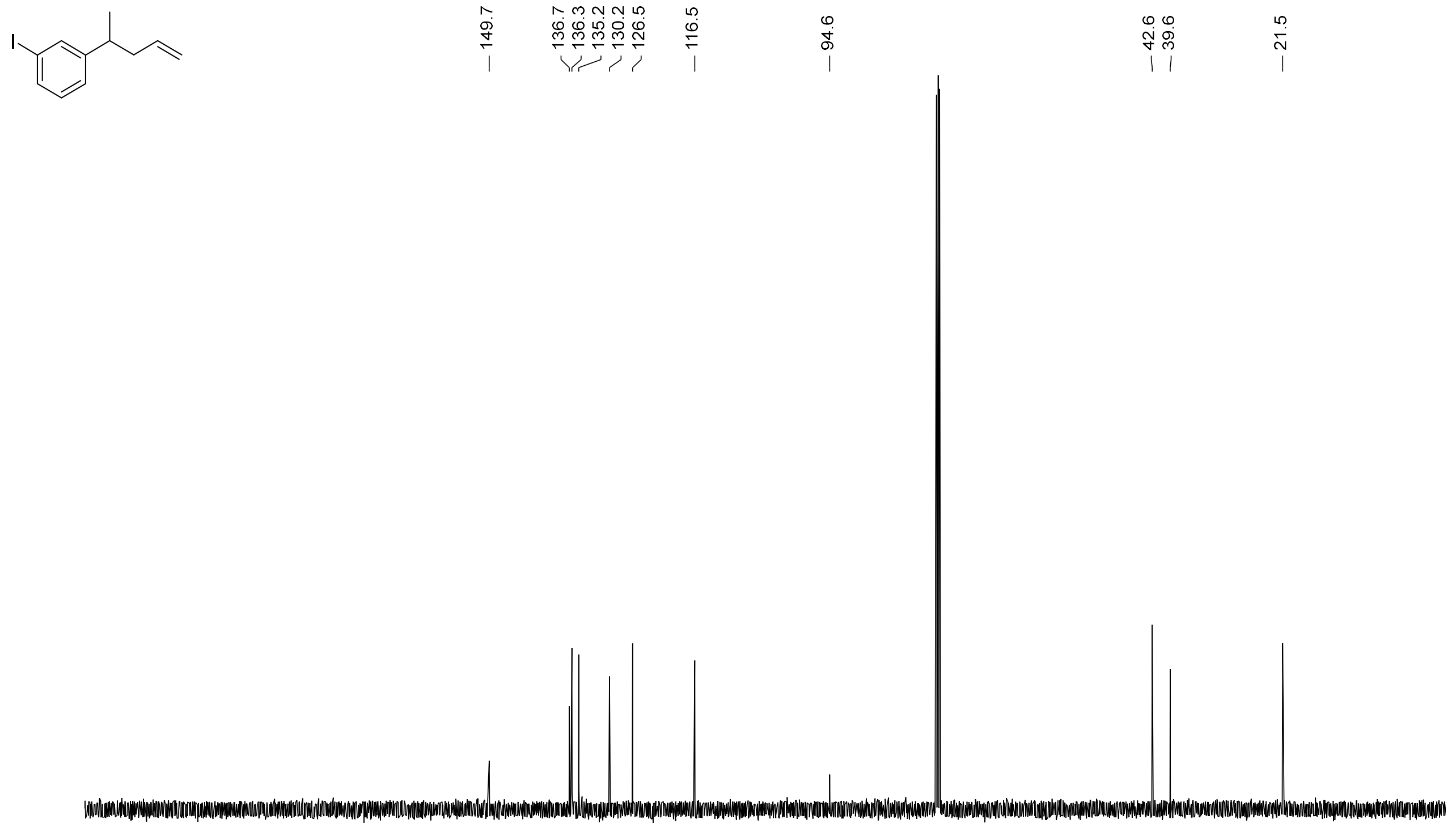

\begin{tabular}{|c|c|c|c|c|c|c|c|c|c|c|c|c|c|c|c|c|c|c|c|c|}
\hline 210 & 200 & 190 & 180 & 170 & 160 & 150 & 140 & 130 & 120 & ${ }_{\mathrm{ppm}}^{110} 100$ & 90 & 80 & 70 & 60 & 50 & 40 & 30 & 20 & 10 & 0 \\
\hline
\end{tabular}


$33{ }^{1} \mathrm{H}$ NMR (300 MHz, $\left.\mathrm{CDCl}_{3}\right)$
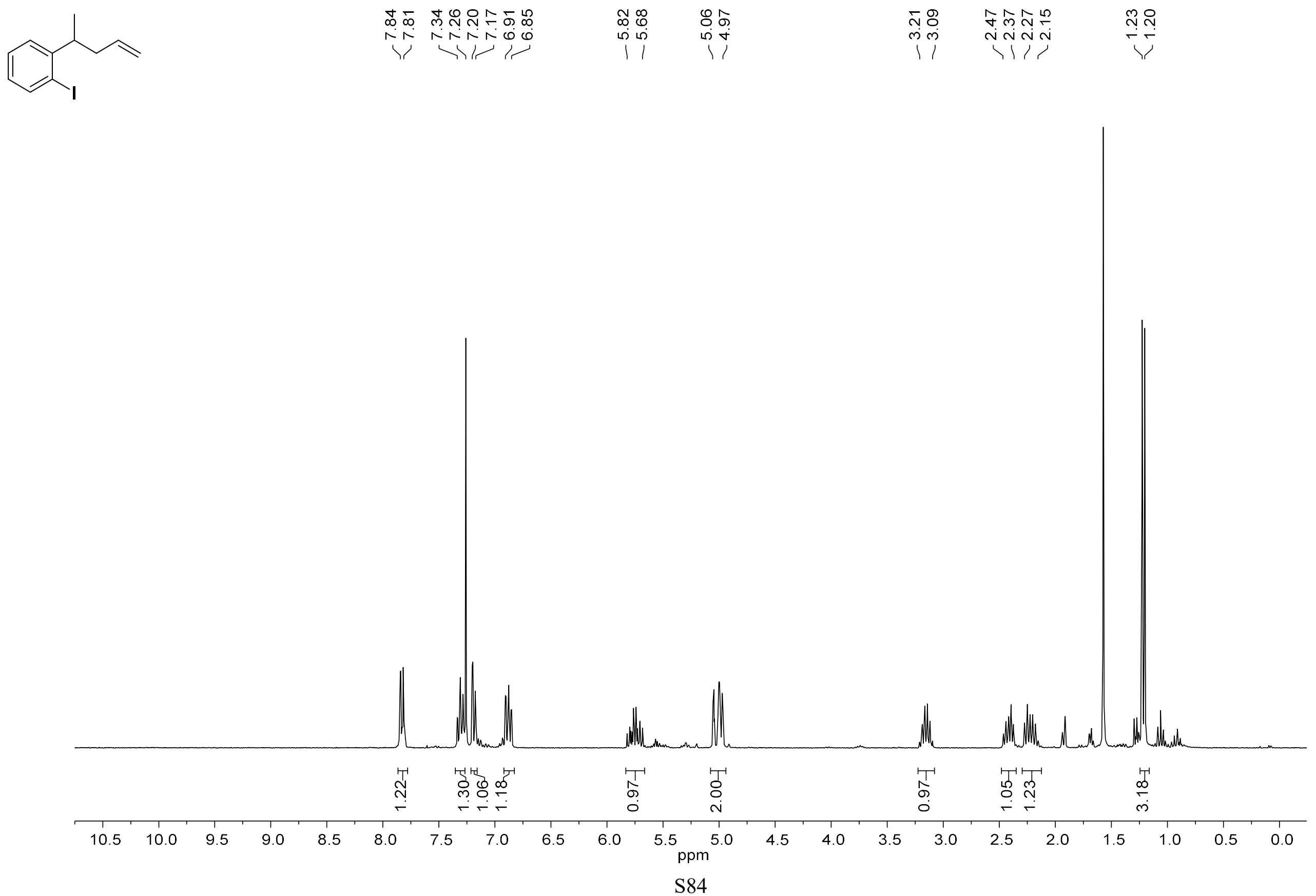
$33{ }^{13} \mathrm{C}\left\{{ }^{1} \mathrm{H}\right\}$ NMR (126 MHz, $\left.\mathrm{CDCl}_{3}\right)$

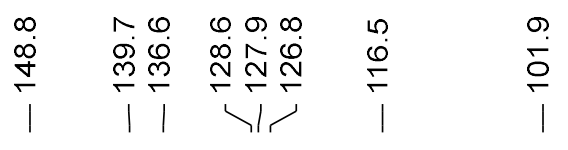

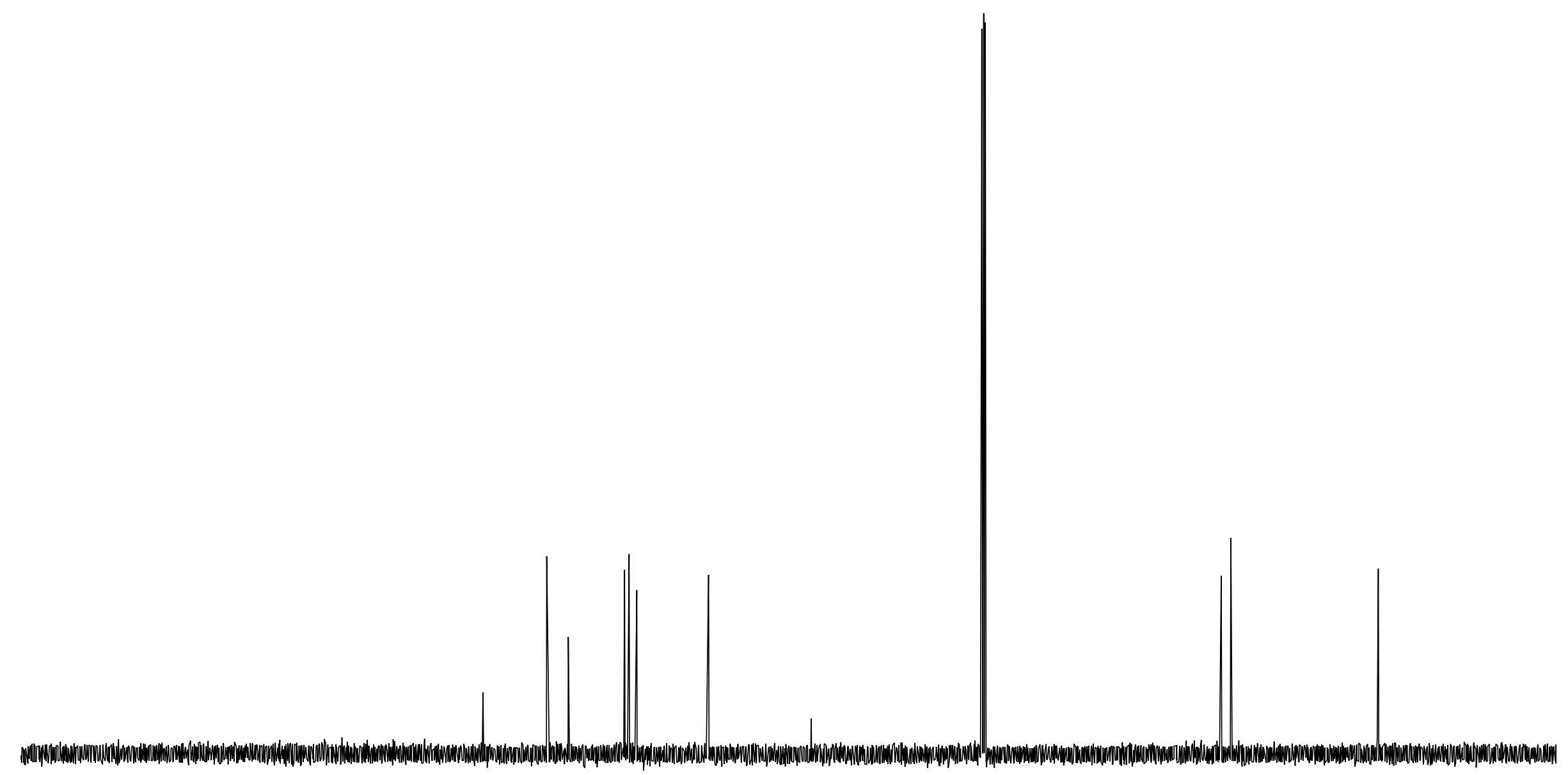

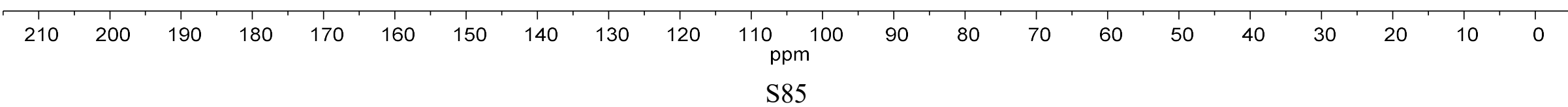


$34{ }^{1} \mathrm{H}$ NMR (300 MHz, $\mathrm{CDCl}_{3}$ )

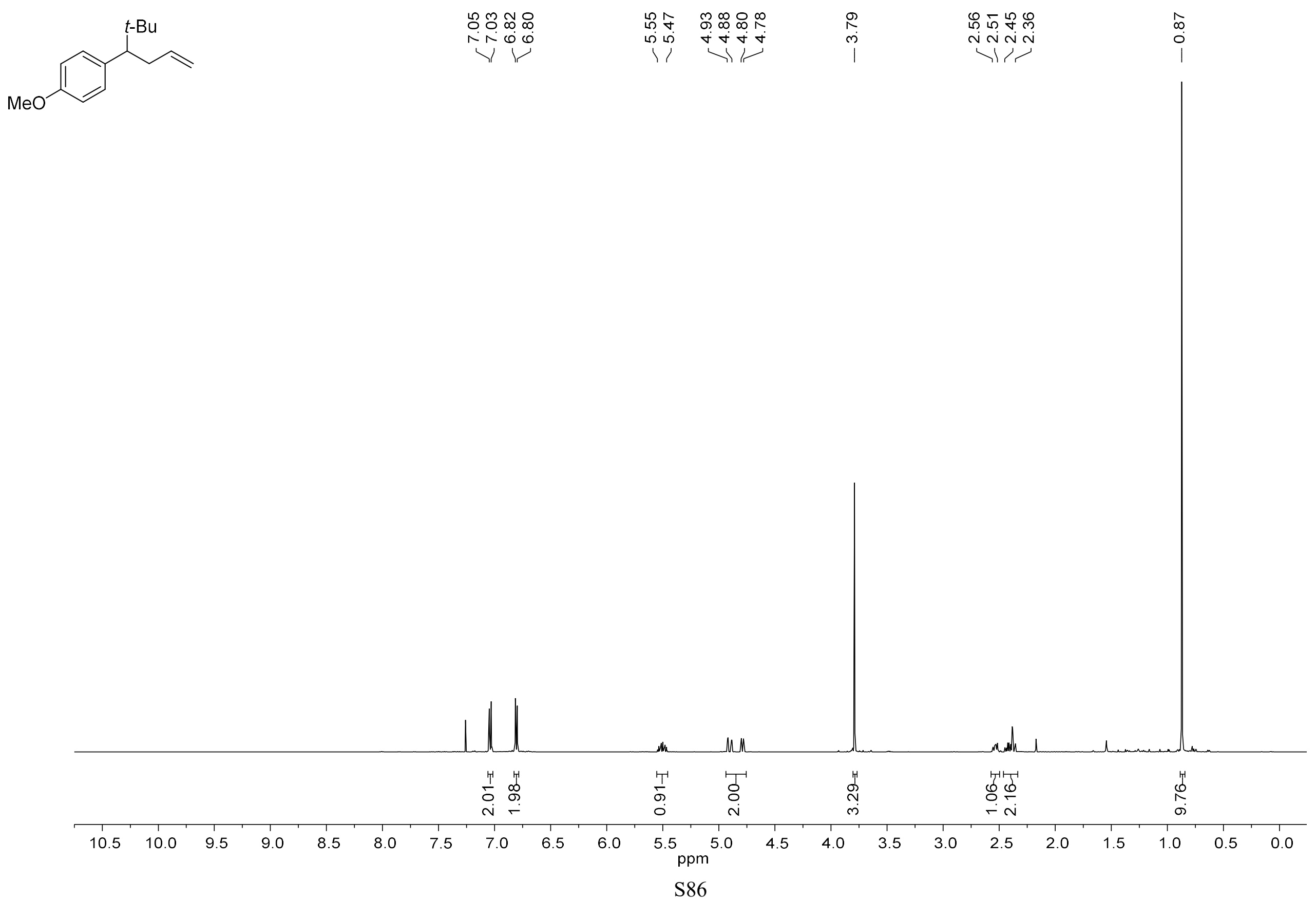


$34{ }^{13} \mathrm{C}\left\{{ }^{1} \mathrm{H}\right\}$ NMR (126 MHz, $\left.\mathrm{CDCl}_{3}\right)$

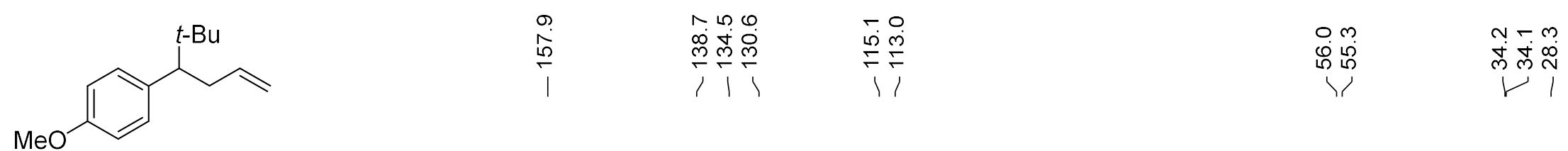

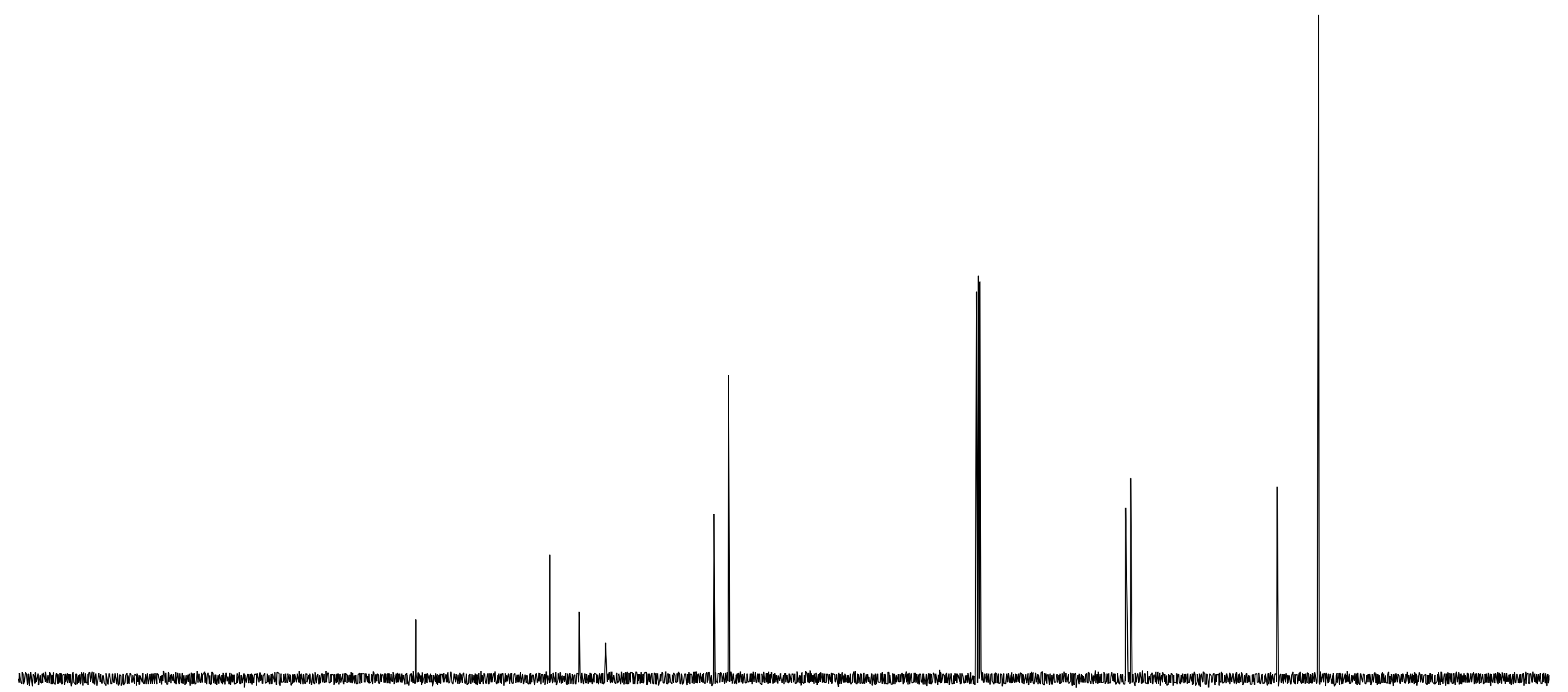

\begin{tabular}{|c|c|c|c|c|c|c|c|c|c|c|c|c|c|c|c|c|c|c|c|c|}
\hline 210 & 200 & 190 & 180 & 170 & 160 & 150 & 140 & 130 & 120 & $\mathrm{ppm}^{110} 100$ & 90 & 80 & 70 & 60 & 50 & 40 & 30 & 20 & 10 & 0 \\
\hline
\end{tabular}


$35{ }^{1} \mathrm{H}$ NMR (300 MHz, $\mathrm{CDCl}_{3}$ )
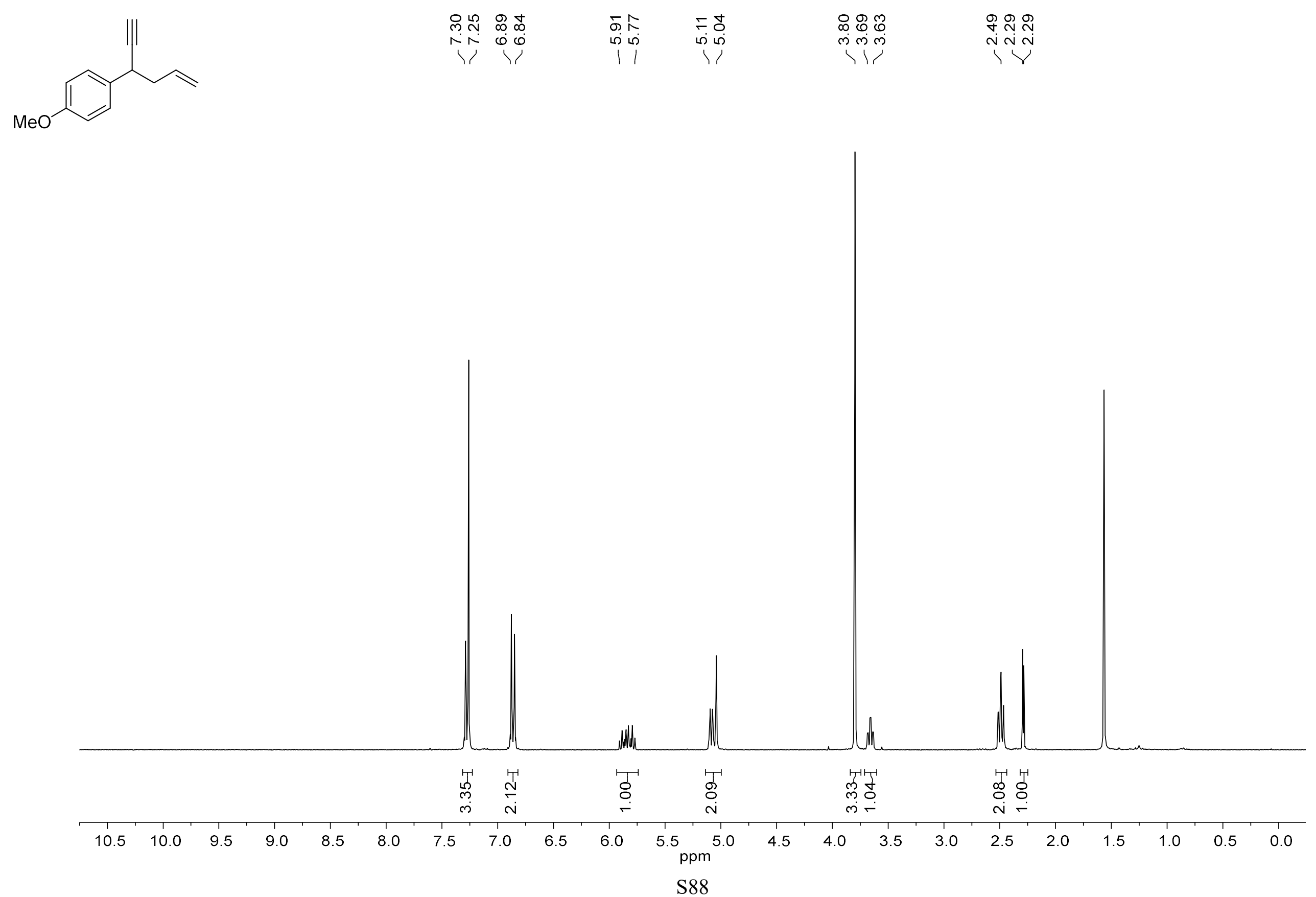
$36{ }^{1} \mathrm{H}$ NMR (300 MHz, $\mathrm{CDCl}_{3}$ )

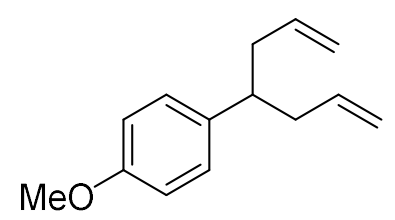

\begin{tabular}{|c|c|c|c|}
\hline 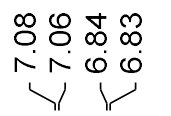 & 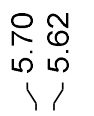 & 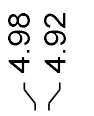 & $\begin{array}{l}9 \\
p \\
m \\
1\end{array}$ \\
\hline
\end{tabular}

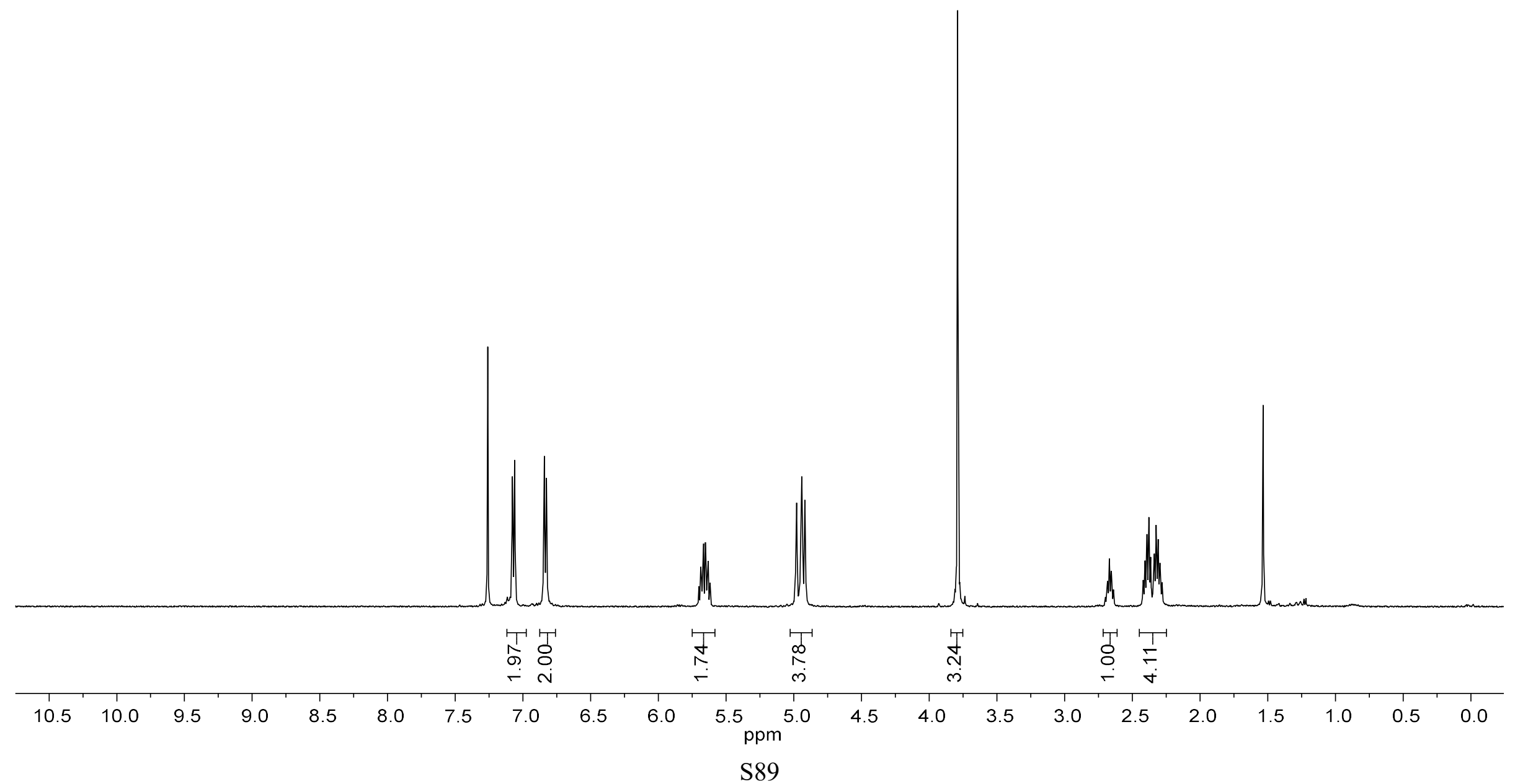


$37{ }^{1} \mathrm{H}$ NMR (300 MHz, $\mathrm{CDCl}_{3}$ )

(N)

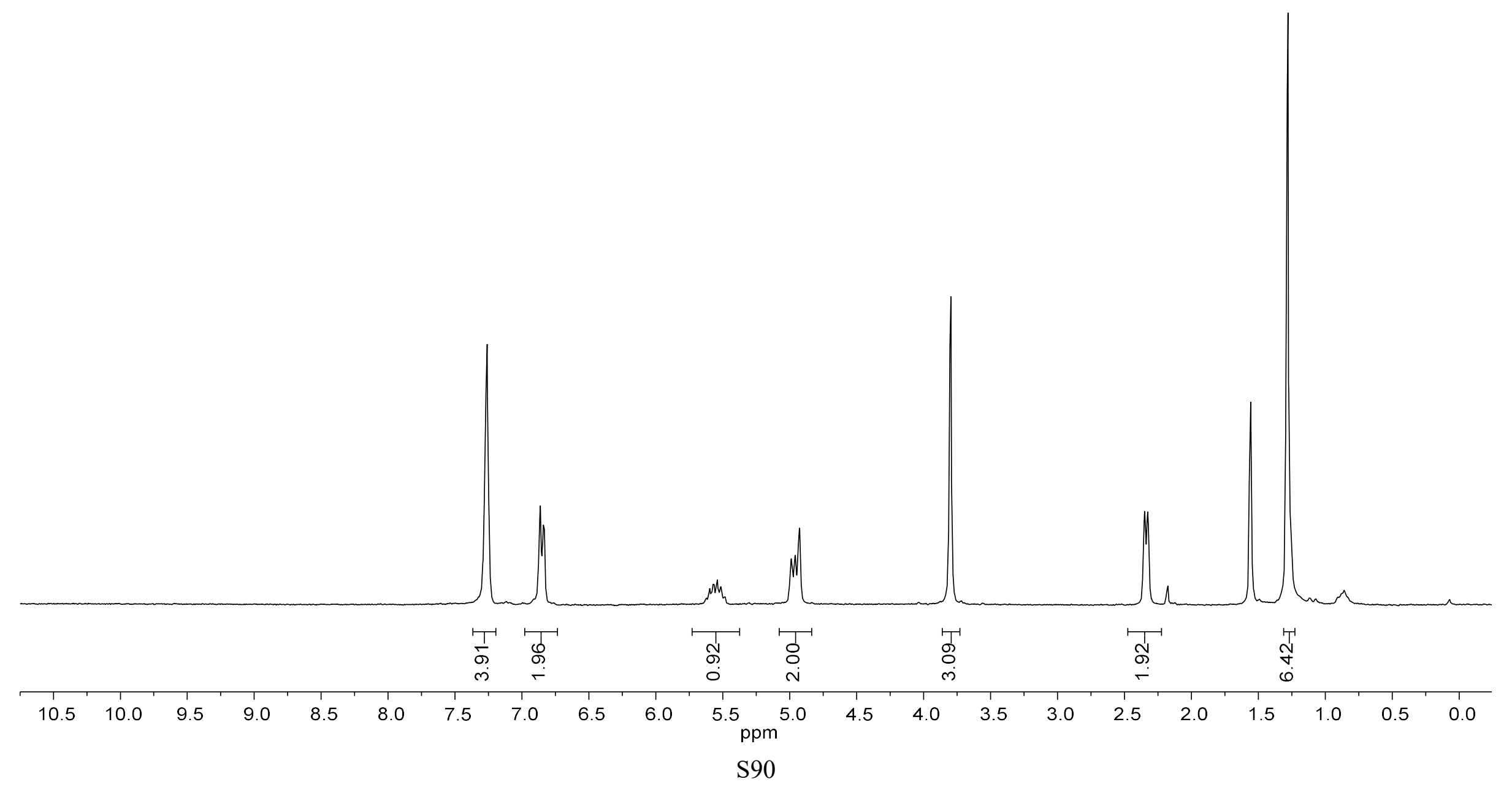


38 (64:36 dr) ${ }^{1} \mathrm{H}$ NMR (300 MHz, $\left.\mathrm{CDCl}_{3}\right)$
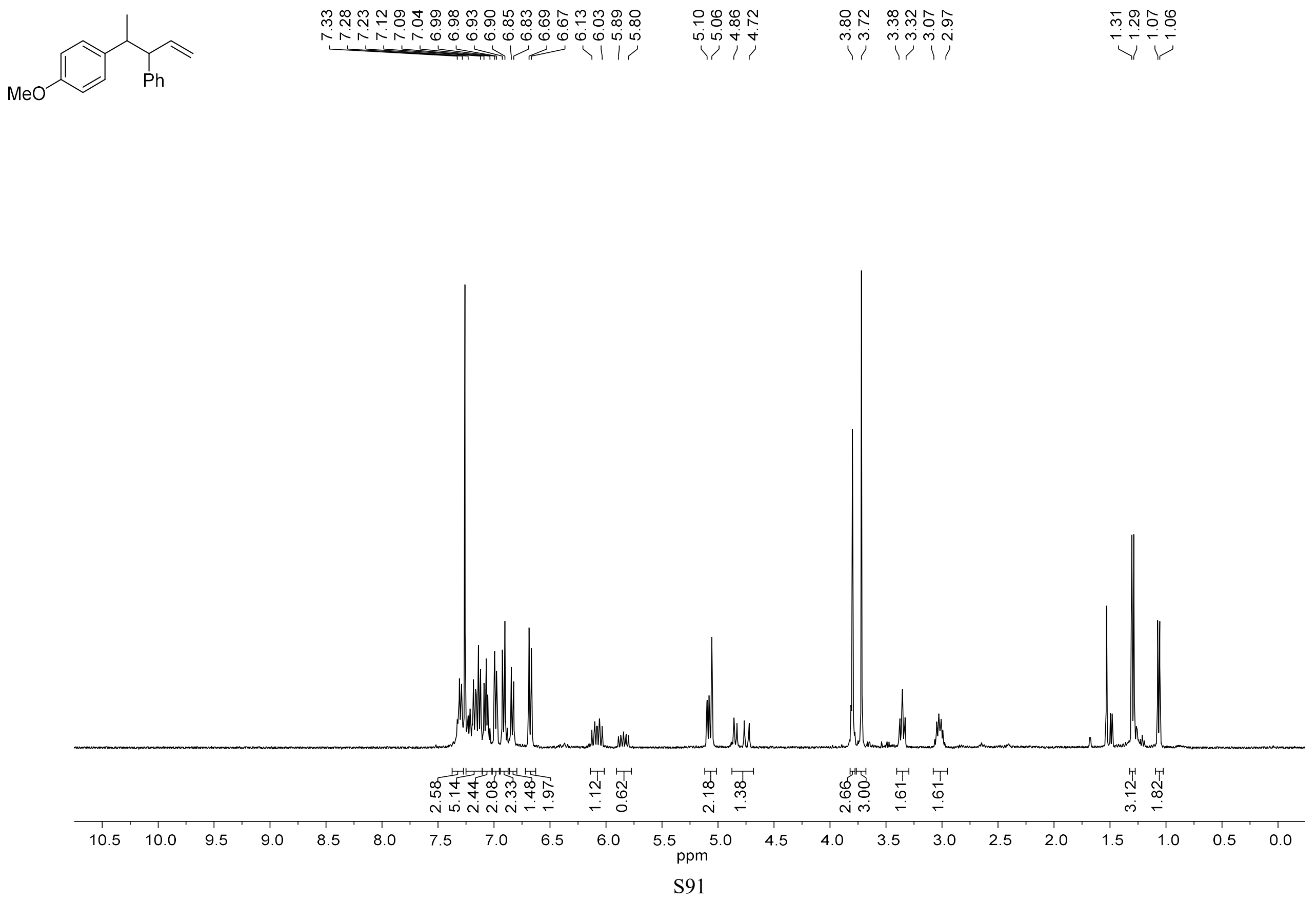
$38(64: 36 \mathrm{dr}){ }^{13} \mathrm{C}\left\{{ }^{1} \mathrm{H}\right\}$ NMR $\left(126 \mathrm{MHz}, \mathrm{CDCl}_{3}\right)$

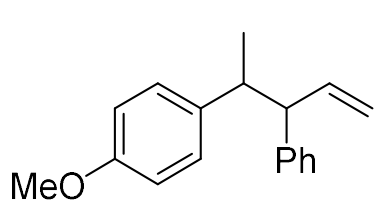

V|l

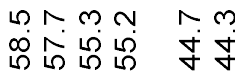

पi

용ํำ

hh

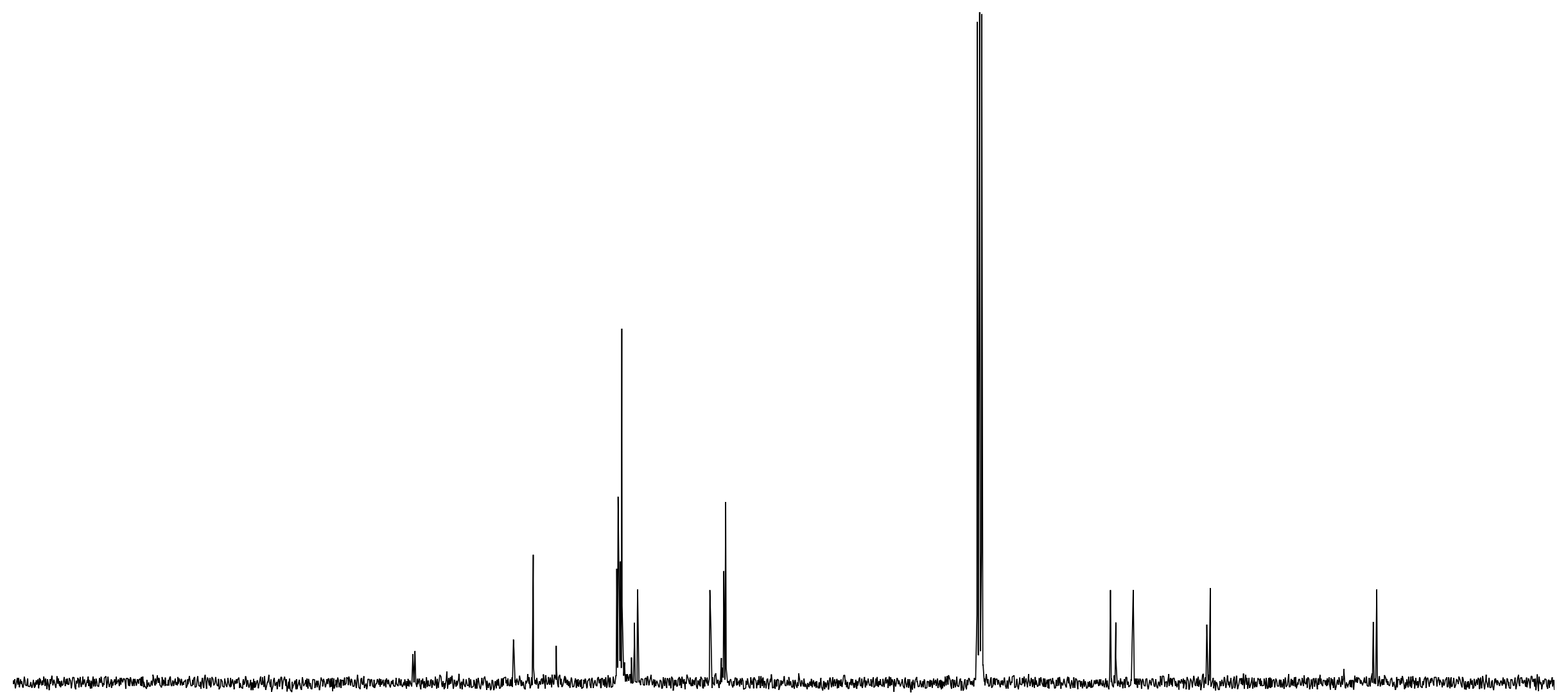

210

$190 \quad 180$

$170 \quad 160$

150

140

130

120

110

$90 \quad 80 \quad 70$

60

50

40

30

20 
S13 and S14 (87:13) ${ }^{1} \mathrm{H}$ NMR (400 MHz, $\left.\mathrm{CDCl}_{3}\right)$

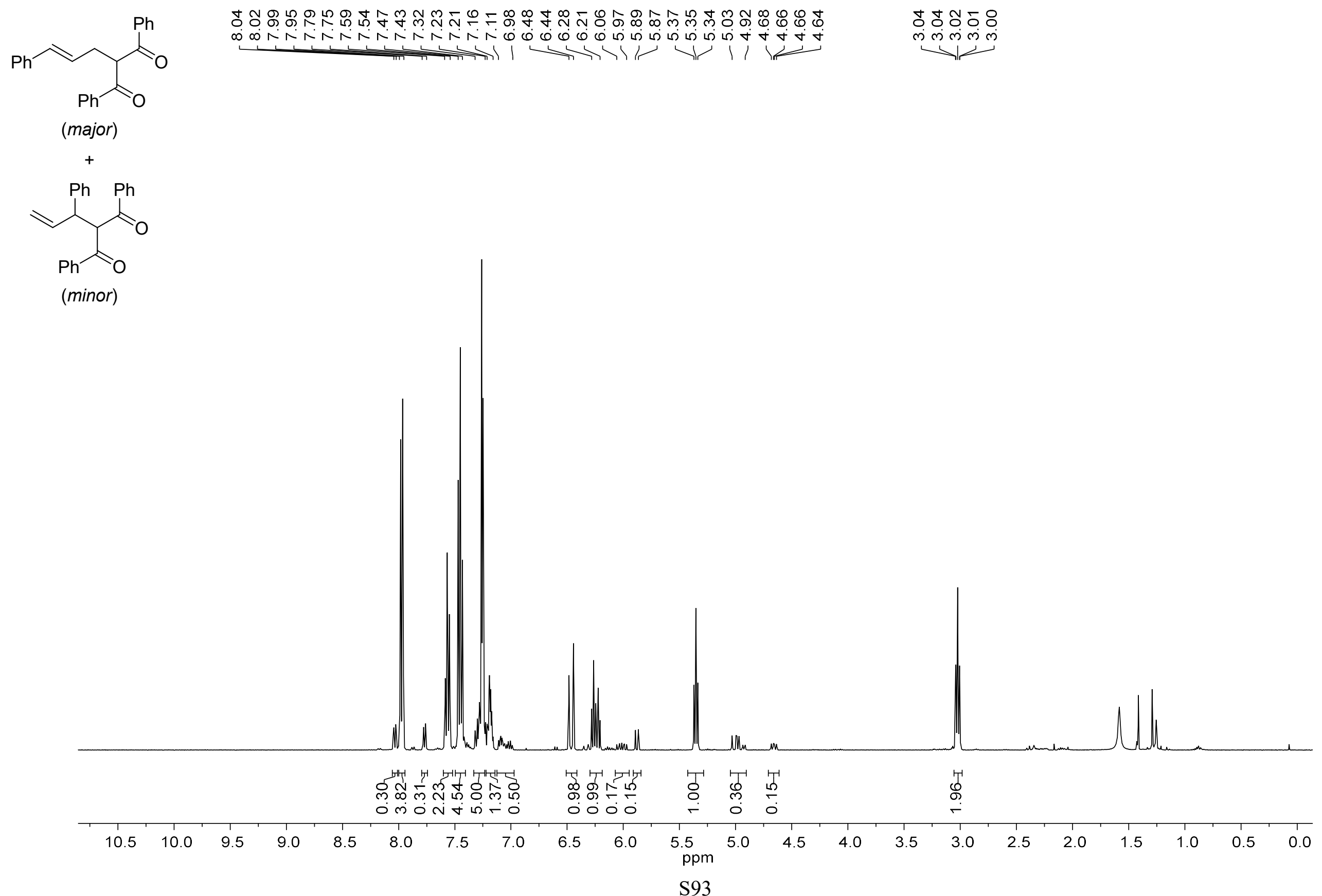




\section{S15 ${ }^{1} \mathrm{H}$ NMR $\left(300 \mathrm{MHz}, \mathrm{CDCl}_{3}\right)$}

ల్లై

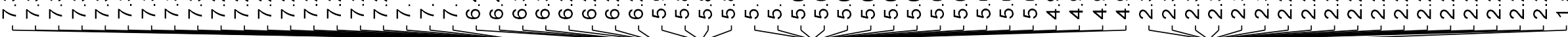<smiles>C=CCCC=Cc1ccccc1</smiles>

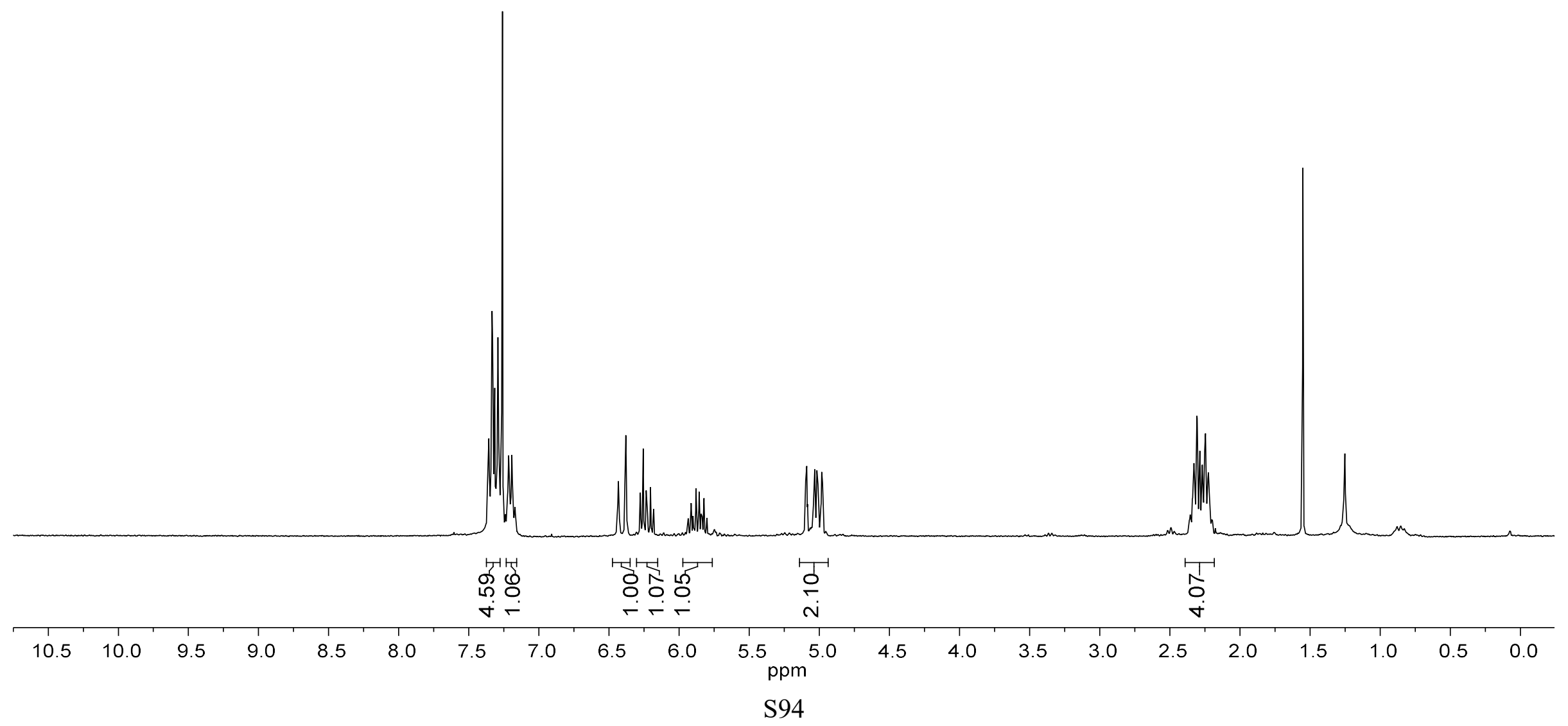




\section{References}

(1) Ang, H. T.; Rygus, J. P. G.; Hall, D. G. Two-component boronic acid catalysis for increased reactivity in challenging Friedel-Crafts alkylations with deactivated benzylic alcohols. Org. Biomol. Chem. 2019, 17, 6007-6014.

(2) Hsu, S.-F.; Plietker, B. Selective Transfer Hydrogenation and Hydrogenation of Ketones Using a Defined Monofunctional $\left(\mathrm{P}^{\wedge} \mathrm{N}(\mathrm{Bn})^{\wedge} \mathrm{N}(\mathrm{Bn})^{\wedge} \mathrm{P}\right)-\mathrm{RuII}$ Complex. Chem. Eur. J. 2014, 20, 4242-4245.

(3) Süsse, L.; Hermeke, J.; Oestreich, M. The Asymmetric Piers Hydrosilylation. J. Am. Chem. Soc. 2016, 138, 6940-6943.

(4) Barreiro, E.; Sanz-Vidal, A.; Tan, E.; Lau, S.-H.; Sheppard, T. D.; DíezGonzález, S. HBF4-Catalysed Nucleophilic Substitutions of Propargylic Alcohols. Eur. J. Org. Chem. 2015, 2015, 7544-7549.

(5) Bruneau-Voisine, A.; Wang, D.; Dorcet, V.; Roisnel, T.; Darcel, C.; Sortais, J.B. Transfer Hydrogenation of Carbonyl Derivatives Catalyzed by an Inexpensive PhosphineFree Manganese Precatalyst. Org. Lett. 2017, 19, 3656-3659.

(6) Nokami, T.; Yamane, Y.; Oshitani, S.; Kobayashi, J.-k.; Matsui, S.-i.; Nishihara, T.; Uno, H.; Hayase, S.; Itoh, T. The $\beta$-Silyl Effect on the Memory of Chirality in Friedel-Crafts Alkylation Using Chiral $\alpha$-Aryl Alcohols. Org. Lett. 2015, 17, 3182-3185.

(7) Łowicki, D.; Bezłada, A.; Mlynarski, J. Asymmetric Hydrosilylation of Ketones Catalyzed by Zinc Acetate with Hindered Pybox Ligands. Adv. Synth. Catal. 2014, $356,591-595$.

(8) Schulte, B.; Fröhlich, R.; Studer, A. Atroposelective radical aryl migration reactions from sulfur to carbon. Tetrahedron 2008, 64, 11852-11859.

(9) Garcia, K. J.; Gilbert, M. M.; Weix, D. J. Nickel-Catalyzed Addition of Aryl Bromides to Aldehydes To Form Hindered Secondary Alcohols. J. Am. Chem. Soc. 2019, $141,1823-1827$.

(10) Yamashita, Y.; Suzuki, H.; Sato, I.; Hirata, T.; Kobayashi, S. Catalytic DirectType Addition Reactions of Alkylarenes with Imines and Alkenes. Angew. Chem. Int. Ed. 
2018, 57, 6896-6900.

(11) Hayashi, K.; Tanimoto, H.; Zhang, H.; Morimoto, T.; Nishiyama, Y.; Kakiuchi, K. Efficient Synthesis of $\alpha, \beta$-Unsaturated Alkylimines Performed with Allyl Cations and Azides: Application to the Synthesis of an Ant Venom Alkaloid. Org. Lett. 2012, 14, 5728 5731.

(12) Zou, L.-H.; Priebbenow, D. L.; Wang, L.; Mottweiler, J.; Bolm, C. CopperCatalyzed Synthesis of $\alpha$-Thioaryl Carbonyl Compounds Through S $\square \mathrm{S}$ and $\mathrm{C} \square \mathrm{C}$ Bond Cleavage. Adv. Synth. Catal. 2013, 355, 2558-2563.

(13) Rao, H. S. P.; Muthanna, N. Variations in the Blaise Reaction: Conceptually New Synthesis of 3-Amino Enones and 1,3-Diketones. Eur. J. Org. Chem. 2015, 2015, 1525 1532.

(14) Betterley, N. M.; Surawatanawong, P.; Prabpai, S.; Kongsaeree, P.; Kuhakarn, C.; Pohmakotr, M.; Reutrakul, V. Electrophilic Difluoro(phenylthio)methylation: Generation, Stability, and Reactivity of $\alpha$-Fluorocarbocations. Org. Lett. 2013, 15, 5666-5669.

(15) Correia, C. A.; Li, C.-J. Catalytic alkylation of benzylic C-H bonds with 1,3dicarbonyl compounds utilizing oxygen as terminal oxidant. Tetrahedron Lett. 2010, 51, 1172-1175.

(16) Noji, M.; Konno, Y.; Ishii, K. Metal Triflate-Catalyzed Cationic Benzylation and Allylation of 1,3-Dicarbonyl Compounds. J. Org. Chem. 2007, 72, 5161-5167.

(17) Liu, P. N.; Dang, L.; Wang, Q. W.; Zhao, S. L.; Xia, F.; Ren, Y. J.; Gong, X. Q.; Chen, J. Q. Perchloric Acid Catalyzed Homogeneous and Heterogeneous Addition of $\beta$ Dicarbonyl Compounds to Alcohols and Alkenes and Investigation of the Mechanism. J. Org. Chem. 2010, 75, 5017-5030.

(18) Chatterjee, P. N.; Roy, S. Alkylation of 1,3-dicarbonyl compounds with benzylic and propargylic alcohols using Ir-Sn bimetallic catalyst: synthesis of fully decorated furans and pyrroles. Tetrahedron 2011, 67, 4569-4577.

(19) Sanz, R.; Miguel, D.; Martínez, A.; Álvarez-Gutiérrez, J. M.; Rodríguez, F. Brønsted Acid-Catalyzed Benzylation of 1,3-Dicarbonyl Derivatives. Org. Lett. 2007, 9 , 
2027-2030.

(20) Cheng, H.-G.; Feng, B.; Chen, L.-Y.; Guo, W.; Yu, X.-Y.; Lu, L.-Q.; Chen, J.R.; Xiao, W.-J. Rational design of sulfoxide-phosphine ligands for Pd-catalyzed enantioselective allylic alkylation reactions. Chem. Commun. 2014, 50, 2873-2875.

(21) Gao, W.-C.; Jiang, S.; Wang, R.-L.; Zhang, C. Iodine-mediated intramolecular amination of ketones: the synthesis of 2-acylindoles and 2-acylindolines by tuning $\mathrm{N}$ protecting groups. Chem. Commun. 2013, 49, 4890-4892.

(22) Zhu, Q.; Gentry, E. C.; Knowles, R. R. Catalytic Carbocation Generation Enabled by the Mesolytic Cleavage of Alkoxyamine Radical Cations. Angew. Chem. Int. Ed. 2016, 55, 9969-9973.

(23) Umeda, R.; Jikyo, T.; Toda, K.; Osaka, I.; Nishiyama, Y. Rhenium complexcatalyzed carbon-carbon formation of alcohols and organosilicon compounds. Tetrahedron Lett. 2018, 59, 1121-1124.

(24) Saito, T.; Nishimoto, Y.; Yasuda, M.; Baba, A. Direct Coupling Reaction between Alcohols and Silyl Compounds: Enhancement of Lewis Acidity of Me3SiBr Using InC13. J. Org. Chem. 2006, 71, 8516-8522.

(25) Bohan, P. T.; Toste, F. D. Well-Defined Chiral Gold(III) Complex Catalyzed Direct Enantioconvergent Kinetic Resolution of 1,5-Enynes. J. Am. Chem. Soc. 2017, 139, 11016-11019.

(26) Durand, A. C.; Brahmi, L.; Lahrech, M.; Hacini, S.; Santelli, M. Preparation of 4 - Arylcyclopentenes by Sequential Diallylation of Arylaldehydes and Ring - Closing Metathesis. Synth. Commun. 2005, 35, 1825-1833.

(27) Mahoney, S. J.; Lou, T.; Bondarenko, G.; Fillion, E. Carbon-Based Leaving Group in Substitution Reactions: Functionalization of sp3-Hybridized Quaternary and Tertiary Benzylic Carbon Centers. Org. Lett. 2012, 14, 3474-3477.

(28) Cao, H.; Jiang, H.; Feng, H.; Kwan, J. M. C.; Liu, X.; Wu, J. Photo-induced Decarboxylative Heck-Type Coupling of Unactivated Aliphatic Acids and Terminal Alkenes in the Absence of Sacrificial Hydrogen Acceptors. J. Am. Chem. Soc. 2018, 140, 16360- 
16367. 